Draft VERSION NOVEMBER 5, 2018

Preprint typeset using $\mathrm{LAT}_{\mathrm{E}} \mathrm{X}$ style emulateapj v. 5/2/11

\title{
A KINEMATIC STUDY OF THE ANDROMEDA DWARF SPHEROIDAL SYSTEM
}

\author{
Michelle L. M. Collins ${ }^{1,2}$, Scott C. Chapman ${ }^{2,3}$, R. Michael Rich ${ }^{4}$, Rodrigo A. Ibata ${ }^{5}$, Nicolas F. Martin ${ }^{5,1}$, \\ Michael J. Irwin ${ }^{2}$, Nicholas F. Bate ${ }^{6}$, Geraint F. Lewis ${ }^{6}$, Jorge Peñarrubia ${ }^{7,8}$, Nobuo Arimoto ${ }^{9,10}$, Caitlin M. \\ Casey $^{11}$, Annette M. N. Ferguson ${ }^{8}$, Andreas Koch ${ }^{12}$, Alan W. McConnachie ${ }^{13}$, Nial Tanvir ${ }^{14}$
}

Draft version November 5, 2018

\begin{abstract}
We present a homogeneous kinematic analysis of red giant branch stars within 18 of the 28 Andromeda dwarf spheroidal (dSph) galaxies, obtained using the Keck I LRIS and Keck II DEIMOS spectrographs. Based on their $g-i$ colors (taken with the CFHT MegaCam imager), physical positions on the sky, and radial velocities, we assign probabilities of $\mathrm{d}$ Sph membership to each observed star. Using this information, the velocity dispersions, central masses and central densities of the dark matter halos are calculated for these objects, and compared with the properties of the Milky Way $\mathrm{dSph}$ population. We also measure the average metallicity $([\mathrm{Fe} / \mathrm{H}])$ from the co-added spectra of member stars for each M31 dSph and find that they are consistent with the trend of decreasing $[\mathrm{Fe} / \mathrm{H}]$ with luminosity observed in the Milky Way population. We find that three of our studied M31 dSphs appear as significant outliers in terms of their central velocity dispersion, And XIX, XXI and XXV, all of which have large half-light radii $(\gtrsim 700 \mathrm{pc})$ and low velocity dispersions $\left(\sigma_{v}<5 \mathrm{~km} \mathrm{~s}^{-1}\right)$. In addition, And XXV has a mass-to-light ratio within its half-light radius of just $[M / L]_{\text {half }}=10.3_{-6.7}^{+7.0}$, making it consistent with a simple stellar system with no appreciable dark matter component within its $1 \sigma$ uncertainties. We suggest that the structure of the dark matter halos of these outliers have been significantly altered by tides.

Subject headings: dark matter — galaxies: dwarf — galaxies: fundamental parameters — galaxies: kinematics and dynamics — Local Group
\end{abstract}

\section{INTRODUCTION}

The underlying nature of the dark matter halos of dwarf spheroidal galaxies (dSphs) has garnered significant attention from the scientific community over the past decade. The goal of recent observational studies of these objects has been to make critical tests of structure formation scenarios, particularly focusing on the viability of the canonical $\Lambda$ CDM model. There is the long standing issue of the relative dearth of these faintest of galax-

\footnotetext{
${ }^{1}$ Max-Planck-Institut für Astronomie, Königstuhl 17, D69117 Heidelberg, Germany

${ }^{2}$ Institute of Astronomy,Madingley Rise, Cambridge, CB3 OHA , UK

${ }^{3}$ Dalhousie University Dept. of Physics and Atmospheric Science Coburg Road Halifax, B3H1A6, Canada

${ }^{4}$ Department of Physics and Astronomy, University of California, Los Angeles, CA 90095-1547

${ }^{5}$ Observatoire astronomique de Strasbourg, Universit de Strasbourg, CNRS, UMR 7550, 11 rue de lUniversit, F-67000 Strasbourg, France

${ }^{6}$ Sydney Institute for Astronomy, School of Physics, A28, University of Sydney, NSW 2006, Australia

${ }^{7}$ Ramón y Cajal Fellow, Instituto de Astrofísica de AndaluciaCSIC, Glorieta de la Astronomía s/n, 18008, Granada, Spain

8 Institute for Astronomy, University of Edinburgh, Royal Observatory, Blackford Hill, Edinburgh, EH9 3HJ, UK

9 Subaru Telescope, National Astronomical Observatory of Japan 650 North A'ohoku Place, Hilo, Hawaii 96720, U.S.A.

${ }_{10}$ Graduate University for Advanced Studies 2-21-1 Osawa, Mitaka, Tokyo 181-8588, Japan

${ }^{11}$ Institute for Astronomy, 2680 Woodlawn Drive Honolulu, HI 96822-1839 USA

12 Zentrum für Astronomie der Universität Heidelberg, Landessternwarte, Königstuhl 12, 69117 Heidelberg, Germany

${ }^{13}$ NRC Herzberg Institute of Astrophysics, 5071 West Saanich Road, British Columbia, Victoria V9E 2E7, Canada

14 Department of Physics \& Astronomy, University of Leicester, University Road, Leicester LE1 7RH, UK
}

ies observed surrounding nearby galaxies when compared with the number of dark matter subhalos produced in $N$-body simulations, which is referred to as the "missing satellite" problem (Klypin et al. 1999; Moore et al. 1999). The extent to which this mismatch is considered problematic has decreased over recent years as both theorists and observers have sought to reconcile the simulated and observable Universe. From a modelling point of view, one does not expect stars to be able to form within all dark matter subhalos seen in simulations, and at a certain mass limit $\left(V_{\max } \lesssim 15 \mathrm{~km} \mathrm{~s}^{-1}\right.$, see Peñarrubia et al. 2008a; Koposov et al. 2009), star formation is unable to proceed. Thus, there is a lower limit placed on galaxy formation. This mass limit is also tied to feedback processes that can remove the baryonic reservoirs required for star formation (e.g., Bullock et al. 2000; Somerville 2002; Kravtsov 2010; Bullock et al. 2010; Nickerson et al. 2011; Kazantzidis et al. 2011). This would imply that only the most massive subhalos seen in simulations are able to form and retain luminous populations. Observers have also attempted to quantify current survey completeness and radial selection effects to account for the number of satellites we are not currently able to detect (e.g., Koposov et al. 2008; Tollerud et al. 2008; Walsh et al. 2009). These studies suggest that there are of order a few hundred satellites within the Milky Way's virial radius that we have yet to detect.

The high dark matter dominance of dSph galaxies also singles them out as objects of interest. With total dynamic mass-to-light ratios of $[M / L] \sim 10-1000$ s and half-light radii of $r_{\text {half }} \sim 10-1000 \mathrm{pc}$, they are ideal systems with which to probe the inner density profiles of dark matter halos. Recent imaging and spec- 
troscopic observations of these objects within the Local Group have shown that, despite spanning approximately 5 decades in luminosity, the dSphs of the MW share a common mass scale and a universal density profile (Strigari et al. 2008; Walker et al. 2009b; Wolf et al. 2010). With a kinematic resolution of a few 10s of parsecs, these objects allow us to start addressing the question of whether the central regions of these halos follow cuspy density profiles as predicted by cosmological simulations (Navarro et al. 1997), or constant density cores, similar to what is observed in low surface brightness galaxies (Blais-Ouellette et al. 2001; de Blok \& Bosma 2002; de Blok et al. 2003; de Blok 2005; Swaters et al. 2003; Kassin et al. 2006; Spano et al. 2008). From studies of brighter dSphs, such as Sculptor and Fornax (Walker \& Peñarrubia 2011; Amorisco \& Evans 2012; Jardel \& Gebhardt 2012) it appears that their halo density profiles are also inconsistent with hosting central cusps. It is possible that these objects originally formed with cuspy density profiles, and that these have been subsequently modified by baryonic feedback. If this is truly the case, one should be able to observe cuspy profiles in the fainter dSph population (Zolotov et al. 2012) as these do not contain enough baryons to drive this change in the dark matter density profile. Perhaps the only way to gain further insight into this contentious issue is by measuring the kinematics for large numbers of stellar tracers within these objects and analysing them with detailed models that do not make $a b$ initio assumptions about the underlying density profiles, or the velocity anisotropy of both the dark matter and stars, such as those employed by Walker \& Peñarrubia (2011) and Jardel \& Gebhardt (2012).

To date, the majority of studies involving the detailed kinematics of dSphs have revolved largely around those belonging to the Milky Way, as these are nearby enough that we can measure the velocities of their member stars to a high degree of accuracy. However, there are currently only $\sim 25$ known MW dSphs, with luminosities ranging from $10^{2}-10^{7} \mathrm{~L}_{\odot}$. For the very faintest, some controversy remains as to whether they are massively dark-matter dominated (see e.g., Niederste-Ostholt et al. 2009; Simon et al. 2011), but almost all of them have been shown to be consistent with the universal mass profiles of Walker et al. (2009a) and Wolf et al. (2010). One notable exception to this is the Hercules object (Adén et al. 2009), which some have argued is currently undergoing significant tidal disruption (Martin \& Jin 2010). Andromeda represents the only other system for which comparable kinematic analyses can be performed. M31 now has $28 \mathrm{dSph}$ companions known, whose luminosities range from $10^{4}-10^{8} \mathrm{~L}_{\odot}$, the majority of which have been discovered by the CFHT Pan-Andromeda Archaeological Survey (PAndAS Martin et al. 2006; Ibata et al. 2007; Irwin et al. 2008; McConnachie et al. 2008; Martin et al. 2009; Richardson et al. 2011). The relatively brighter lower bound for the luminosities of M31 dSphs compared to the MW is a detection limit issue, rather than a sign of differing stellar populations (Martin et al. 2013 , in prep). It has been noted by a number of authors (e.g., McConnachie \& Irwin 2006b; Tollerud et al. 2012; McConnachie 2012) that for the brighter dSphs $\left(M_{V}<-8\right)$, those belonging to M31 are 2-3 times more extended in terms of their half-light $\left(r_{\text {half }}\right)$ and tidal $\left(r_{t}\right)$ radii compared with the MW. In these papers, the underlying cause of this discrepancy was not identified, but it has been argued that it could be an effect of environment, with the mass distribution of the host playing an important role. Subsequent work by Brasseur et al. (2011a), who included the fainter, non-classic M31 dSphs for the first time, showed that statistically, the relationship between size and luminosity for dSphs in the MW and Andromeda are actually largely consistent with one another, however there remain a number of significantly extended outliers within the Andromedean system (e.g., And II, And XIX, $r_{\text {half }} \sim 1.2 \mathrm{kpc}$ and $1.5 \mathrm{kpc}$ respectively), and the scatter in this relationship is large (up to an order of magnitude at a given luminosity, McConnachie|2012).

Working from the McConnachie \& Irwin (2006b) results, Peñarrubia et al. (2008a) modelled the expected velocity dispersions for the M31 dSphs, assuming that all dSph galaxies are embedded within similar mass dark matter halos. A robust prediction of their modeling was that, given the larger radial extents, the dSphs of M31 should be kinematically hotter than their MW counterparts by a factor of $\sim 2$. At the time of writing, they had only 2 measured velocity dispersions for the M31 dSphs, those of And II and And IX (Côté et al. 1999; Chapman et al. 2005). New studies of the kinematics of M31 dSphs (Collins et al.|2010, 2011; Kalirai et al.|2010; Tollerud et al. 2012; Chapman et al. 2012) have dramatically increased the number of systems with a measured velocity dispersion, and have shown that instead of being kinematically hotter, these systems are either very similar to, or in a number of cases (e.g., And II, And XII, And XIV, And XV and And XXII), colder than their MW counterparts. In particular, a significant recent kinematic study of 15 M31 dSph companions using the Keck II DEIMOS spectrograph by the Spectroscopic and Photometric Landscape of Andromeda's Stellar Halo (SPLASH, Tollerud et al. 2012) concluded that the M31 dSph system largely obeys very similar masssize-luminosity scalings as those of the MW. However, they also identified 3 outliers (And XIV, XV and XVI) that appear to possess much lower velocity dispersions, and hence maximum circular velocities, than would be expected for these systems. Such a result suggests that there are significant differences in the formation and/or evolution of the M31 and MW dSph systems.

To investigate this further, our group has been systematically surveying the known dSphs of M31 with the Keck I LRIS and Keck II DEIMOS spectrographs, and have obtained kinematic data for 18 of the 28 galaxies. In this paper, we present new spectroscopic analysis for the 11 dSphs, Andromeda (And) XVII, And XVIII, And XIX, And XX, And XXI, And XXIII, And XXIV, And XXV, And XXVI, the tidally disrupting And XXVII, And XXVIII and And XXX (Cassiopeia II) using an algorithm we have developed that implements a probabilistic method of determining membership for each galaxy. In addition we re-analyze the kinematics of $6 \mathrm{dSphs}$ that our group has previously observed (And V, VI, XI, XII, XIII, XXII) using this method with the aim of providing a homogeneous analysis of all dSphs observed by our group to date. We also provide the individual stellar velocities and properties for every star observed in our dSphs survey, allowing us to present a large catalog of 
stellar kinematics that will be of interest to those studying dSph systems and Milky Way-like galaxies, whether observationally or theoretically.

The outline of this paper is as follows. In $\S 2$ we discuss the relevant observations, data reduction techniques. In $\S 3$ we outline our algorithm for the classification of member stars. In $\S 4$ we present an analysis of our new kinematic datasets. In $\S 5$ we report on the masses and densities of the dark matter halos of the M31 dSphs, comparing them to those of the MW dSphs. In $\S 6$ we report on the metallicities, $[\mathrm{Fe} / \mathrm{H}]$, of the M31 dSphs as measured from the co-added spectra of their member stars. Finally, we conclude in $\S 7$.

\section{OBSERVATIONS}

\subsection{Photometry and target selection}

The PAndAS survey (McConnachie et al. 2009), conducted using the 3.6 metre Canada France Hawaii Telescope (CFHT), maps out the stellar density of the disc and halo regions of the M31-M33 system over a projected area of $\sim 350 \mathrm{deg}^{2}\left(\sim 55,000 \mathrm{kpc}^{2}\right)$, resolving individual stars to depths of $g=26.5$ and $i=25.5$ with a signal to noise ratio of 10 , making this survey the deepest, highest resolution, contiguous map of the majority of the extended stellar halo of an $\mathrm{L}_{*}$ galaxy to date. Each of the 411 fields in this survey $\left(0.96 \times 0.94 \mathrm{deg}^{2}\right)$ has been observed for at least 1350s in both MegaCam $g$ and $i$ filters, in $<0.8^{\prime \prime}$ seeing. This survey was initiated following two precursor surveys of the M31 system, the first of which surveyed the central $\sim 40 \mathrm{deg}^{2}$ conducted with the 2.5 metre Isaac Newton Telescope (Ferguson et al. 2002; Irwin et al. 2005), and revealed a wealth of substructure in the Andromeda stellar halo, including the giant southern stream (Ibata et al. 2001). To better understand this feature, and to probe deeper into the M31 (and M33) stellar halo, a survey of the south west quadrant of the M31 halo was initiated using the CFHT (Ibata et al. 2007), and revealed yet more substructure, including the arc like stream $\mathrm{Cp}$ and $\mathrm{Cr}$ (Chapman et al. 2008) and a number of dwarf spheroidal satellites (Martin et al. 2006). This CFHT survey was then extended into the full PAndAS project. For details of the processing and reduction of these data, see Richardson et al. (2011). This survey has introduced us to a wealth of stellar substructure, debris and globular clusters within the Andromeda-Triangulum system. In addition, it has led to the discovery of 17 dSphs. These objects were detected in the PAndAS survey maps as over-densities in matched-filter surface density maps of metal poor red giant branch (RGB) stars and were presented in Martin et al. (2006); Ibata et al. (2007); Irwin et al. (2008); McConnachie et al. (2008); Martin et al. (2009) and Richardson et al. (2011). We briefly summarise the photometric properties of all dSphs discussed within this paper in Table 1.

For the majority of these objects, the PAndAS dataset formed the basis for our spectroscopic target selection. Using the color selection boxes presented in McConnachie et al. (2008); Martin et al. (2009) and Richardson et al. (2011), we isolated the RGBs of each dSph, then prioritised each star on this sequence depending on their color, $i$-band magnitude, and distance from the centre of the dSph. Stars lying directly on the RGB, with $20.3<i_{0}<22.5$ and distance, $d<4 r_{\text {half }}$ (where $r_{\text {half }}$ is the half-light radius, measured on the semi-major axis of the dSph) were highly prioritised (priority A), followed by stars on the RGB within the same distance from the centre with $22.5<i_{0}<23.5$ (priority $\mathrm{B}$ ). The remainder of the mask was then filled with stars in the field with $20.3<i_{0}<23.5$ and $0.5<g-i<4$ (priority C). In general, it is the brighter, higher priority A stars that ultimately show the highest probability of membership. We also use the PAndAS photometry to help us determine membership of the dSph (discussed in $\S 3$ ).

And XXVIII is the one dSph in our sample that is not covered by the PAndAS survey. For this object, target selection followed an identical methodology tot hat detailed above, but used photometry from the 8th data release of the Sloan Digital Sky Survey (SDSS-III). Details of the observations and analysis of this photometry can be found in Slater et al. (2011).

\subsection{Keck Spectroscopic Observations}

The DEep-Imaging Multi-Object Spectrograph (DEIMOS), situated on the Nasmyth focus of the Keck II telescope is an ideal instrument for obtaining medium resolution ( $\mathrm{R} \sim 1.4 \stackrel{\circ}{\mathrm{A}}$ ) spectra of multiple, faint stellar targets in the M31 dSphs. The data for the dSphs within this work were taken between Sept 2004 and Sept 2012 in photometric conditions, with typical seeing between $0.5-1^{\prime \prime}$. Our chosen instrumental setting covered the observed wavelength range from 5600-9800 $\stackrel{\circ}{\mathrm{A}}$ and employed exposure times of $3 \times 20$ minute integrations. The majority of observations employed the 1200 line $/ \mathrm{mm}$ grating, although for $4 \mathrm{dSphs}$ (And XI, XII, XIII and XXIV) the lower resolution 600 line/mm grating ( $R \sim 3.8 \AA$ A FWHM) was used. The spectra from both setups typically possess signal-to-noise $(\mathrm{S}: \mathrm{N})$ ratios of $>3 \stackrel{\circ}{\mathrm{A}}^{-1}$ for our bright targets $(i \lesssim 22.0)$, though some of our fainter targets fall below this level. Information regarding the spectroscopic setup and observations for each dSph are displayed in Table 2 .

The resulting science spectra are reduced using a custom built pipeline, as described in Ibata et al. (2011). Briefly, the pipeline identifies and removes cosmic rays, corrects for scattered light, performs flat-fielding to correct for pixel-to-pixel variations, corrects for illumination, slit function and fringing, wavelength calibrates each pixel using arc-lamp exposures, performs a 2dimensional sky subtraction, and finally extracts each spectra - without resampling - in a small spatial region around the target. This results in a large set of pixels for each target, each of which carries a flux and wavelength (with associated uncertainties) plus the value of the target spatial profile at that pixel. We then derive velocities for all our stars with a Bayesian approach, using the Ca II Triplet absorption feature. Located at rest wavelengths of 8498,8542 and $8662 \stackrel{\circ}{\mathrm{A}}$, these strong features are ideal for determining the velocities of our observed stars. We determine the velocities by using an Markov Chain Monte Carlo procedure where a template Ca II spectrum was cross-correlated with the non-resampled data, generating a most-likely velocity for each star, and a likely uncertainty based on the posterior distribution that in- 
TABLE 1

Details OF THE STRUCTURAL PROPERTIES FOR EACH DWARF, AS DERIVED FROM CFHT PHOTOMETRY BY IBATA ET AL. (2007); LETARTE ET AL. (2009): IRWIN ET AL. (2008); MCCONNACHIE ET AL. (2008); MARTIN ET AL. (2009); Collins ET AL. (2010, 2011); BRASSEUR ET AL. (2011A); RICHARDSON ET AL. (2011) AND MCCONNACHIE (2012), UPDATED FOR DISTANCES PRESENTED IN Conn ET AL. (2012). The Photometry For And VI Is Derived From Subaru SuprimeCam Photometry (COllins ET AL. 2011), and the VAlues For AND XXVIII COME FROM SDSS PHOTOMETRY (SLATER ET AL. 2011).

\begin{tabular}{lccccc}
\hline \hline \multicolumn{1}{c}{ Property } & $\alpha_{0, J 2000}$ & $\delta_{0, J 2000}$ & $M_{V}$ & $r_{\text {half }}(\mathrm{pc})$ & $D(\mathrm{kpc})$ \\
\hline And V & $01: 10: 17.1$ & $+47: 37: 41.0$ & -10.2 & $302 \pm 44$ & $742_{-22}^{+21}$ \\
And VI & $23: 51: 39.0$ & $+24: 35: 42.0$ & -10.6 & $524 \pm 49$ & $783 \pm 28$ \\
And XI & $00: 46: 20.0$ & $+33: 48: 05.0$ & -6.9 & $158_{-23}^{+9}$ & $763_{-106}^{+29}$ \\
And XII & $00: 47: 27.0$ & $+34: 22: 29.0$ & -6.4 & $324_{-72}^{+56}$ & $928_{-136}^{+40}$ \\
And XIII & $00: 51: 51.0$ & $+33: 00: 16.0$ & -6.7 & $172_{-39}^{+34}$ & $760_{-154}^{+126}$ \\
And XVII & $00: 37: 07.0$ & $+44: 19: 20.0$ & -8.5 & $262_{-46}^{+53}$ & $727_{-25}^{+39}$ \\
And XVIII & $00: 02: 14.5$ & $+45: 05: 20.0$ & -9.7 & $325 \pm 24$ & $1214_{-43}^{+40}$ \\
And XIX & $00: 19: 32.1$ & $+35: 02: 37.1$ & -9.6 & $1481_{-268}^{+62}$ & $821_{-148}^{+32}$ \\
And XX & $00: 07: 30.7$ & $+35: 07: 56.4$ & -6.3 & $114_{-12}^{+31}$ & $741_{-52}^{+42}$ \\
And XXI & $23: 54: 47.7$ & $+42: 28: 15.0$ & -9.8 & $842 \pm 77$ & $827_{-25}^{+23}$ \\
And XXII & $01: 27: 40.0$ & $+28: 05: 25.0$ & -6.5 & $252_{-47}^{+28}$ & $920_{-139}^{+32}$ \\
And XXIII & $01: 29: 21.8$ & $+38: 43: 08.0$ & -10.2 & $1001_{-52}^{+53}$ & $748_{-21}^{+31}$ \\
And XXIV & $01: 18: 30.0$ & $+46: 21: 58.0$ & -7.6 & $548_{-37}^{+31}$ & $898_{-42}^{+28}$ \\
And XXV & $00: 30: 08.9$ & $+46: 51: 07.0$ & -9.7 & $642_{-74}^{+47}$ & $736_{-69}^{+23}$ \\
And XXVI & $00: 23: 45.6$ & $+47: 54: 58.0$ & -7.1 & $219_{-52}^{+67}$ & $754_{-164}^{+218}$ \\
And XXVII & $00: 37: 27.2$ & $+45: 23: 13.0$ & -7.9 & $657_{-271}^{+112}$ & $1255_{-474}^{+42}$ \\
And XXVIII & $22: 32: 41.2$ & $+31: 12: 51.2$ & -8.5 & $210_{-50}^{+60}$ & $650_{-80}^{+150}$ \\
And XXX (Cass II) & $00: 36: 34.9$ & $+49: 38: 48.0$ & -8.0 & $267_{-36}^{+23}$ & $681_{-78}^{+32}$ \\
& & & & & \\
\hline
\end{tabular}

corporates all the uncertainties for each pixel. Typically our velocity uncertainties lie in the range of $5-15 \mathrm{~km} \mathrm{~s}^{-1}$. Finally, we also correct these velocities to the heliocentric frame.

\subsubsection{Telluric velocity corrections}

With slit-spectroscopy, systematic velocity errors can be introduced if stars are not well aligned within the centre of their slits. Such misalignments can result from astrometric uncertainties or a slight offset in the position angle of the mask on the sky. For our astrometry, we take the positions of stars from PAndAS photometry, which have an internal accuracy of $\sim 0.1^{\prime \prime}$ and a global accuracy of $\sim 0 .^{\prime \prime} 25$ (Ségall et al. 2007). This can translate to velocity uncertainties of up to $\sim 15 \mathrm{~km} \mathrm{~s}^{-1}$ for our DEIMOS setup. In previous studies, authors have tried to correct for this effect by cross correlating their observed spectra with telluric absorption features (e.g., Sohn et al. 2007; Simon \& Geha 2007; Kalirai et al. 2010; Collins et al. 2010; Tollerud et al. 2012). These atmospheric absorption lines are superimposed on each science spectrum, and should always be observed at their rest-frame wavelengths. Thus, if one is able to determine the offset of these features, shifts caused by misalignment of the science star within the slit can be corrected for, and this can be applied on a slit by slit basis. The strongest of these features is the Fraunhofer A-band, located between 7595-7630 А. An example of this feature is shown in the left panel of Fig. 11.

While we believe this correction is robust in the high S:N regime, we argue against applying this correction in studies of Andromedean satellites, where $\mathrm{S}: \mathrm{N}$ is often quite low (typically less than 8-10 $\AA^{-1}$ ) for 1 hour observations of faint $(i \gtrsim 21)$ RGB stars. We find that when we compute this offset for all stars within our sample, those with high S:N tend to cluster within a few $\mathrm{kms}^{-1}$ of the average telluric correction found for the mask. As the S:N decreases below about $10 \stackrel{\circ}{\mathrm{A}}^{-1}$, the scatter about this mean value increases dramatically, as do the uncertainties computed for each individual correction. This is because the telluric feature is a single, very broad and asymmetric feature. It is therefore easy in the noisy regime for the cross correlation routine to misalign the template and science spectrum whilst still producing a high confidence cross-correlation maximum. We show this effect explicitly in the right hand panel of Fig. 11 where we plot the deviation of the telluric correction for every star within our sample from the average correction determined for the spectroscopic mask it was observed with $\left.\left(v_{t e l}-<v_{t e l}\right\rangle\right)$ as a function of S:N. The cyan points represent the individual data, and the large black points represent the median of all points within $1 \AA$ bins in $\mathrm{S}: \mathrm{N}$. The error bars represent the dispersion within each bin. It is plainly seen that the median value for each bin is consistent with zero (i.e., the median), and that the dispersion increases with decreasing $\mathrm{S}: \mathrm{N}$. If we were to apply these velocity corrections to all our stars, it is probable that we would merely increase the velocity uncertainties rather than reducing them.

For this reason, we take a different approach. Using solely the telluric velocity corrections of stars from each observed mask whose spectra have $\mathrm{S}: \mathrm{N}>7$, we measure (a) the average telluric correction for the mask and (b) the evolution of the telluric correction as a function of 
TABLE 2

DETAILS OF SPECTROSCOPIC OBSERVATIONS

\begin{tabular}{|c|c|c|c|c|c|c|c|c|c|c|}
\hline Object & Date & Instrument & Grating & Res. & $\alpha_{0, J 2000}$ & $\delta_{0, J 2000}$ & P.A. & Exp. (s) & $N_{\text {Targ }}$ & $N_{M e m}$ \\
\hline And $\mathrm{V}$ & 16 Aug 2009 & LRIS & $831 / 8200$ & $3.0 \AA$ & $01: 10: 18.21$ & $+47: 37: 53.3$ & $0^{\circ}$ & 3600 & 50 & 15 \\
\hline And VI & 17-19 Sept 2009 & DEIMOS & 1200 & $1.3 \AA$ & $23: 51: 51.49$ & $+24: 34: 57.0$ & $0^{\circ}$ & 5400 & 113 & 43 \\
\hline And XI & 23 Sept 2006 & DEIMOS & 600 & $3.5 \AA$ & $00: 46: 28.08$ & $+33: 46: 28.8$ & $0^{\circ}$ & 3600 & 33 & 5 \\
\hline And XII & 21-23 Sept 2006 & DEIMOS & 600 & $3.5 \stackrel{\circ}{\mathrm{A}}$ & $00: 47: 32.89$ & $+34: 22: 28.6$ & $0^{\circ}$ & 3600 & 49 & 8 \\
\hline And XIII & 23 Sept 2006 & DEIMOS & 600 & $3.5 \AA$ & $00: 52: 00.22$ & $+32: 59: 16.2$ & $0^{\circ}$ & 3600 & 46 & 4 \\
\hline And XVII & 26 Sept 2011 & DEIMOS & 1200 & $1.3 \AA$ & $00: 37: 51.09$ & $+44: 17: 51.9$ & $280^{\circ}$ & 3600 & 149 & 8 \\
\hline And XVIII & 9 Sept 2010 & DEIMOS & 1200 & $1.3 \mathrm{~A}$ & 00:02:14.50 & $+45: 05: 20.0$ & $90^{\circ}$ & 3600 & 73 & 4 \\
\hline And XIXa & 26 Sept 2011 & DEIMOS & 600 & $3.5 \AA$ & $00: 19: 45.04$ & $+35: 05: 28.8$ & $270^{\circ}$ & 3600 & 107 & 15 \\
\hline And XIXb & 26 Sept 2011 & DEIMOS & 1200 & $1.3 \AA$ & $00: 19: 30.88$ & $+35: 07: 34.1$ & $0^{\circ}$ & 3600 & 108 & 9 \\
\hline And XX & 9 Sept 2010 & DEIMOS & 1200 & $1.3 \AA$ & 00:07:30.69 & $+35: 08: 02.4$ & $90^{\circ}$ & 3600 & 85 & 4 \\
\hline And XXI & 26 Sept 2011 & DEIMOS & 1200 & $1.3 \AA$ & $23: 54: 47.70$ & $+42: 28: 33.6$ & $180^{\circ}$ & 3600 & 157 & 32 \\
\hline And XXIIa & 23 Sept 2009 & DEIMOS & 1200 & $1.3 \AA$ & $01: 27: 52.37$ & $+28: 05: 22.3$ & $90^{\circ}$ & 1200 & 93 & 4 \\
\hline And XXIIb & 10 Sept 2010 & DEIMOS & 1200 & $1.3 \AA$ & $01: 27: 52.37$ & $+28: 05: 22.3$ & $0^{\circ}$ & 3600 & 73 & 6 \\
\hline And XXIIIa & 9 Sept 2010 & DEIMOS & 1200 & $1.3 \AA$ & $01: 29: 18.18$ & $+38: 43: 50.4$ & $315^{\circ}$ & 3600 & 196 & 24 \\
\hline And XXIIIb & 10 Sept 2010 & DEIMOS & 1200 & $1.3 \AA$ & $01: 29: 21.87$ & $+38: 44: 58.7$ & $245^{\circ}$ & 3600 & 189 & 18 \\
\hline And XXIVa & 9 Sept 2010 & DEIMOS & 1200 & $1.3 \AA$ & 01:18:32.90 & $+46: 22: 50.0$ & $30^{\circ}$ & 3600 & 192 & 1 \\
\hline And XXIVb & 31 May 2011 & DEIMOS & 600 & $3.5 \stackrel{\circ}{\AA}$ & $01: 18: 32.90$ & $+46: 22: 50.0$ & $0^{\circ}$ & 2700 & 115 & 11 \\
\hline And XXV & 10 Sept 2010 & DEIMOS & 1200 & $1.3 \AA$ & $00: 30: 01.88$ & $+46: 50: 31.0$ & $90^{\circ}$ & 3600 & 183 & 26 \\
\hline And XXVI & 10 Sept 2010 & DEIMOS & 1200 & $1.3 \AA$ & $00: 23: 41.42$ & $+47: 54: 56.8$ & $90^{\circ}$ & 3600 & 179 & 6 \\
\hline And XXVII & 10 Sept 2010 & DEIMOS & 1200 & $1.3 \AA$ & $00: 37: 31.93$ & $+45: 23: 55.4$ & $45^{\circ}$ & 3600 & 131 & 8 \\
\hline And XXVIII & 21 Sept 2012 & DEIMOS & 1200 & $1.3 \AA$ & $22: 32: 32.71$ & $+31: 13: 13.2$ & $140^{\circ}$ & 3600 & 102 & 17 \\
\hline Cass II & 28 Sept 2011 & DEIMOS & 1200 & $1.3 \AA$ & $00: 37: 00.84$ & $+49: 39: 12.0$ & $270^{\circ}$ & 3600 & 156 & 8 \\
\hline
\end{tabular}
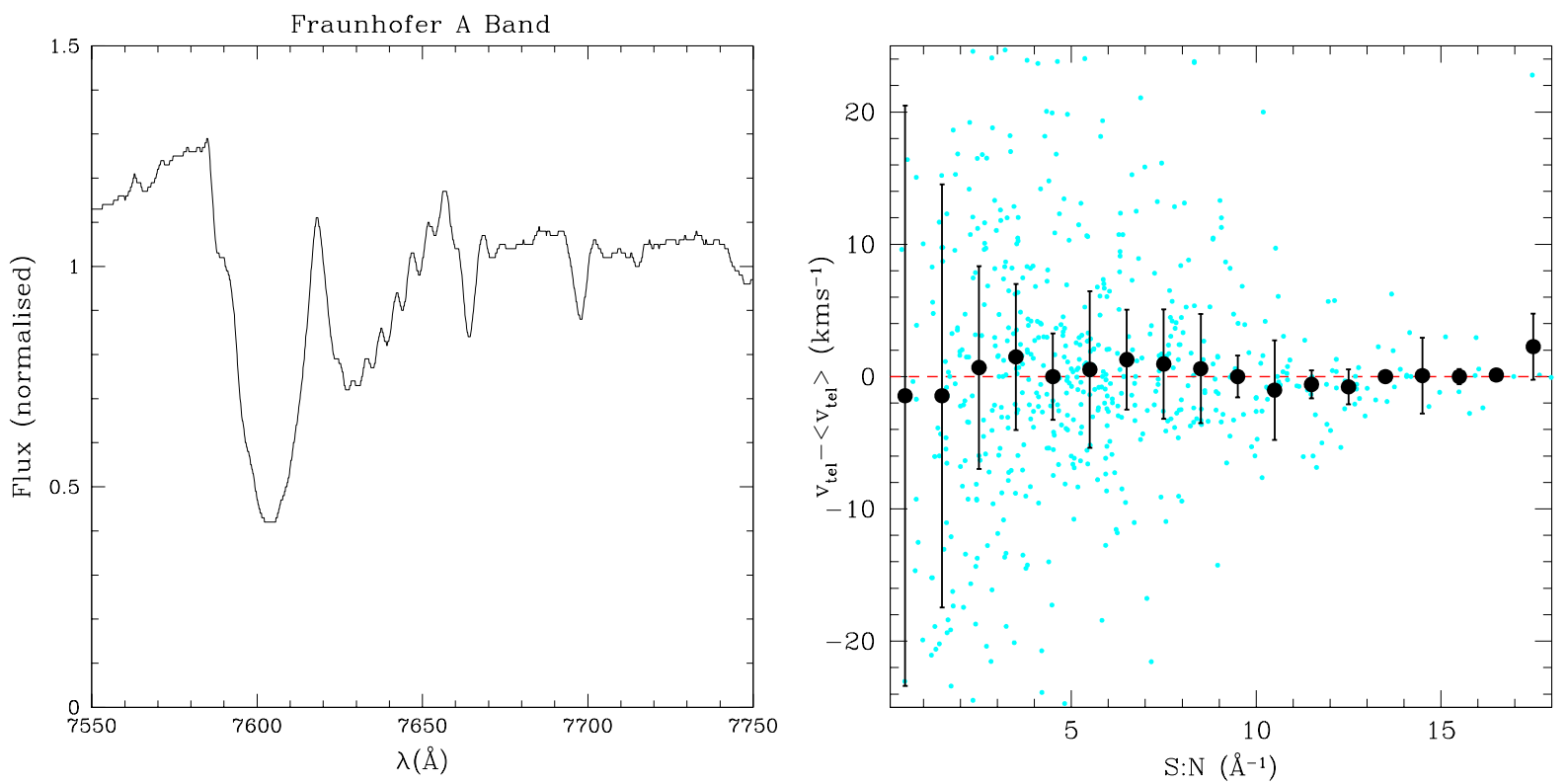

FIG. 1.- Left: Keck II DEIMOS spectrum of a bright foreground star, centered on the region of the strong telluric absorption feature, the Fraunhofer A band. Right: The deviation of the telluric correction for every observed star within our sample from the average correction determined for the spectroscopic mask $\left(v_{t e l}-\left\langle v_{t e l}\right\rangle\right)$ as a function of S:N. Cyan points represent the individual data, and the large black points represent the median of all points within $1 \AA$ bins in S:N. Error bars show the standard deviation within the bin. The median value for each bin is consistent with zero (i.e., the median), and the dispersion increases with decreasing S:N, arguing against using this correction in the low $\mathrm{S}: \mathrm{N}$ regime. 
mask position. In this way, we can track any gradient in our measurements that could be caused by e.g., rotational offsets in our mask. In general, we find these corrections to be slight. The average measured offset across all our masks is $3.8 \mathrm{~km} \mathrm{~s}^{-1}$ (ranging from between $-3.4 \mathrm{~km} \mathrm{~s}^{-1}$ and $+10.6 \mathrm{~km} \mathrm{~s}^{-1}$ ). The measured gradients are very slight, resulting in an average end-to-end mask difference of $2.6 \mathrm{~km} \mathrm{~s}^{-1}$, with a range of $0.1-7.2 \mathrm{~km} \mathrm{~s}^{-1}$, typically within our measured velocity uncertainties.

\section{A PROBABILISTIC DETERMINATION OF MEMBERSHIP}

Determining membership for Andromedean dwarf spheroidals is notoriously difficult in the best of cases. We only possess information about the velocity, CMD position, distance from the centre of the dSph and spectroscopic metallicity (although this carries large uncertainties of $>0.3$ dex for individual stars). Depending on the systemic velocity of the $\mathrm{dSph}$, we must try to use these properties to distinguish the likely members from either Milky Way halo K-dwarfs $\left(v_{\text {hel }} \gtrsim-150 \mathrm{kms}^{-1}\right)$ or M31 halo giants $\left(v_{\text {halo }} \approx-300 \mathrm{kms}^{-1}, \sigma_{v, \text { halo }} \approx\right.$ $90 \mathrm{~km} \mathrm{~s}^{-1}$, Chapman et al. 2006). In the case of Galactic contamination, our spectra also cover the region of the Na I doublets $(\sim 8100 \AA$ A). As this feature is dependent on the stellar surface gravity, it is typically stronger in dwarf stars than in giants. However, there is a significant overlap between the two, especially in the CMD color region of interest for Andromedean RGB stars. In the past, groups have focused on making hard cuts on likely members in an attempt to weed out likely contaminants (e.g., based on their distance from the centre of the galaxy or their velocity, Chapman et al. 2005; Collins et al. 2010, 2011; Kalirai et al. 2010), but such 'by eye' techniques are not particularly robust. Tollerud et al. (2012) recently presented an analysis of a number of M31 dSphs where they used a more statistical method to ascertain likely membership, using the distance from the centre of, and position in the CMD of stars targeted within their DEIMOS masks. Here we employ a similar technique that will assess the probability of stars being members of a dSph based on (i) their position on the CMD; (ii) their distance from the centre of the $\mathrm{dSph}$ and, in addition; (iii) their position in velocity space, giving the likelihood of membership as:

$$
P_{i} \propto P_{C M D} \times P_{\text {dist }} \times P_{\text {vel }}
$$

Below, we fully outline our method, and implement a series of tests to check it can robustly recover the kinematics of the M31 dSphs.

\subsection{Probability based on $C M D$ position, $P_{C M D}$}

For the first term, $P_{C M D}$, we are interested in where a given star observed in our mask falls with respect to the RGB of the observed dSph. Tollerud et al. (2012) use the distance of a given star from a fiducial isochrone fit to the dwarf photometry to measure this probability. Here, we determine this value from the data itself, rather than using isochrones. Using the PAndAS CFHT photometry, we construct a normalised Hess diagram for the central region (i.e., within $2 \times r_{\text {half }}$ ) of the $\mathrm{dSph}$, and one of a surrounding 'field' comparison region. By combining these two Hess diagrams, we can then map both the color distribution of the $\mathrm{dSph}$ and that of our contaminating populations. We use both directly as probability maps, where the densest region would have a value of 1 .

So we are not dominated by shot noise of sparsely populated regions of the CMD, we use only the region of the RGB. We do this by assigning a generous bounding box around the RGB as seen in the left hand panel of Fig. 2 where we display the PAndAS CMD for the well populated And XXI RGB. We have zoomed in on the region for which DEIMOS observations with reliable velocities can be obtained, e.g., $i<23.5$. The bounding box is shown with red dashed lines. Anything that falls outside this region is therefore assigned a probability of $P_{C M D}=0$. The resulting probability map for And XXI is shown in the right hand panel. Red points represent all DEIMOS stars that have $P_{C M D}>10^{-6}$, while the blue stars show stars from the DEIMOS mask that are far removed from the And XXI RGB, and thus not considered to be members.

\subsection{Probability based on distance position, $P_{\text {dist }}$}

The second term in our probability function, $P_{\text {dist }}$ can be easily determined from the known radial profile of the dSphs. The half-light radii of all these objects are known and can be found in McConnachie \& Irwin (2006a); Zucker et al. (2004, 2007); McConnachie et al. (2008); Martin et al. (2009); Collins et al. (2010, 2011); Richardson et al. (2011). We also know that their density profiles are well represented by a Plummer profile with a scale radius of $r_{p} \equiv r_{\text {half }}$. Therefore, we can define the probability function as a normalised Plummer profile (Plummer 1911), i.e.,:

$$
P_{\text {dist }}=\frac{1}{\pi r_{p}^{2}\left[1+\left(r / r_{p}\right)^{2}\right]^{2}}
$$

The above equation assumes that the systems we are studying are perfectly spherical. While the majority of these systems are not significantly elliptical, it is important to consider the effect of any observed deviations from sphericity. We therefore modify $r_{p}$ based on a given stars angular position with respect to the dwarfs major axis, $\theta_{i}$, such that:

$$
r_{p}=\frac{r_{\text {half }}(1-\epsilon)}{1+\epsilon \cos \theta_{i}}
$$

where $\epsilon$ is the measured ellipticity of the dSph as taken from McConnachie (2012).

\subsection{Probability based on velocity, $P_{\text {vel }}$}

The final term, $P_{v e l}$, contains information about the likelihood of a given star belonging to a kinematic substructure that is not well described by the velocity profiles of either the MW halo dwarfs or the Andromeda halo giants, both of which are determined empirically from our DEIMOS database of $>20000$ stars. From an analysis of these stars (selecting out any obvious substructures and the Andromedean disc) we find that the M31 halo is well approximated by a single Gaussian with a systemic velocity of $v_{r, \text { halo }}=-308.8 \mathrm{~km} \mathrm{~s}^{-1}$ and $\sigma_{v, \text { halo }}=96.3 \mathrm{~km} \mathrm{~s}^{-1}$, giving a probability density function for a given star with a velocity $v_{i}$ and velocity uncertainty of $v_{e r r, i}$ of: 

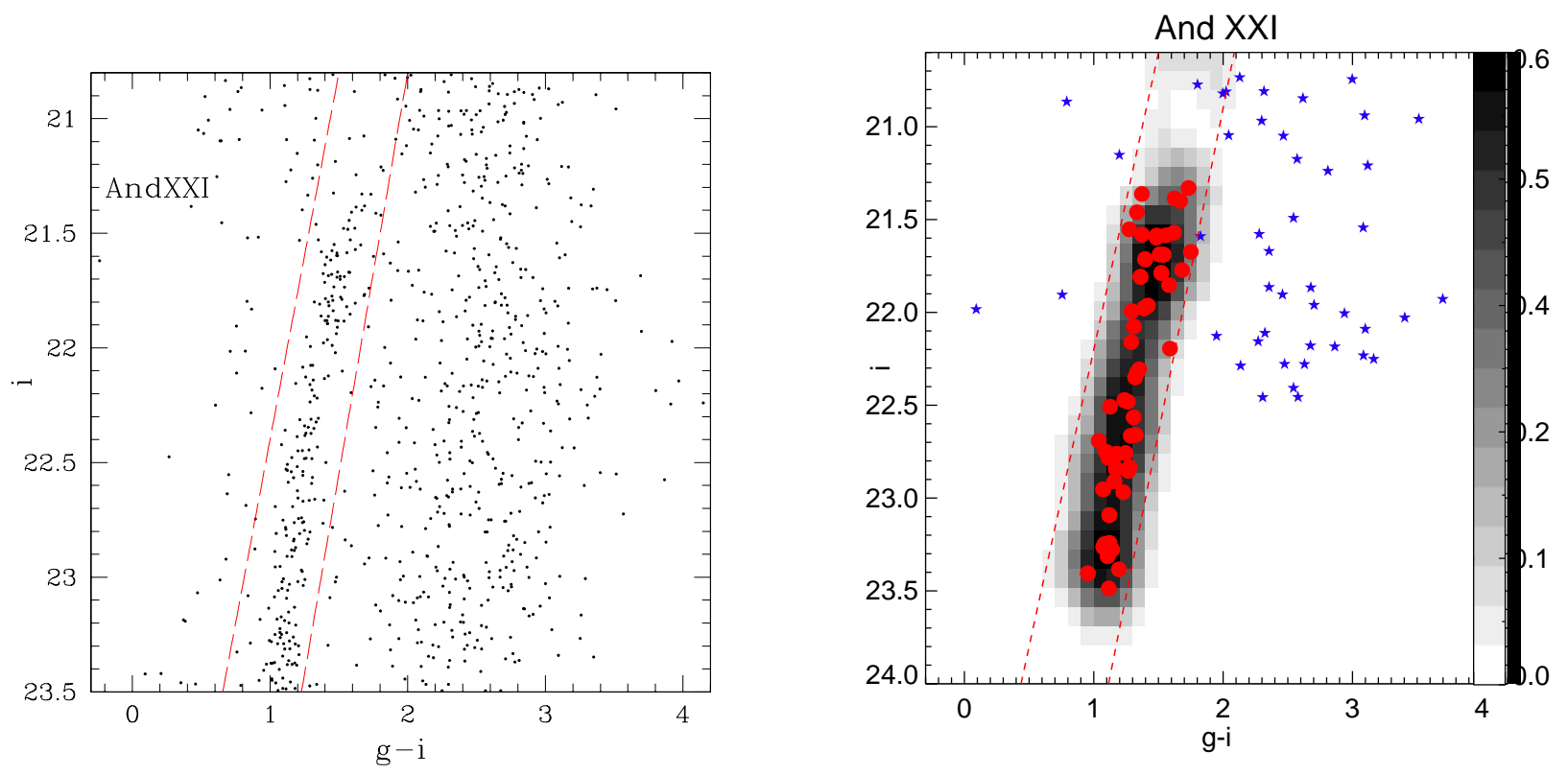

Fig. 2.- Left: The PAndAS CMD for And XXI, showing all stars within $2 \times r_{\text {half }}$ of the dwarf centre. The red dashed lines show the bounding box we use to construct our probability map for the RGB of the dSph. Right: A background normalized Hess diagram for the RGB region of And XXI. The color represents the probability, $P_{C M D}$ in each cell for a star in that position belonging to And XXI. This diagram is used as a probability map for all our spectroscopically observed stars. Red points represent stars DEIMOS stars for which $P_{C M D}>10^{-6}$, while the blue stars represent objects for which $P_{C M D} \leq 10^{-6}$.

$$
\begin{gathered}
P_{\text {halo }}=\frac{1}{\sqrt{2 \pi\left(\sigma_{v, \text { halo }}^{2}+v_{\text {err }, i}^{2}\right)}} \times \\
\exp \left[-\frac{1}{2}\left(\frac{v_{r, \text { halo }}-v_{r, i}}{\sqrt{\sigma_{v, \text { halo }}^{2}+v_{\text {err }, i}^{2}}}\right)^{2}\right]
\end{gathered}
$$

The MW halo population is well approximated by 2 Gaussians with $v_{r, M W 1}=-81.2 \mathrm{~km} \mathrm{~s}^{-1}, \sigma_{v, M W 1}=$ $36.5 \mathrm{~km} \mathrm{~s}^{-1}$ and $v_{r, M W 2}=-40.2 \mathrm{~km} \mathrm{~s}^{-1}$ and $\sigma_{v, M W 2}=$ $48.5 \mathrm{~km} \mathrm{~s}^{-1}$, resulting in a probability density function for a given star with a velocity $v_{i}$ and velocity uncertainty of $v_{e r r, i}$ of:

$$
\begin{array}{r}
P_{M W}=\frac{R}{\sqrt{2 \pi\left(\sigma_{v, M W 1}^{2}+v_{e r r, i}^{2}\right)}} \times \\
\exp \left[-\frac{1}{2}\left(\frac{v_{r, M W 1}-v_{r, i}}{\sqrt{\sigma_{v, M W 1}^{2}+v_{e r r, i}^{2}}}\right)^{2}\right] \\
+\frac{(1-R)}{\sqrt{2 \pi\left(\sigma_{v, M W 2}^{2}+v_{e r r, i}^{2}\right)}} \times \\
\exp \left[-\frac{1}{2}\left(\frac{v_{r, M W 2}-v_{r, i}}{\sqrt{\sigma_{v, M W 2}^{2}+v_{e r r, i}^{2}}}\right)^{2}\right]
\end{array}
$$

where $R$ is the fraction of stars in the first MW peak, and $(1-R)$ is the fraction of stars in the second peak. The value of $R$ is determined empirically from our DEIMOS data set.

A strong kinematic peak outside of these two populations can then be searched for using a maximum likeli- hood technique, based on the approach of Martin et al. (2007). We search for the maximum in the likelihood function that incorporates the two contamination populations plus an additional Gaussian structure with systemic velocity $v_{r, s u b s t r}$ and a dispersion of $\sigma_{v, s u b s t r}$, defined as:

$$
\begin{gathered}
P_{\text {substr }}=\frac{1}{\sqrt{2 \pi\left(\left[\eta \sigma_{v, \text { substr }}\right]^{2}+v_{\text {err }, i}^{2}\right)}} \times \\
\exp \left[-\frac{1}{2}\left(\frac{v_{r, \text { substr }}-v_{r, i}}{\left.\left.\sqrt{\left[\eta \sigma_{v, \text { substr }}\right]^{2}+v_{\text {err }, i}^{2}}\right)\right]}\right)^{2}\right]
\end{gathered}
$$

Here, to ensure we haven't biased our $P_{\text {substr }}$ strongly against stars that lie within the wings of the Gaussian distribution of dwarf spheroidal velocities, we have included a multiplicative free parameter, $\eta$, to our derived value of $\sigma_{v}$. To determine the ideal value of $\eta$, we ran our algorithm over all our datasets, changing the value of $\eta$ from $0.5-10.5$ to see its effect on the final derived systemic velocities and velocity dispersion. We find that in all cases, the solutions converge at values of $\eta \sim 2-4$. For dSphs whose kinematics are well separated from contaminants, the derived kinematics can remain stable up to much larger values of $\eta$, however for those with systemic velocities within the velocity regime of the Milky Way, the solution quickly destabilizes as more contaminants are included as probable members. We show this implicitly in Fig. 4, where we present the effect of modifying $\eta$ on 6 dSphs, And XII, XIX, XXI, XXII, XXIII and XXV. These objects were selected as they nicely probe our datasets with low numbers of probable member stars $(\sim 8)$, to those where we have 10s of probable members, as well as sampling dSphs from highly contaminated to well isolated kinematic regimes. The value of 


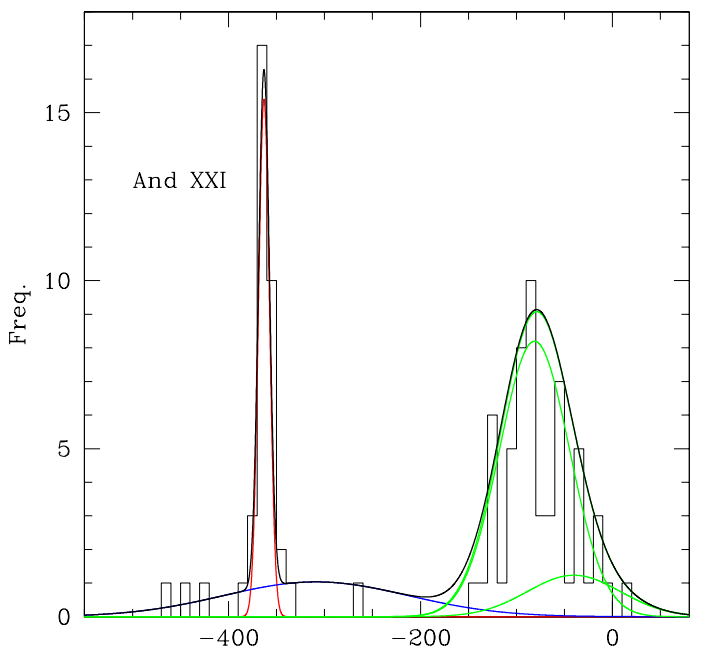

FIG. 3. - A velocity histogram for all observed stars in the field of And XXI. Our empirical Gaussian fits to the full Keck II data set for the M31 and MW halos are overlaid in blue and green respectively. A cold, kinematic peak at $-400 \mathrm{~km} \mathrm{~s}^{-1}$ is also seen, and this is the likely signature of the dSph. Our coarse, initial ML procedure identifies this peak, and the values of $v_{s y s}$ and $\sigma_{v}$ it measures are used to derive our kinematic probability, $P_{v e l}$.

$\eta$ is therefore independently determined for each dataset separately, and we report its final value in Table 4.

$$
\begin{gathered}
P_{\text {substr }}=\frac{1}{\sqrt{2 \pi\left(\sigma_{v, \text { substr }}^{2}+v_{\text {er }, i}^{2}\right)}} \times \\
\exp \left[-\frac{1}{2}\left(\frac{v_{r, \text { substr }}-v_{r, i}}{\left.\left.\sqrt{\sigma_{v, \text { substr }}^{2}+v_{\text {err }, i}^{2}}\right)\right]}\right)^{2}\right]
\end{gathered}
$$

The likelihood function can then be simply written as:

$\log \left[\mathcal{L}\left(\mathrm{v}_{\mathrm{r}}, \sigma_{\mathrm{v}}\right)\right]=\sum_{\mathrm{i}=1}^{\mathrm{N}} \log \left(\alpha \mathrm{P}_{\mathrm{i}, \text { halo }}+\beta \mathrm{P}_{\mathrm{i}, \mathrm{MW}}+\gamma \mathrm{P}_{\mathrm{i}, \text { substr }}\right)$

where $\alpha, \beta$ and $\gamma$ represent the Bayesian priors, i.e., the expected fraction of stars to reside in each population. These are determined by starting with arbitrary fractions (for example, $0.2,0.5$ and 0.3 respectively) and are then adjusted to the posterior distribution until priors and posteriors match. This technique will therefore identify an additional kinematic peak, independent of the MW and M31 halo populations, if it exists. We stress that these are not the final systemic velocity and dispersion of the dSph, but merely indicate a region in velocity space in which an excess of stars above the two contaminant populations is seen. In Fig. 3, we show the result of this process for the And XXI dSph. Here, the substructure is clearly visible as a cold spike at $\sim-400 \mathrm{~km} \mathrm{~s}^{-1}$.

Now that we have a velocity profile for our three components (MW, M31 halo and the dSph), we can assign probabilities for each star within our sample belonging to each population using simple Bayesian techniques, i.e., the probability that a given star belongs to the substructure, $P_{v e l}$, is:

$$
P_{\text {vel }}=\frac{\gamma P_{\text {substr }}}{\alpha P_{\text {halo }}+\beta P_{M W}+\gamma P_{\text {substr }}}
$$

and the probability of being a contaminant is:

$$
P_{\text {nvel }}=\frac{\alpha P_{\text {halo }}+\beta P_{M W}}{\alpha P_{\text {halo }}+\beta P_{M W}+\gamma P_{\text {substr }}}
$$

\subsection{Measuring $v_{r}$ and $\sigma_{v}$}

Upon applying this to our data, we can identify the most probable members of each $\mathrm{dSph}$, without having to apply any additional constraints or cuts.

Having established the membership probability for each observed star (as detailed above) we now calculate the kinematic properties of each dSph; namely their systemic velocities $\left(v_{r}\right)$ and velocity dispersions $\left(\sigma_{v}\right)$. We use the maximum likelihood technique of Martin et al. (2007), modified to include our probability weights for each star. We sample a coarse grid in $\left(v_{r}, \sigma_{v}\right)$ space and determining the parameter values that maximise the likelihood function (ML), defined as:

$\log \left[\mathcal{L}\left(v_{r}, \sigma_{v}\right)\right]=-\frac{1}{2} \sum_{i=1}^{N}\left[P_{i} \log \left(\sigma_{\text {tot }}^{2}\right)+P_{i}{\frac{v_{r}-v_{r, i}}{\sigma_{\text {tot }}}}^{2}+P_{i} \log (2 \pi)\right]$

where $N$ is the number of stars in the sample, $v_{r, i}$ is the radial velocity measured for the $i^{\text {th }}$ star, $v_{\text {err }, i}$ is the corresponding uncertainty and $\sigma_{\text {tot }}=\sqrt{\sigma_{v}^{2}+v_{e r r, i}^{2}}$. In this way, we aim to separate the intrinsic dispersion of a system from the dispersion introduced by the measurement uncertainties.

\subsection{Testing our probabilistic determination of membership and calculations of kinematic properties}

Having developed the above technique, it is important for us to rigorously test that it is robust enough to accurately determine the global kinematic properties for each of our datasets. In Appendix A, we examine in detail a number of potential issues that could cause our algorithm to return biased or incorrect results. These are the inclusion of a velocity dependent term in our probability calculation, the effect of including low S:N data $(\mathrm{S}: \mathrm{N}<5 \stackrel{\circ}{\mathrm{A}})$ in our analysis and the effect of small sample sizes $\left(N_{*}<8\right)$ on our measurements of kinematic properties. We briefly summarize our findings here, and refer the reader to Appendix A for a more detailed description.

This work has introduced the concept of assigning a probability of membership for a given star to a dSph based on the prior knowledge of the velocity profiles of our expected contaminant populations, $P_{v e l}$, a technique that has not previously been used in the study of M31 dSphs. To test that this is not biasing our results, we can simply remove this term from Eqn. 11 and follow the technique of T12, where they use only $P_{C M D}$ and $P_{d i s t}$ terms and then cut all stars with $P_{i}<0.1$ and velocities that lie greater than $3 \sigma$ from the mean of this sample from their final analysis. We find that both techniques produce very similar results, however our algorithm is 

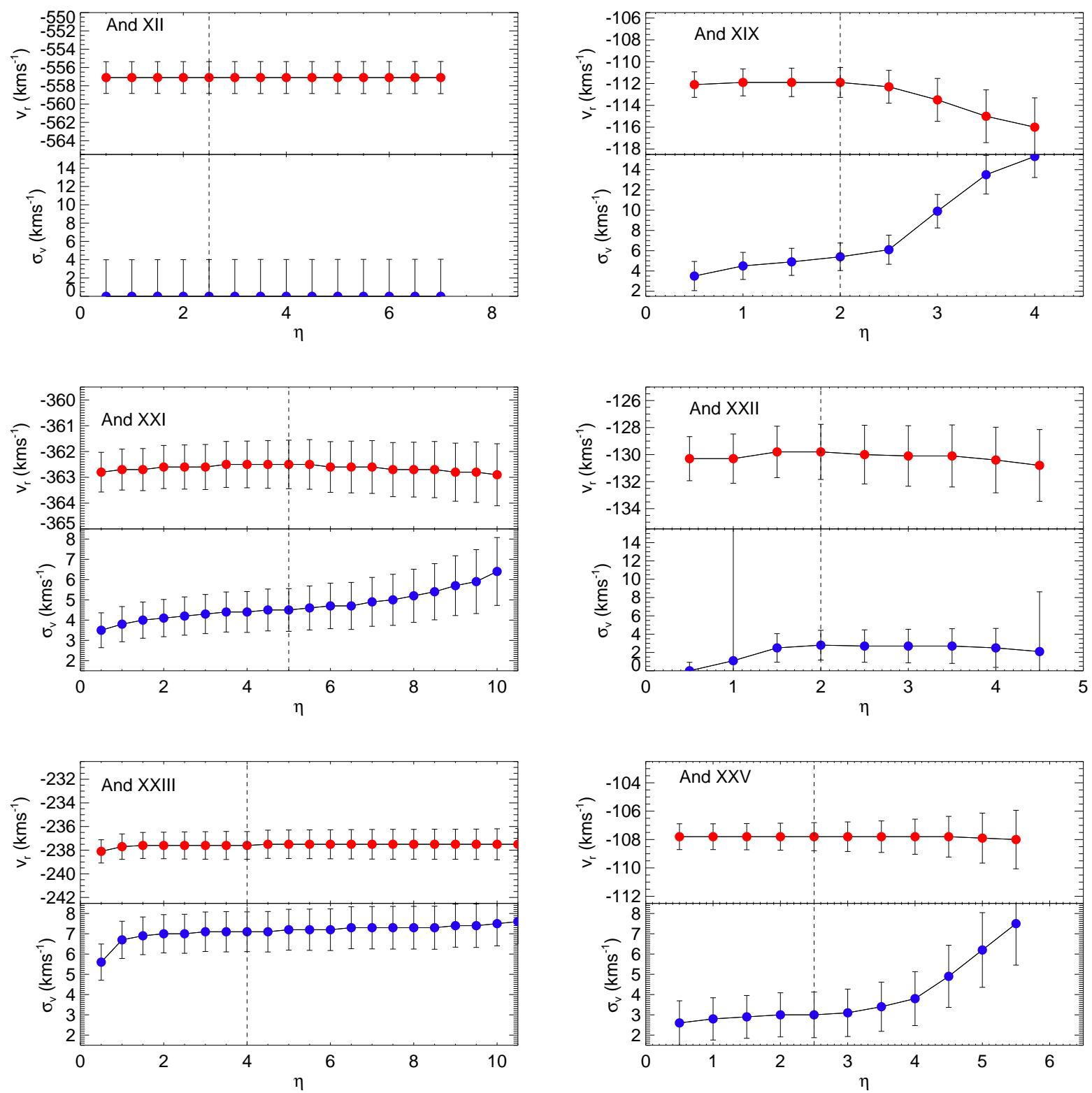

FIG. 4. - The effect of modifying $\eta$ - a multiplicative weight applied to the dispersion of the identified substructure in our $P_{v e l}$ term - on the final derived systemic velocities $\left(v_{r}\right)$ and dispersions $\left(\sigma_{v}\right)$ for six of our dSphs. The effect of increasing $\eta$ is typically more pronounced in objects with systemic velocities close to that of Milky Way contamination (such as And XIX and XXV) than for other objects. Dashed lines represent the optimal value of $\eta$ for each system.

more robust in regimes where the systemic velocity of the dSph is close to that of the MW, and in dSphs where our number of probable member stars is low $\left(N_{*}<10\right)$.

To test the effect of low S:N data on our calculations of $v_{r}$ and $\sigma_{v}$, we use our datasets for And XXI, XXIII and XXV, all of which have $\geq 25$ associated members. For each dataset, we apply a series of cuts to the sample based on S:N (at levels of $\mathrm{S}: \mathrm{N}>2,3,4$ and $5 \stackrel{\circ}{\mathrm{A}}$ ) and rerun our algorithm. In all cases we find that the derived probabilities do not significantly differ when the low S:N data are included, justifying our inclusion of all stars for which velocities are calculated by our pipeline.

We also test the ability of our algorithm to measure $v_{r}$ and $\sigma_{v}$ in the small $N_{*}$ regime. For some of our datasets, we are only able to identify a handful of stars as probable members. In theory, one can calculate velocity dispersions accurately from only 3 stars if one is confident of ones measurement uncertainties, as is demonstrated by Aaronson, (1983) measurement of the velocity dispersion for Draco from only 3 stars, which remains consistent with modern day measurements from significantly larger datasets (Walker et al. 2009b). We can test if our results are similarly robust using our larger datasets (such as And XXI, XXIII and XXV) by randomly selecting $4,6,8,10,15,20$ and 25 stars from these datasets and rerunning our algorithm to determine $v_{r}$ and $\sigma_{v}$ from these subsets. We repeat this exercise 1000 times for each sample size, and examine the mean and standard deviations for the computed quantities. We find that on aver- 
age, for all sample sizes, our routine measures systemic velocities and velocity dispersions that are entirely consistent with those measured from the full sample, with a spread that is very comparable to typical errors produced by our ML routine in these low $N_{*}$ regimes. As such, we conclude that our technique is able to place sensible limits on these values, even when dealing with as few as four member stars.

Finally, as the individual positions, velocities and velocity uncertainties for all the stars analyzed in T12 are publicly available (with the data from the non-member stars having been kindly passed on to us by the SPLASH team), we can check that our algorithm is able to reproduce the values they measure for their M13 dSph sample. We find that, in all cases, we calculate systemic velocities and velocity dispersions that agree with their measured values to well within their $1 \sigma$ uncertainties.

These tests demonstrate that our method is robust enough to accurately determine the global kinematic properties of M31 dSphs across a wide range of sample sizes and data quality. We therefore proceed to apply it to the datasets of all of the dSphs for which our group has acquired Keck II DEIMOS observations to date.

\section{THE KINEMATICS OF M31 DSPHS}

With this vast dataset of dSph kinematics now in hand, we can begin to statistically probe their structures more fully. In Fig. 5] we display a summary of the velocities and positions of 26 of the $28 \mathrm{dSphs}$ for which kinematic data are available, where the values are taken from this work, T12 and Kalirai et al. (2010). In the following sections, we will discuss the individual stellar kinematics, masses and chemistries of the dSphs analyzed within this work.

\subsection{Andromeda XVII}

And XVII was discovered by Irwin et al. (2008), and it is located at a projected distance of $\sim 40 \mathrm{kpc}$ to the North West of Andromeda. A detailed study of deep imaging obtained with the Large Binocular Camera on the LBT was also performed by Brasseur et al. (2011b), and throughout, we use the structural properties as determined from this work. It is a faint, compact galaxy $\left(M_{V}=-8.61, r_{\text {half }}=1.24^{\prime}\right.$ or $\left.262_{-46}^{+53} \mathrm{pc}\right)$. In the left panel of Fig. 6] we display the PAndAS color magnitude diagram for And XVII. Over-plotted we show the observed DEIMOS stars, color-coded by their probabilities of membership. The open symbols represent stars for which $P_{i}<10^{-6}$. We employ this cut solely to make clarify which stars have the highest probability of belonging to And XVII. In the right hand panel, we display the basic kinematic information for And XVII. In the top panel of this subplot, we show a velocity histogram for all stars observed within the LRIS mask, and stars with $P_{i}>10^{-6}$ are highlighted with a filled red histogram. The centre panel shows the velocities as a function of distance from the centre of And XVII (and the red dashed lines indicate $1,2,3$ and $4 \times r_{\text {half }}$ ), and the lower panel shows the photometric metallicities for all stars, as determined using Dotter et al. (2008) CFHT isochrones. Again, all points are color-coded by their probability of membership. Finally, the two lower panels show the resulting, one dimensional, probability weighted, marginalized maximum likelihood distributions for $v_{r}$ and $\sigma_{v}$ for this data set. From the kinematics presented in Fig. 6. which represent the first spectroscopic observations of this object, we see the signature of the dwarf galaxy as a cold spike at $v_{r} \sim-250 \mathrm{~km} \mathrm{~s}^{-1}$. From the lower panels of this figure and the accompanying CMD we see that there is a cluster of 7 stars sitting within this spike that are centrally concentrated and are consistent with the RGB of the dwarf itself, leading us to believe that our algorithm has cleanly detected the signature of the galaxy. Interestingly, we also see 3 stars that, kinematically, are indistinguishable from the stars that have been dubbed as probable members in our analysis. However, they all sit at large distances from the centre of And XVII, equivalent to greater than 6 times the half-light radius of the dwarf, and hence the routine has classified them as likely members of the M31 halo rather than And XVII members. But, given their tight correlation in velocity to the systemic velocity of And XVII the possibility exists that these are extra-tidal stars of And XVII. No sign of extratidal features were cited in either the discovery paper of And XVII or the LBT followup, but given its position in the north M31 halo where contamination from the MW becomes increasingly problematic, and its relatively low luminosity $\left(M_{V}=-8.5\right)$, such features may be difficult to see within the imaging. However, at present they are considered unlikely members by our routine, and do not factor into our calculation of global kinematic properties for this object. We find $v_{r}=-251.6_{-2.0}^{+1.8} \mathrm{~km} \mathrm{~s}^{-1}$ and $\sigma_{v}=2.9_{-1.9}^{+2.2} \mathrm{~km} \mathrm{~s}^{-1}$.

\subsection{Andromeda XVIII}

Andromeda XVIII (And XVIII) was detected by McConnachie et al. (2008) in the PAndAS CFHT maps. Located at a projected distance of $\sim 110 \mathrm{kpc}$ to the North-West of M31, it is one of the most distant of its satellites, sitting $\sim 600 \mathrm{kpc}$ behind the galaxy, making spectroscopic observations of its individual RGB stars taxing, as they are all relatively faint $(i \gtrsim 22.2)$. Thus, from our 1 hour DEIMOS observation, we were only able to confirm 4 stars as probable members (see Fig. 7). We determine the global systemic velocity to be $v_{r}=$ $-346.8 \pm 2.0 \mathrm{~km} \mathrm{~s}^{-1}$, and we are unable to resolve a velocity dispersion, finding $\sigma_{v}=0.0^{+2.7} \mathrm{~km} \mathrm{~s}^{-1}$ where the upper bound is determined from the formal $1 \sigma$ confidence interval produced by our maximum likelihood analysis. This suggests that the 4 stars we are able to confirm as members do not adequately sample the underlying velocity profile. The systemic velocity we measure is different to that presented in T12 of $v_{r}=-332.1 \pm 2.7$ at a level of $3.4 \sigma$. Our $1 \sigma$ limit of $2.7 \mathrm{kms}^{-1}$ is also at odds with the dispersion determined by T12 $\left(\sigma_{v}=9.7 \pm 2.3\right)$. They were able to measure velocities for significantly more probable member stars (22 vs. 4) owing to their longer integration of 3 hours. The faintness of our targets and shorter exposure time could mean that strong sky absorption lines have systematically skewed our velocity measurements for these stars, and this could explain the discrepancy of our measurements with respect to those of T12. To check against this, we again perform weighted cross-correlations to each of the $3 \mathrm{Ca}$ II lines individually. We find that the values we obtain, and their average, are fully consistent with that derived from the technique described in $\S 2.2$, differing by less than $3 \mathrm{kms}^{-1}$ from those values. The true systemic velocity 


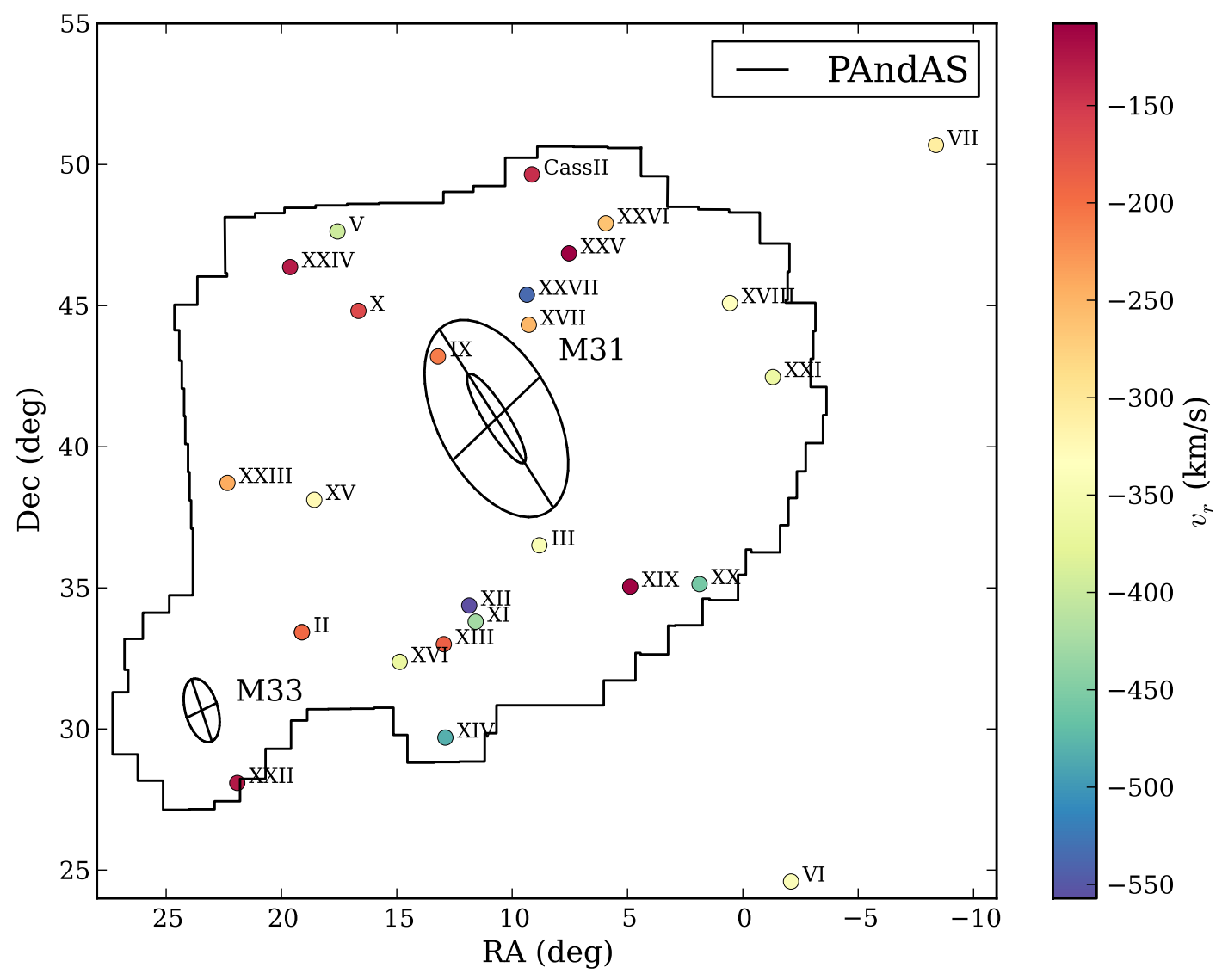

FIG. 5.- Positions of 26 of the 28 Andromeda dSphs, color coded by their heliocentric velocity, $v_{r}$ (taken from this work, Kalirai et al. 2010 and T12). The solid line represents the PAndAS survey foot print. M31 is shown by the double ellipse, where the outer ellipse shows a segment of a $55 \mathrm{kpc}$ radius ellipse flattened to $\mathrm{c} / \mathrm{a}=0.6$, and the major-axis and minor-axis are indicated with straight lines out to this ellipse. The inner ellipse corresponds to a disc of radius $2^{\circ}(27 \mathrm{kpc})$, with the same inclination as the main M31 disc. M33 is shown as a singular ellipse.

of And XVIII therefore remains unclear. However, given their larger sample size, the T12 systemic properties are more statistically robust than those we present here.

\subsection{Andromeda XIX}

Andromeda XIX (And XIX) was first reported in McConnachie et al. (2008), and is a relatively bright, very extended $\left(M_{V}=-9.3, r_{\text {half }}=1.5 \mathrm{kpc}\right) \mathrm{dSph}$, located at a projected distance of $\sim 180 \mathrm{kpc}$ to the south west of M31. Its unusual morphology, very low surface brightness $\Sigma_{v}=30.2 \mathrm{mag} / \operatorname{arcsec}^{2}$, and evidence in the photometry for a possible link to the major axis substructure reported in Ibata et al. (2007) caused McConnachie et al. (2008) to question whether And XIX was truly a dynamically relaxed system, or whether it had experienced a significant tidal interaction. Here, we present the first spectroscopic observations of the And XIX satellite in Fig. 8 from two DEIMOS masks placed at different position angles. These data allow us to comment on its dark matter content, and on the likelihood of a tidal origin for its unique structure. We identify 27 stars where $P_{i}>10^{-6}$ within the system. These measurements were made increasingly challenging as the systemic velocity we measure is $v_{r}=-111.6_{-1.4}^{+1.6} \mathrm{~km} \mathrm{~s}^{-1}$, placing it within the regime of Galactic contamination. However, we are confident that our algorithm is robust to this unfortunate location of And XIX in velocity space (see discussion in $\S 3.5$ and Appendix A). As a further check that none of the stars we define as probable members are actually foreground contaminants, we measure the equivalent widths of the $\mathrm{Na}$ I doublet lines (located at $\sim 8100 \AA$ ). These gravity-sensitive absorption lines are typically significantly stronger in foreground dwarf stars than M31 RGB stars, although there is some overlap between the two populations. For the stars tagged as probable members by our algorithm, we find no evidence of strong absorption in the region of the $\mathrm{Na}$ I doublet, indicating that we are not selecting foreground stars as members. We measure a relatively cold velocity dispersion for this object of $\sigma_{v}=4.7_{-1.4}^{+1.6} \mathrm{~km} \mathrm{~s}^{-1}$, which is surprising given the radial extent of this galaxy. This result will be discussed further in $\S 5.1$, and in a follow-up paper (Collins et al. in prep).

\subsection{Andromeda $X X$}



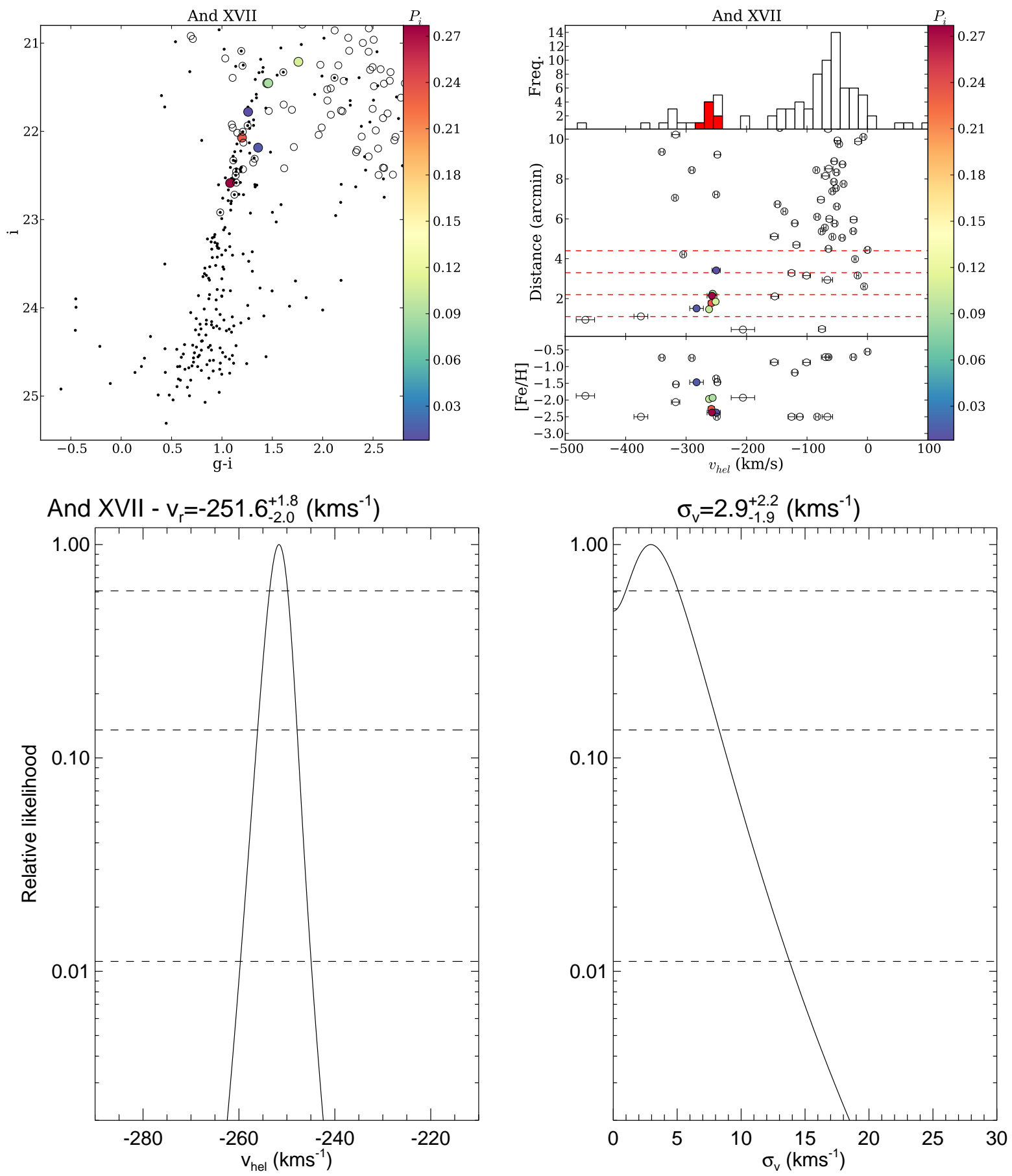

FIG. 6.- CMD of And XVII, from PAndAS photometry (not extinction corrected). All stars observed with DEIMOS are color coded by their probability of membership. Top right: Kinematic information for all stars observed with DEIMOS. The top subplot shows a velocity histogram for our sample, with all stars with $P_{i}>10^{-6}$ highlighted as a filled red histogram. The central subplot shows the distance of each star from the centre of And XVII as a function of velocity, and the lower subplot shows the photometric metallicity for each star, interpolated from Dotter et al. (2008) isochrones in the CFHT-MegaCam $g$ - and $i$-bands (after correcting the observed colors of the stars for extinction using the Schlegel et al. (1998) dust maps), as a function of velocity. Stars that lie far from the RGB of the dSph are not well matched by the isochrones, and as a result there is no estimate of their $[\mathrm{Fe} / \mathrm{H}]$. Lower panels: Resulting probability distributions from ML analysis of the kinematics of And XVII. The left hand panel shows the systemic velocity $\left(v_{r}\right)$ likelihood distribution and the right shows the intrinsic velocity dispersion $\left(\sigma_{v}\right)$ likelihood distribution. The dashed lines represent canonical 1,2 and $3 \sigma$ confidence intervals, derived assuming Gaussian uncertainties. 

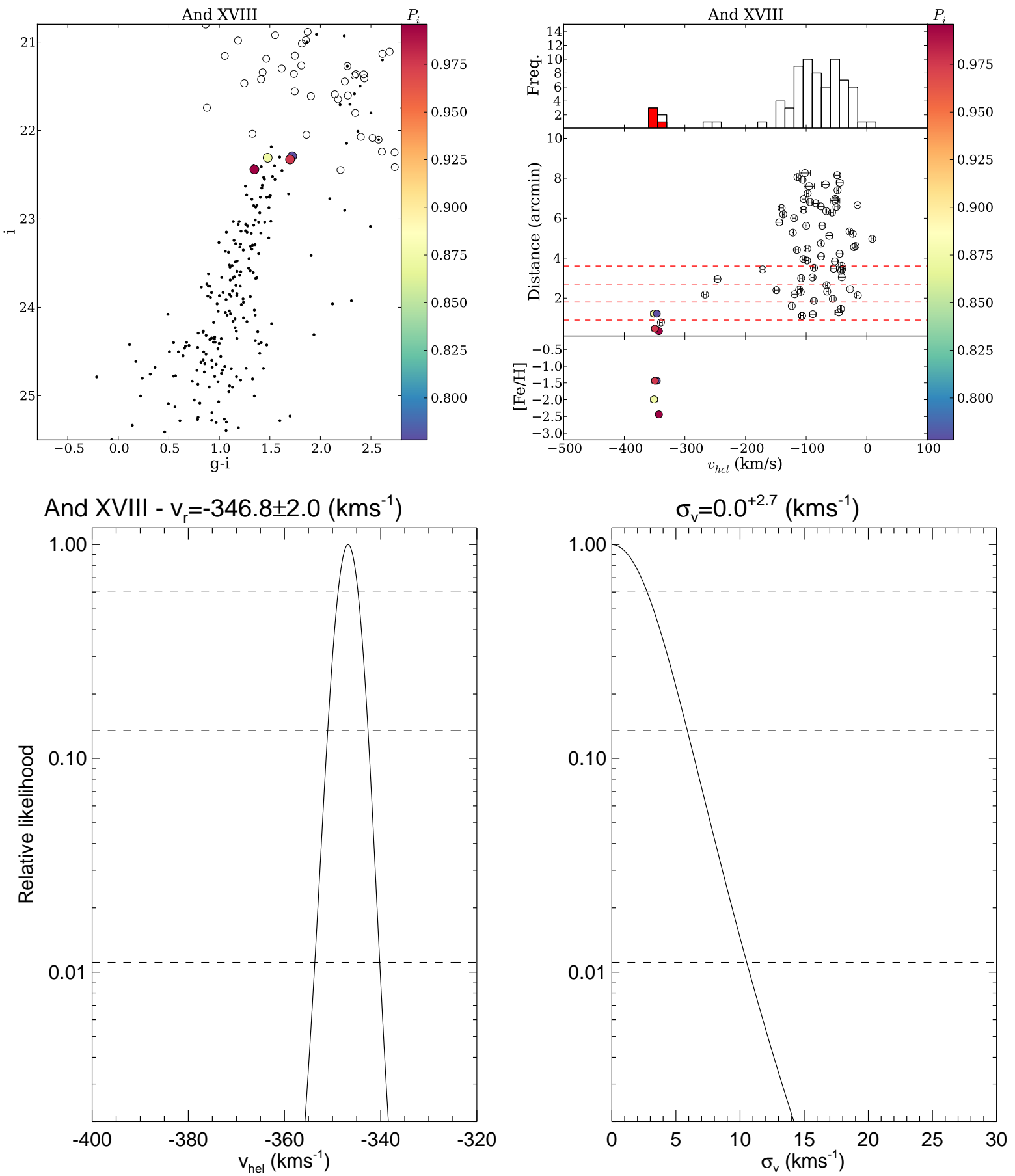

Fig. 7.- As Fig. 6 but for And XVIII. 

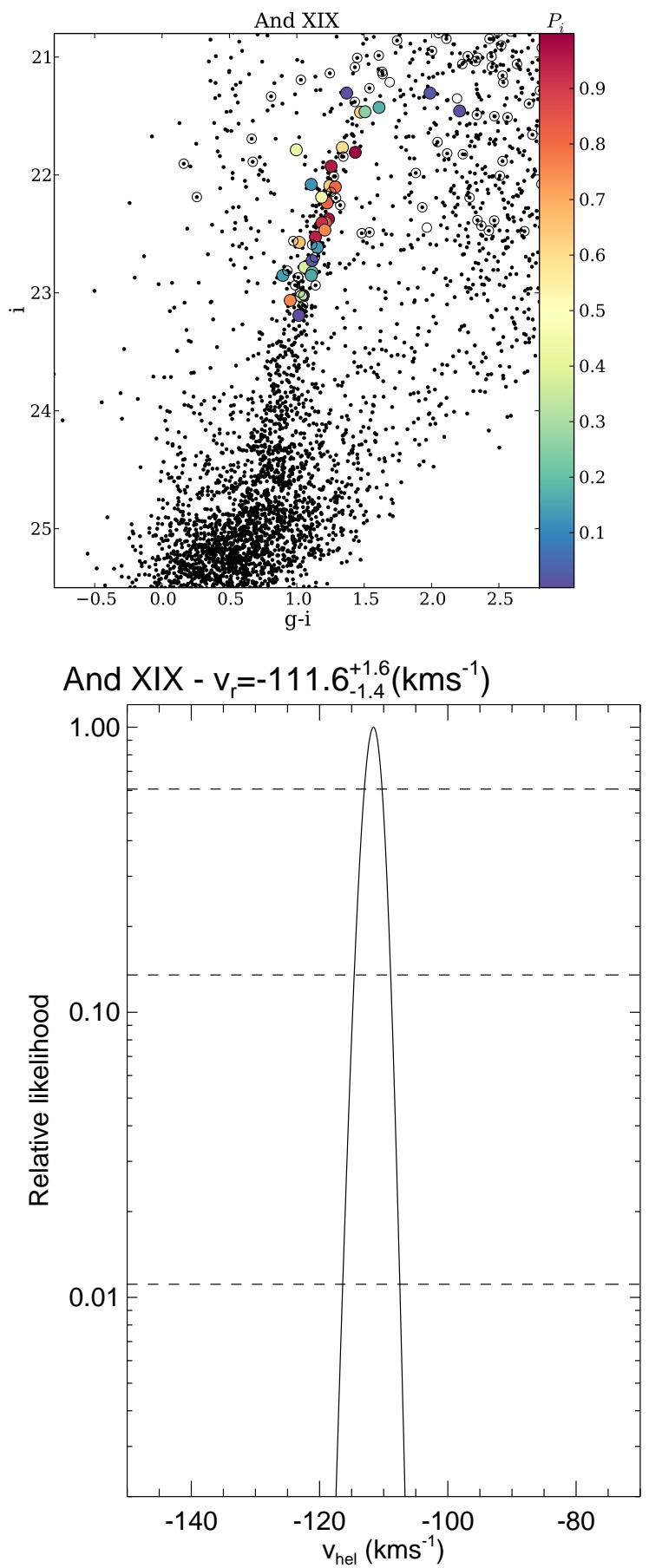

FIG. 8.- As Fig. 6 but for And XIX.

And XX was the third of three dSphs discovered by McConnachie et al. (2008), and is notable for being one of the faintest dSph companions detected surrounding Andromeda thus far. With $M_{V}=-6.3$ and $r_{\text {half }}=$ $114_{-12}^{+31} \mathrm{pc}$, it is a challenging object to study spectroscopically as there are very few stars available to target on its RGB, as shown in the top left subplot in Fig. 9 As a result, our algorithm is only able to find 4 stars for which $P_{i}>10^{-6}$. These are found to cluster around $v_{r}=-456.2_{-3.6}^{+3.1} \mathrm{~km} \mathrm{~s}^{-1}$, with a dispersion of
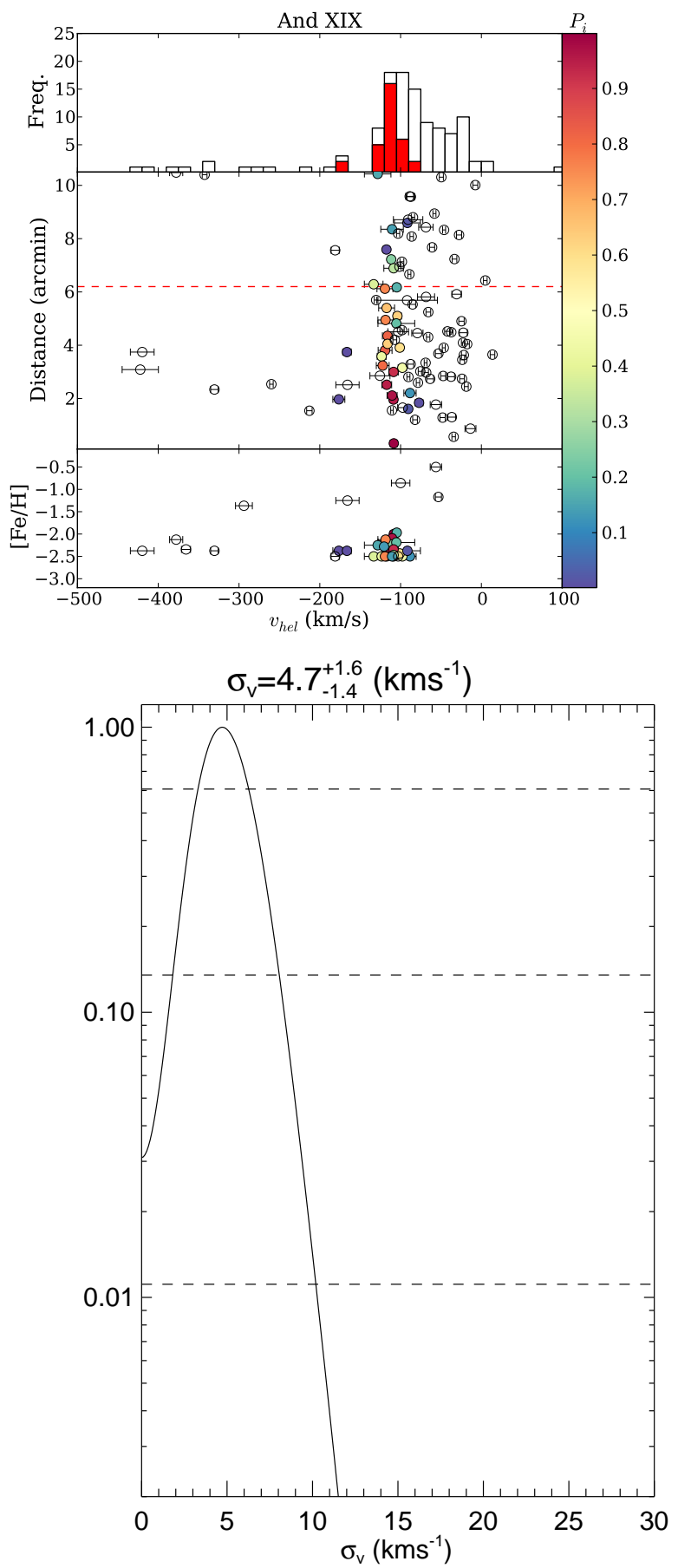

$\sigma_{v}=7.1_{-2.5}^{+3.9} \mathrm{~km} \mathrm{~s}^{-1}$. Despite the low number of stars, we are confident in this detection, as the systemic velocity places it in the outer wings of the velocity profile of the M31 halo. And XX is also located at a large projected distance from M31 of $\sim 130 \mathrm{kpc}$, where we expect the density of the M31 halo to be very low. As such, seeing 4 halo stars so tightly correlated in velocity in the wings of the halo velocity profile within such a small area of the sky (all stars are within 1 arcmin of the centre of And $\mathrm{XX}$ ) is highly unlikely. We caution the reader that, while 
we are confident that our algorithm is able to measure velocity dispersions for sample sizes as small as 4 stars, as we are not probing the full velocity profile of this object this measurement ideally needs to be confirmed with larger numbers of member stars.

\subsection{Andromeda XXI}

Andromeda XXI (And XXI) was identified within the PAndAS imaging maps by Martin et al. (2009). It is a relatively bright $\mathrm{dSph}\left(M_{V}=-9.9\right)$, located at a projected distance of $\sim 150 \mathrm{kpc}$ from M31, and it has a half-light radius of $r_{\text {half }}=842 \pm 77$ pc. We present our spectroscopic observations for this object in Fig. 10, and in the top right subplot, we can clearly see the signature of And XXI as a cold spike in velocity with 32 probable member stars, located at $v_{r}=$ $-362.5 \pm 0.9 \mathrm{kms}^{-1}$, with a curiously low velocity dispersion of only $\sigma_{v}=4.5_{-1.0}^{+1.2} \mathrm{~km} \mathrm{~s}^{-1}$. These results are completely consistent with those of T12, where they measured $v_{r}=-361.4 \pm 5.8 \mathrm{~km} \mathrm{~s}^{-1}$ and $\sigma_{v}=7.2 \pm 5.5 \mathrm{~km} \mathrm{~s}^{-1}$. As their sample contained only 6 likely members compared with the 29 we identify here, ours constitute a more statistically robust measurement of the global kinematics for this object than those presented in T12.

\subsection{Andromeda XXII}

Andromeda XXII (And XXII) was identified within the PAndAS imaging maps by Martin et al. (2009), and is a relatively faint $\mathrm{dSph}$, with $M_{V}=-6.5$. Its physical position in the halo, located at a distance of $224 \mathrm{kpc}$ in projection from M31, but only $42 \mathrm{kpc}$ in projection from M33, led the authors to postulate that it could be the first known dSph satellite of M33. Subsequent work analysing the kinematics of And XXII by T12 measured a systemic velocity for And XXII of $-126.8 \pm 3.1 \mathrm{~km} \mathrm{~s}^{-1}$ from 7 stars, more compatible with the systemic velocity of M33 (-178 $\mathrm{km} \mathrm{s}^{-1}$, Mateo 1998) than that of M31. Another study by Chapman et al. (2012) using the same data and the same method we present here concluded the same, measuring a systemic velocity for the satellite of $-129.8 \pm 2.0$ from 12 probable member stars, consistent with the T12 value. Chapman et al. (2012) also compare the position and kinematics of And XXII with a suite of $N$-body simulations of the M31-M33 system, concluding that And XXII was a probable M33 satellite.

In Fig. 11] we present the same data as analyzed by Chapman et al. (2012) for completeness. The velocity dispersion of And XXII is just resolved at $\sigma_{v}=$ $2.8_{-1.4}^{+1.9} \mathrm{~km} \mathrm{~s}^{-1}$, completely consistent with the value of $\sigma_{v}=3.5_{-2.5}^{+4.2} \mathrm{~km} \mathrm{~s}^{-1}$ from T12. As our values are calculated from a $50 \%$ greater sample size, we posit that they are the more statistically robust.

\subsection{Andromeda XXIII}

Andromeda XXIII (And XXIII) was the first of five M31 dSphs identified by Richardson et al. (2011). Located at a projected distance of $\sim 130 \mathrm{kpc}$ to the east of Andromeda, it is relatively bright, with $M_{V}=-10.2$, and extended, with $r_{\text {half }}=1001_{-52}^{+53}$ pc. Our routine clearly detects a strong cold kinematic peak for And XXIII located around $-230 \mathrm{~km} \mathrm{~s}^{-1}$ and calculates a systemic velocity of $v_{r}=-237.7 \pm 1.2 \mathrm{~km} \mathrm{~s}^{-1}$, and a velocity dispersion of $\sigma_{v}=7.1 \pm 1.0 \mathrm{~km} \mathrm{~s}^{-1}$ from 40 probable member stars, as show in Fig. 12. This small, positive velocity relative to $\mathrm{M} 31$, combined with its large projected distance from the host suggests that And XXIII is not far past the apocentre of its orbit, heading back towards M31.

\subsection{Andromeda XXIV}

And XXIV was also first reported in Richardson et al. (2011). Relatively faint and compact $\left(M_{V}=-7.6\right.$, $\left.r_{\text {half }}=548_{-37}^{+31} \mathrm{pc}\right)$, spatially it is located $\sim 200 \mathrm{kpc}$ from M31, along its northern major axis. And XXIV was observed on two separate occasions as detailed in Table 2 For the first mask, there was an error in target selection, and as a result, only one star that lay on the RGB of And XXIV was observed. The second mask was observed in May 2011, however owing to target visibility, only a short integration of 45 minutes was obtained, which resulted in higher velocity uncertainties than typically expected $\left(\sim 8 \mathrm{~km} \mathrm{~s}^{-1}\right.$ vs. $\left.\sim 5 \mathrm{~km} \mathrm{~s}^{-1}\right)$. For this reason, we have only included stars from this mask with $i<22.0$, as the spectra for fainter stars were too noisy to determine reliable velocities from. The systemic velocity of And XXIV also unsatisfactorily coincides with that of the MW halo contamination, as can be seen in Fig. 13. As for And XIX, we check the strength of the $\mathrm{Na} I$ doublet of all the stars classified as potential members for And XXIV, and find no significant absorption, making them unlikely foreground contaminants. But, given the lower quality of this dataset, this check is far from perfect, and it is possible that we have included contaminants from the MW within our sample. Owing to the larger velocity uncertainties of the And XXIV dataset, and the overlap of And XXIV with the MW, the determination of probability of membership for stars within this dataset is based largely on their position in the color magnitude diagram (e.g., location on the RGB) and their distance from the centre of And XXIV.

When we run our machinery over the data acquired from both masks, we identify only 3 probable members and determine a systemic velocity of $v_{r}=-128.2 \pm$ $5.2 \mathrm{~km} \mathrm{~s}^{-1}$ and we resolve a velocity dispersion of $\sigma_{v}=$ $0.0^{+7.3} \mathrm{~km} \mathrm{~s}^{-1}$. Given the lower quality of this dataset in comparison to the remainder of those we present in this work, and the overlap of And XXIV in velocity space with contamination from the MW, a robust kinematic detection and characterisation of this galaxy is made incredibly challenging. As such, we present these results as a tentative identification of And XXIV, and do not include its measured properties in the remainder of our analysis. Further kinematic follow up of And XXIV is required to understand this system. We present the velocities of all bright stars for which velocity measurements were possible in Table 3 so that they may be helpful for any future kinematic analysis of this system.

\subsection{Andromeda $X X V$}

And XXV was identified in Richardson et al. (2011) as a relatively bright $\left(M_{V}=-9.7\right)$, extended $\left(r_{\text {half }}=\right.$ $642_{-74}^{+47} \mathrm{pc}$ ) dwarf spheroidal, located at a projected distance of $\sim 90 \mathrm{kpc}$ to the north west of M31. As with And XXIII, we present here a kinematic analysis of And XXV. The results are displayed in Fig. 14. We see that the systemic velocity of And XXV $\left(v_{r}=-107.8 \pm 1.0 \mathrm{~km} \mathrm{~s}^{-1}\right)$, 

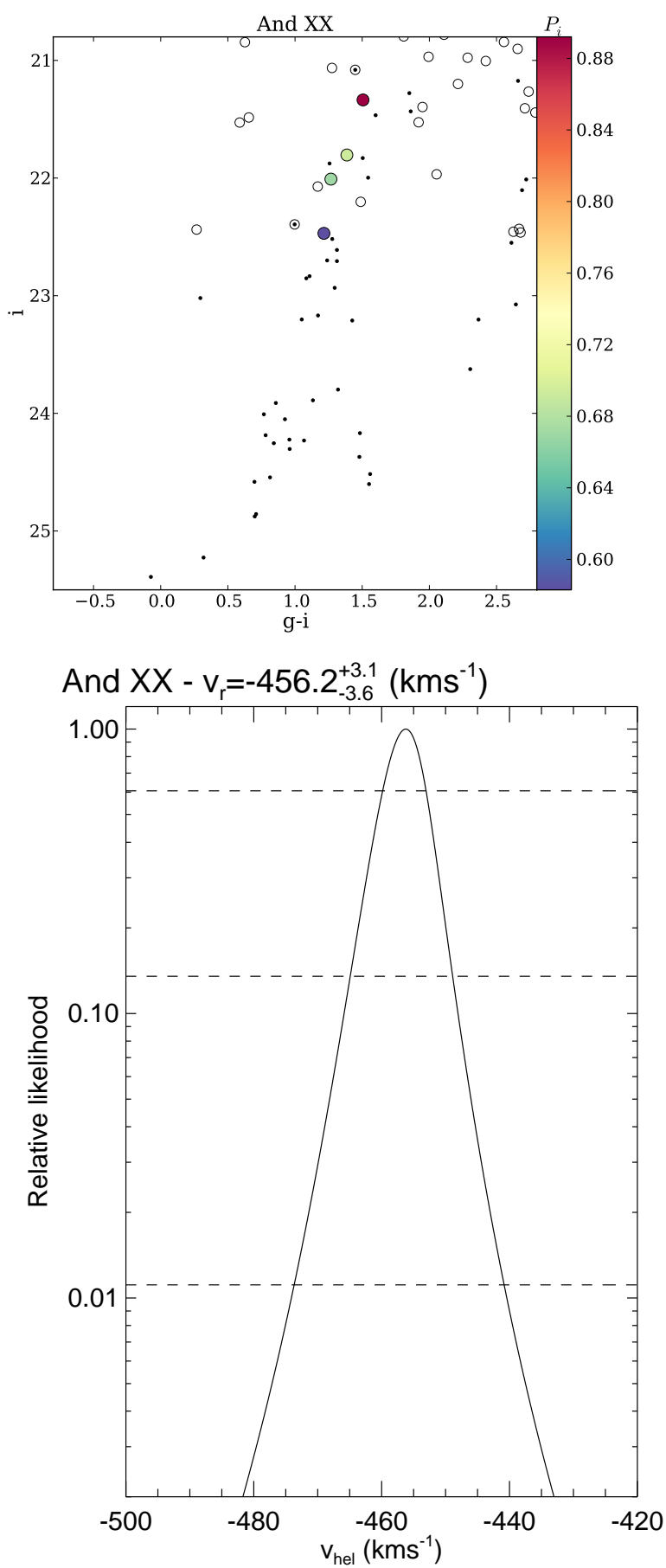

FIG. 9.- As Fig. 6 but for And XX.

places it in the regime of the Galactic foreground. However, given the strong over-density of stars with this velocity relative to the expected contribution of MW stars, we are confident that our routine has detected 25 likely members for this object. We check the strength of the Na I doublet in these likely members, and find no significant absorption, making them unlikely foreground contaminants. As for And XIX and XXI, we find that And XXV has a curiously low velocity dispersion for its size, with $\sigma_{v}=3.0_{-1.1}^{+1.2} \mathrm{~km} \mathrm{~s}^{-1}$. We discuss the significance of this
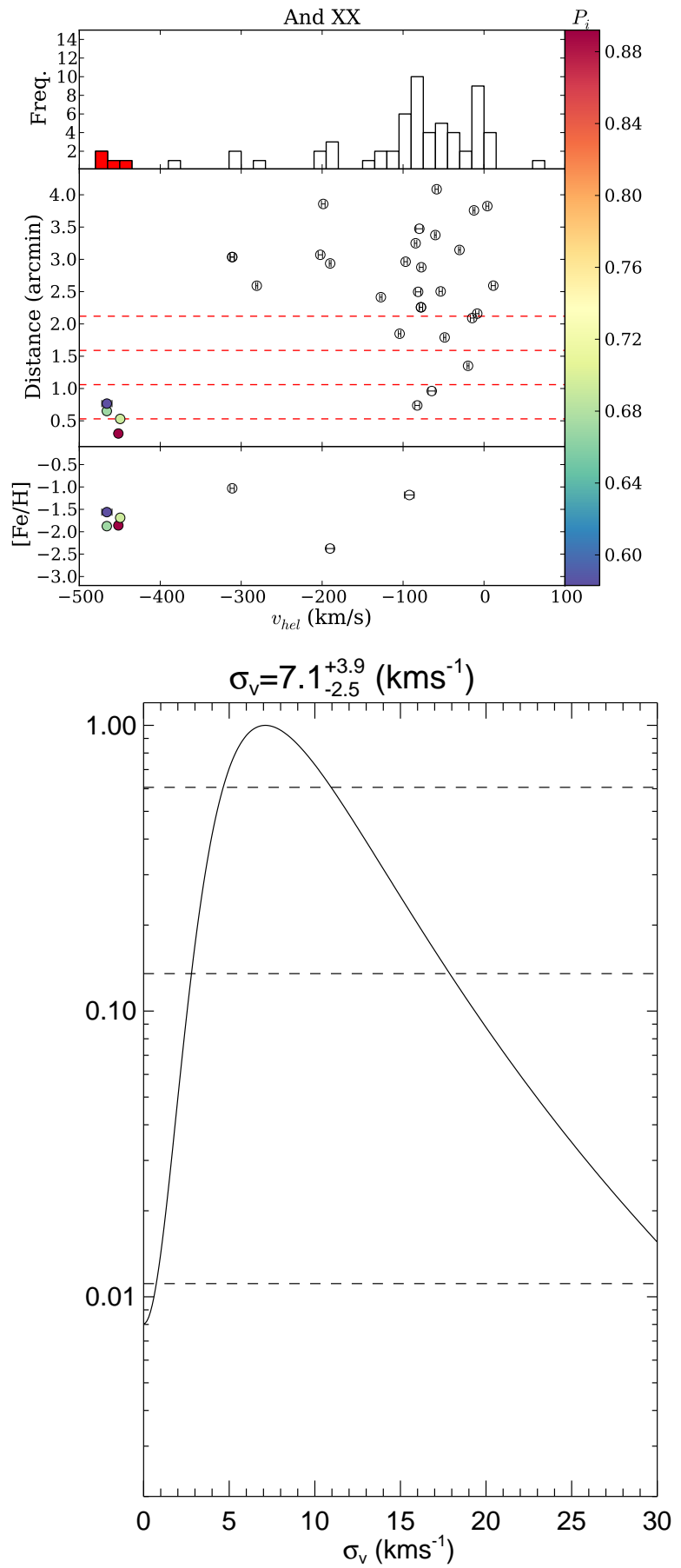

further in $\S 5.1$ and Collins et al (2013, in prep).

\subsection{Andromeda XXVI}

And XXVI is a relatively faint $\left(M_{V}=-7.1\right) \mathrm{dSph}$ with $r_{\text {half }}=219_{-52}^{+67} \mathrm{pc}$, also first reported in Richardson et al. (2011). Its low luminosity makes observing large numbers of member stars difficult, owing to the paucity of viable targets on the RGB that can be observed with DEIMOS. As a result, our routine has identified only 6 stars as potential members, highlighted in 

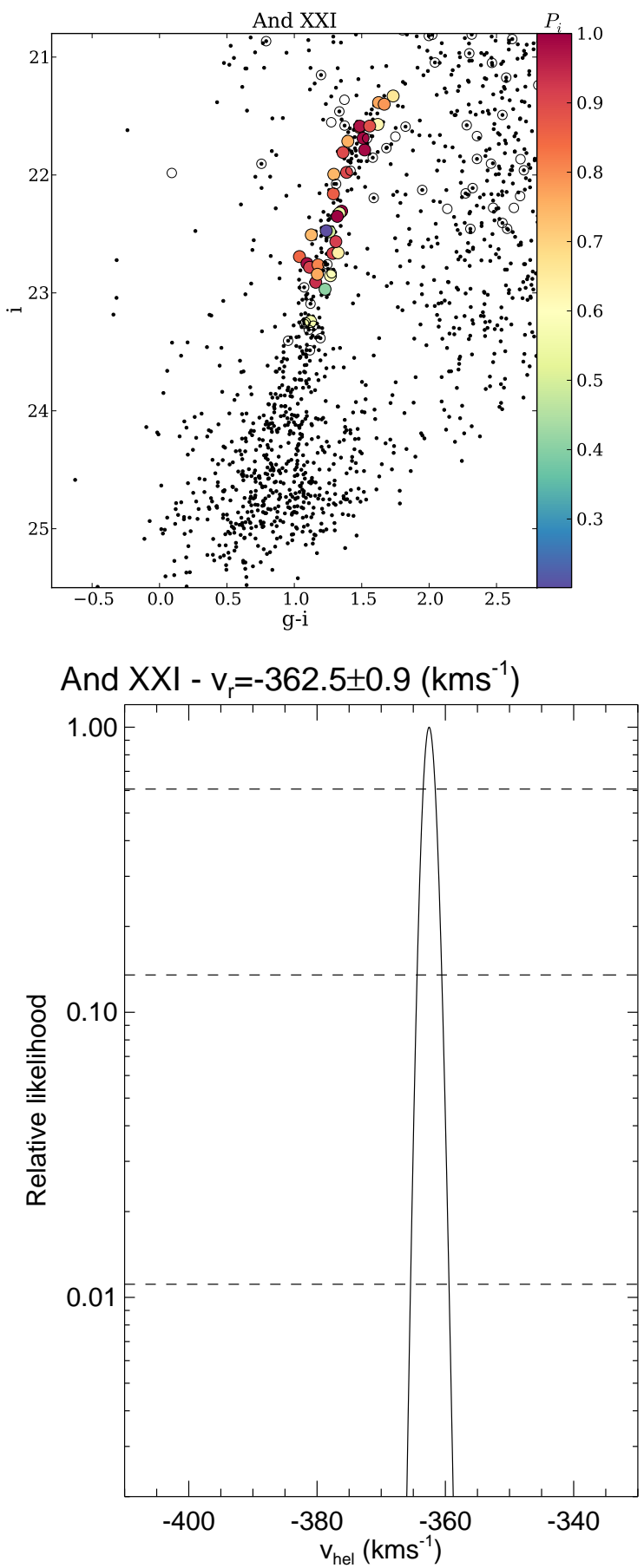

FIG. 10.- As Fig. 6 but for And XXI

Fig. 15. The dwarf has a systemic velocity of $v_{r}=$ $-261.6_{-2.8}^{+3.0} \mathrm{~km} \mathrm{~s}^{-1}$, and a fairly typical velocity dispersion of $\sigma_{v}=8.6_{-2.2}^{+2.8} \mathrm{~km} \mathrm{~s}^{-1}$. As with And XX, while we believe our routine can robustly measure the velocity dispersions of systems with only 6 confirmed members, to be truly confident of this value, follow up of And XXVI to increase the number of likely members is required.

In Conn et al. (2012), from an analysis of the photometry of And XXVI, they determined a distance modulus
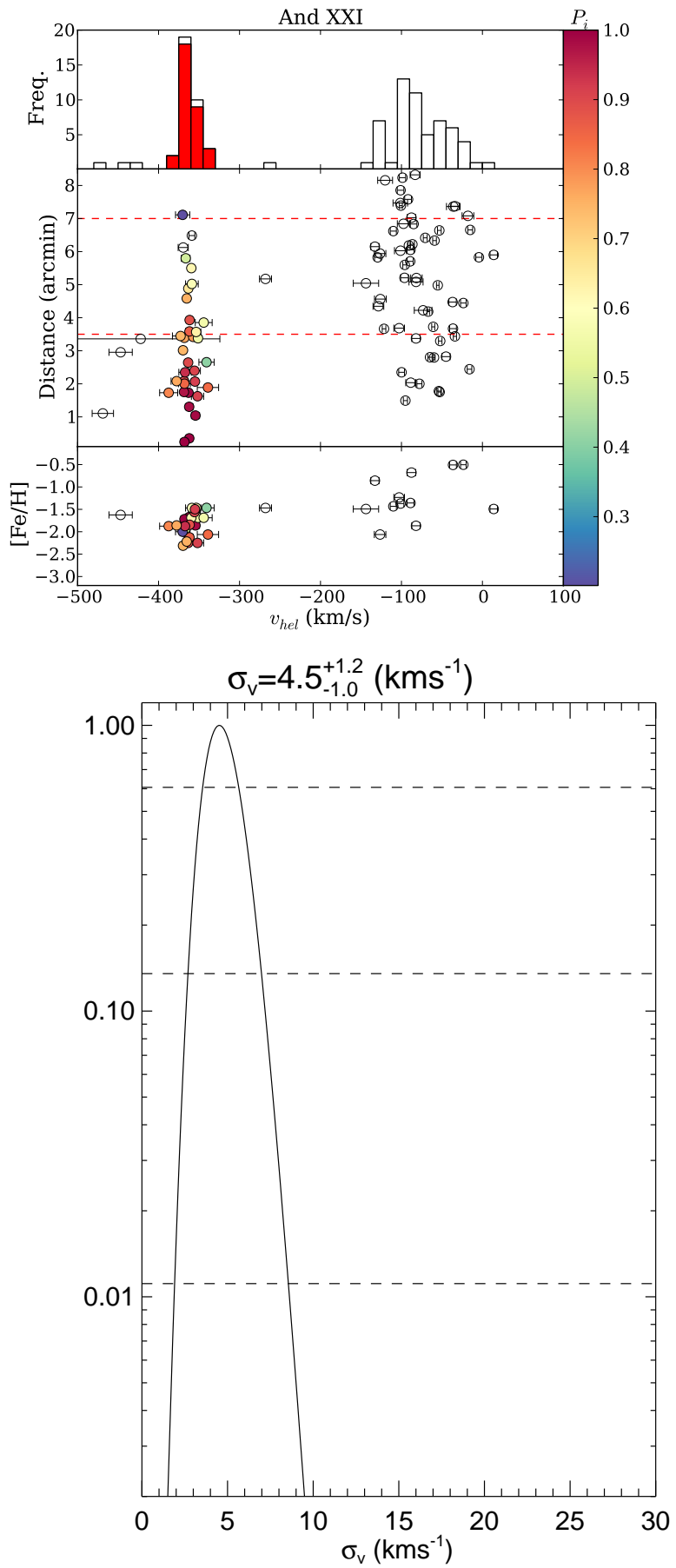

to the object of $(m-M)_{0}=24.39_{-0.53}^{+0.55}$ from a MarkovChain-Monte-Carlo analysis of the PAndAS photometry of And XXVI. This value corresponds to an $i$-band magnitude for the TRGB of And XXVI of $m_{i, 0}=21.1_{-0.53}^{+0.55}$. Our CMDs for the dwarfs are not extinction corrected, but using the extinction values from Richardson et al. (2011) of $E(B-V)=0.110$ (Schlegel et al. 1998), this would correspond to an $i$-band magnitude of $m_{i, T R G B}=$ $21.3_{-0.53}^{+0.55}$. Three targets were observed with magnitudes 

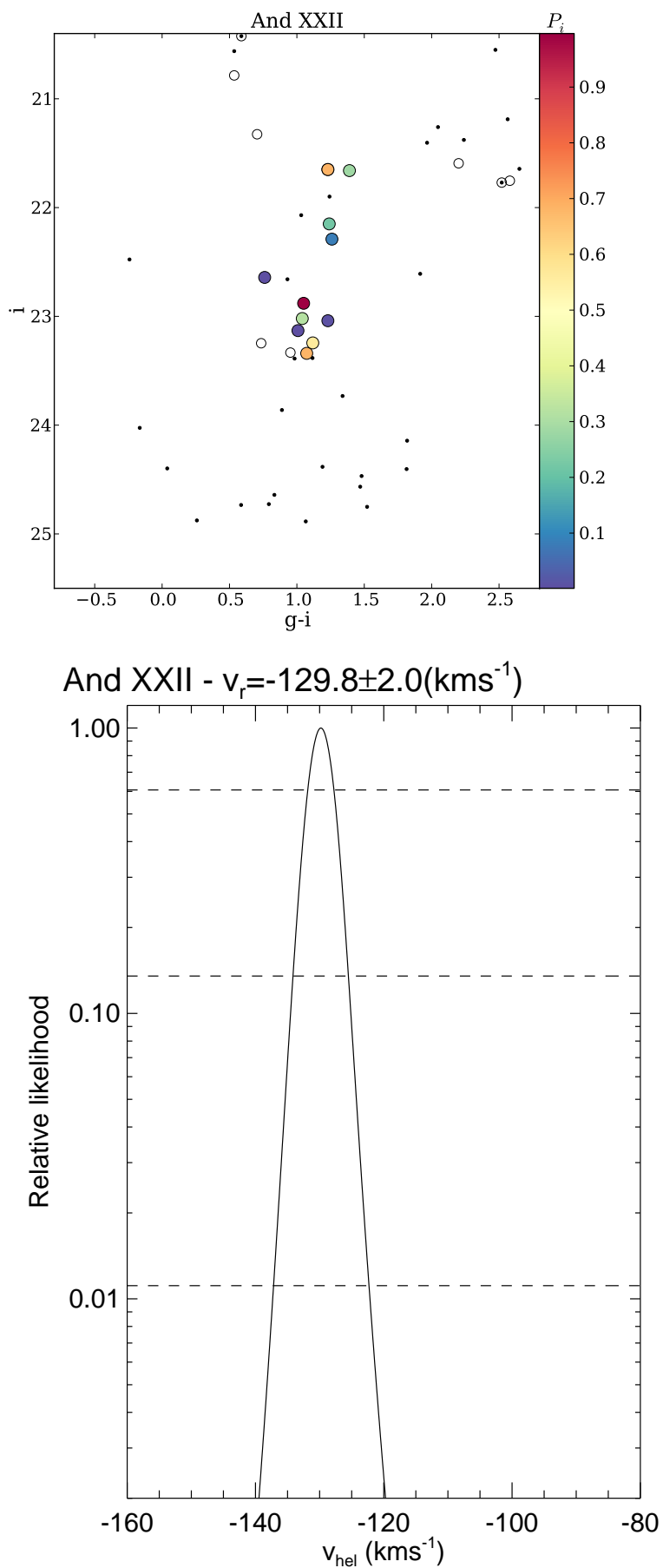

FIG. 11.- As Fig. 6] but for And XXII

and colors that should be consistent with their belonging to And XXVI. However. we find that all these objects have velocities that are consistent with being Galactic foreground contaminants. Given the position of And XXVI in the northern M31 halo, where contamination from the MW increases, this is not unexpected. The brightest star we observe that is likely associated with And XXVI has $m_{i}=21.9\left(m_{i, 0}=21.7\right)$. Assuming that this brightest confirmed member star of And XXVI sits at the RGB tip, the distance estimate becomes 1.1 Mpc.
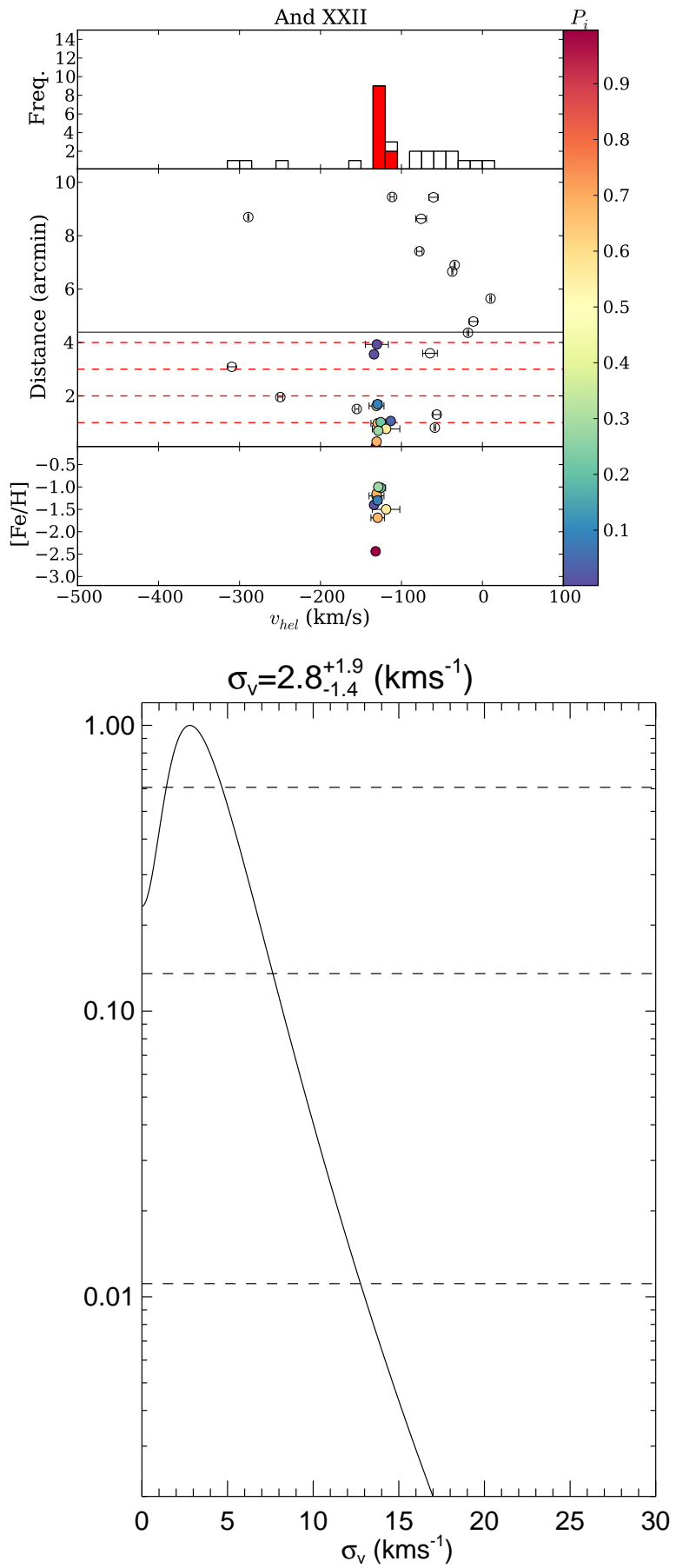

While this value is higher than that of Conn et al. (2011), it is still within their upper distance estimate. Additionally, as not every star brighter than the member with $m_{i, 0}=21.7$, with colors consistent with the And XXVI RGB was observed, this value merely represents an upper limit, on the distance to And XXVI and highlights the difficulty of calculating distances to these faint galaxies where RGB stars are sparse.

\subsection{Andromeda XXVII}



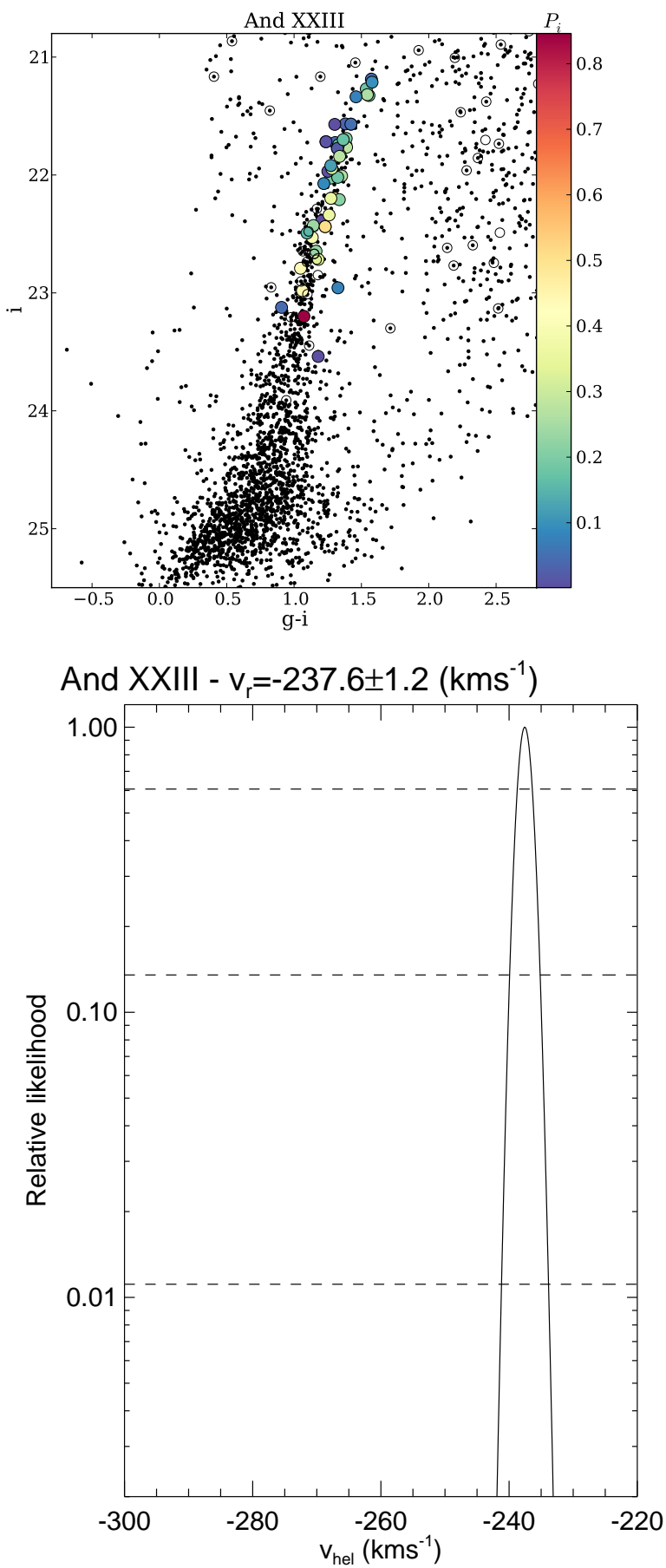
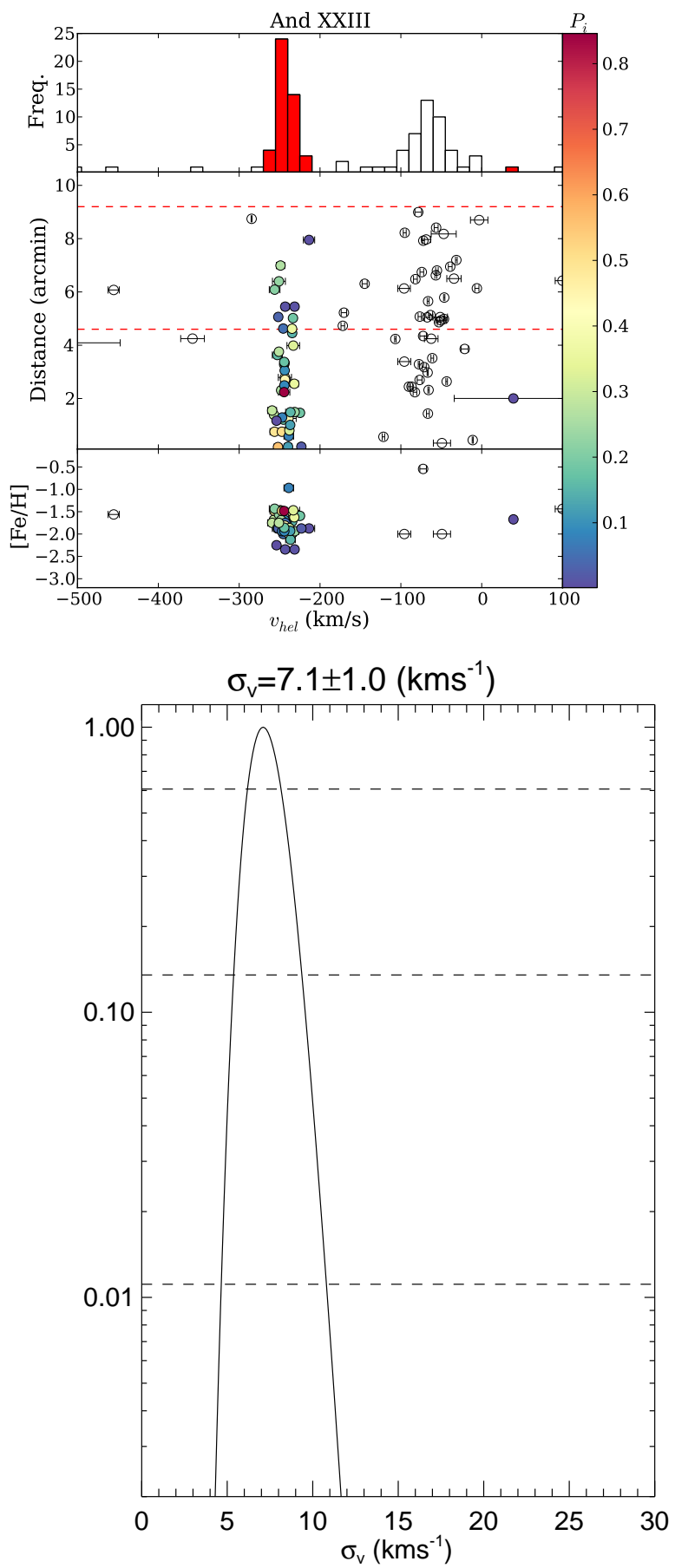

FIG. 12.- As Fig. 6 but for And XXIII.

Andromeda XXVII (And XXVII) is a somewhat unusual object as it is currently undergoing tidal disruption, spreading its constituent stars into a large stellar stream, named the northwestern arc, discovered in the PAndAS survey by Richardson et al. (2011). As such, it is unlikely to be in virial equilibrium, if it remains bound at all.

When determining the kinematics of And XXVII, we find the results somewhat unsatisfactory. Our routine determines $v_{r}=-539.6_{-4.5}^{+4.7} \mathrm{~km} \mathrm{~s}^{-1}$ and $\sigma_{v}=$
$14.8_{-3.1}^{+4.3} \mathrm{~km} \mathrm{~s}^{-1}$ from 11 stars. However, from an inspection of Fig. 16, we see that there is significant substructure around $v_{h e l} \sim-500 \mathrm{~km} \mathrm{~s}^{-1}$, much of which is considered to be unassociated with And XXVII in this analysis as it does not fall within a cold, well-defined Gaussian velocity peak. Given the disrupting nature of And XXVII, it is likely that a different analysis is required for this object, and we shall discuss this further in a future analysis, where the kinematics of the northwestern arc itself are also addressed. From this first pass however, it would ap- 
TABLE 3

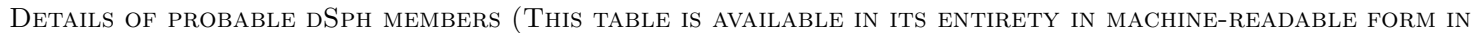
THE ONLINE JOURNAL. A PORTION IS SHOWN HERE FOR GUIDANCE REGARDING ITS FORM AND CONTENT)

\begin{tabular}{lccccccccc}
\hline \hline Field & Star ID & $\alpha_{0, J 2000}$ & $\delta_{0, J 2000}$ & $g$ & $i$ & $v_{h e l}\left(\mathrm{~km} \mathrm{~s}^{-1}\right)$ & $v_{\text {err }}\left(\mathrm{km} \mathrm{s}^{-1}\right)$ & $\mathrm{S}: \mathrm{N}\left(\AA^{-1}\right)$ & $P_{i}$ \\
\hline And V & 9 & $1: 10: 2.38$ & $47: 37: 48.5$ & 23.640 & 22.402 & -371.270 & 5.080 & 1.700 & 0.014 \\
And V & 12 & $1: 10: 5.540$ & $47: 36: 41.6$ & 23.100 & 21.530 & -406.500 & 3.600 & 2.000 & 0.111 \\
$\ldots$ & $\cdots$ & $\cdots$ & $\cdots$ & $\cdots$ & $\cdots$ & $\cdots$ & $\cdots$ & $\cdots$ & $\cdots$ \\
& & & & & & & & $\ldots$ & $\cdots$ \\
\hline
\end{tabular}

pear that And XXVII may no longer be a gravitationally bound system.

\subsection{Andromeda XXVIII}

And XXVIII was recently discovered in the 8th data release of the SDSS survey (Slater et al. 2011). It has $M_{v}=-8.5$ and $r_{\text {half }}=210_{-50}^{+60} \mathrm{pc}$. It is also potentially one of Andromeda's most distant satellites, with a hostsatellite projected separation of $365_{-1}^{+17} \mathrm{kpc}$. The And XXVIII satellite is not covered by the PAndAS footprint, so we must instead use the original SDSS photometry for our analysis. A CMD with the SDSS $i-$ band and $r-i$ colors for And XXVIII is shown in Fig. 17, where all targets brighter that $i \sim 23.5$ within $3 r_{\text {half }}$ are shown. The photometry here do not show an RGB that is as convincing as those from the PAndAS survey, so to guide the eye, we also overplot an isochrone from Dotter et al. (2008) with a metallicity of $[\mathrm{Fe} / \mathrm{H}]=-2.0$, corrected for the distance of And XXVIII as reported in Slater et al. (2011).

In a recent paper, Tollerud et al. (2013) discussed the kinematics of this object as derived from 18 members stars. They find $v_{r}=-328.0 \pm 2.3 \mathrm{~km} \mathrm{~s}^{-1}$ and $\sigma_{v}=$ $8.1 \pm 1.8 \mathrm{~km} \mathrm{~s}^{-1}$ from their full sample. They then remove two stars that they categorize as outliers based on their distance from the centre of And XXVIII, which alters their measurements to $v_{r}=-331.1 \pm 1.8 \mathrm{~km} \mathrm{~s}^{-1}$ and $\sigma_{v}=$ $4.9 \pm 1.6 \mathrm{~km} \mathrm{~s}^{-1}$. Analyzing our own DEIMOS dataset for this object, we find $v_{r}=-326.1 \pm 2.7 \mathrm{~km} \mathrm{~s}^{-1}$ and $\sigma_{v}=6.6_{-2.2}^{+2.9} \mathrm{~km} \mathrm{~s}^{-1}$ based on 17 probable members. This is fully consistent with the results from the full sample in Tollerud et al. (2013). However, the systemic velocity we measure is offset at a level of $\sim 1 \sigma$ from their final value (calculated after excluding 2 outliers). This offset is small, and is probably attributable to our differing methodologies for classifying stars as members. As we believe our method is more robust (as discussed in $\S 3.5$ and Appendix A), we will use our derived parameters for this object in the remainder of our analysis.

\subsection{And XXX/Cassiopeia II}

And XXX - also known as Cass II owing to its spatial location, overlapping the Cassiopeia constellation - is a recently discovered dSph from the PAndAS survey (Irwin et al. in prep). It has $M_{v}=-8.0$ and $r_{\text {half }}=267_{-36}^{+23} \mathrm{pc}$. Located to the north west of Andromeda, it sits within $60 \mathrm{kpc}$ of the two close dwarf elliptical M31 companions, NGC 147 and NGC 185. With these 3 objects found so close together in physical space, it is tempting to suppose them a bound system within their own right, but this can only be borne out by comparing their kinematics.

Conspiring to confound us, we find that Cass II has kinematics that place it well within the regime of Galac- tic foreground, as can be seen in Fig. 18. However, our analysis is able to detect the dSph as a cold spike consisting of 8 likely members. As for And XIX, we check the strength of the Na I doublet in these likely members, and find no significant absorption, making them unlikely foreground contaminants. We measure $v_{r}=-139.8_{-6.6}^{+6.0} \mathrm{~km} \mathrm{~s}^{-1}$, and a fairly typical velocity dispersion of $\sigma_{v}=11.8_{-4.7}^{+7.7} \mathrm{~km} \mathrm{~s}^{-1}$.

The systemic velocity of Cass II $\left(v_{r}=\right.$ $\left.-139.8_{-6.6}^{+6.0} \mathrm{~km} \mathrm{~s}^{-1}\right)$ puts it within $\sim 50 \mathrm{~km} \mathrm{~s}^{-1}$ of those of NGC 147 and NGC $185\left(v_{r}=-193 \pm 3 \mathrm{~km} \mathrm{~s}^{-1}\right.$ and $v_{r}=-210 \pm 7 \mathrm{~km} \mathrm{~s}^{-1}$, Mateo 1998), lending further credence to the notion that these 3 systems are associated with one another. This will be discussed in more detail in Irwin et al. (2013, in prep).

\subsection{A note on previous work}

Finally, we also use our new algorithm to reanalyze all our previously published M31 dSph datasets. These include And V, VI (Collins et al. 2011), XI, XII and XIII (Chapman et al. 2007; Collins et al. 2010). Details of the results of this reanalysis can be found in Appendix B. In summary, we find that our algorithm measures systemic velocities and velocity dispersions that are fully consistent with our previous work. We present these results in Table 4. And V, XI, XII and XIII are also analyzed by T12, so we compare our findings with theirs. For And XI and XII, our results are based on two and four times the number of stars respectively, and as such, supercede those presented in T12. In the case of And V and XIII, the T12 measurements are based sample sizes with four times the number of stars as our datasets, making their findings more robust.

In previous studies by our group (Chapman et al. 2005; Letarte et al. 2009; Collins et al. 2010), we also published kinematic analyses for three additional M31 dSphs; And IX, And XV and And XVI. In T12, it was noted that the values presented in these works for systemic velocities and velocity dispersions were not consistent with those measured in their analyses. We revisited these datasets in light of this discrepancy, to see if our new technique could resolve this issue. We found that these discrepancies remained. For And IX, we measure a systemic velocity of $v_{r}=-204.8 \pm 2.1 \mathrm{~km} \mathrm{~s}^{-1} \mathrm{cf}$. $v_{r}=-209.4 \pm 2.5 \mathrm{~km} \mathrm{~s}^{-1}$ in T12 and a velocity dispersion of $\sigma_{v}=2.0_{-2.0}^{+2.7} \mathrm{~km} \mathrm{~s}^{-1}$ cf. $\sigma_{v}=10.9 \pm 2.0 \mathrm{~km} \mathrm{~s}^{-1}$. Not only are their measurements determined from 4 times the member stars that we possess, we also experienced problems with our radial velocity measurements for the stars observed with this mask, due to the use of the minislitlet approach pioneered by Ibata et al. (2005). This setup resulted in poor sky subtraction for many of the science 

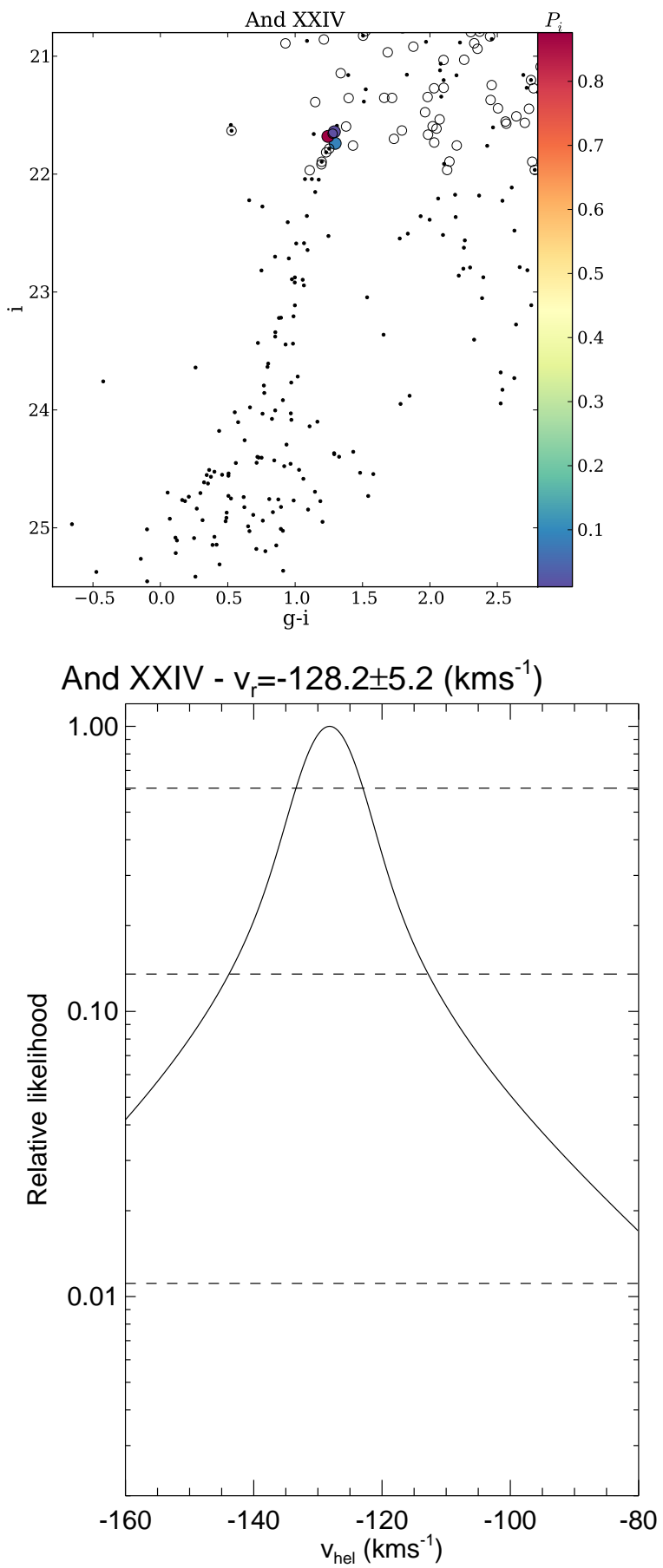
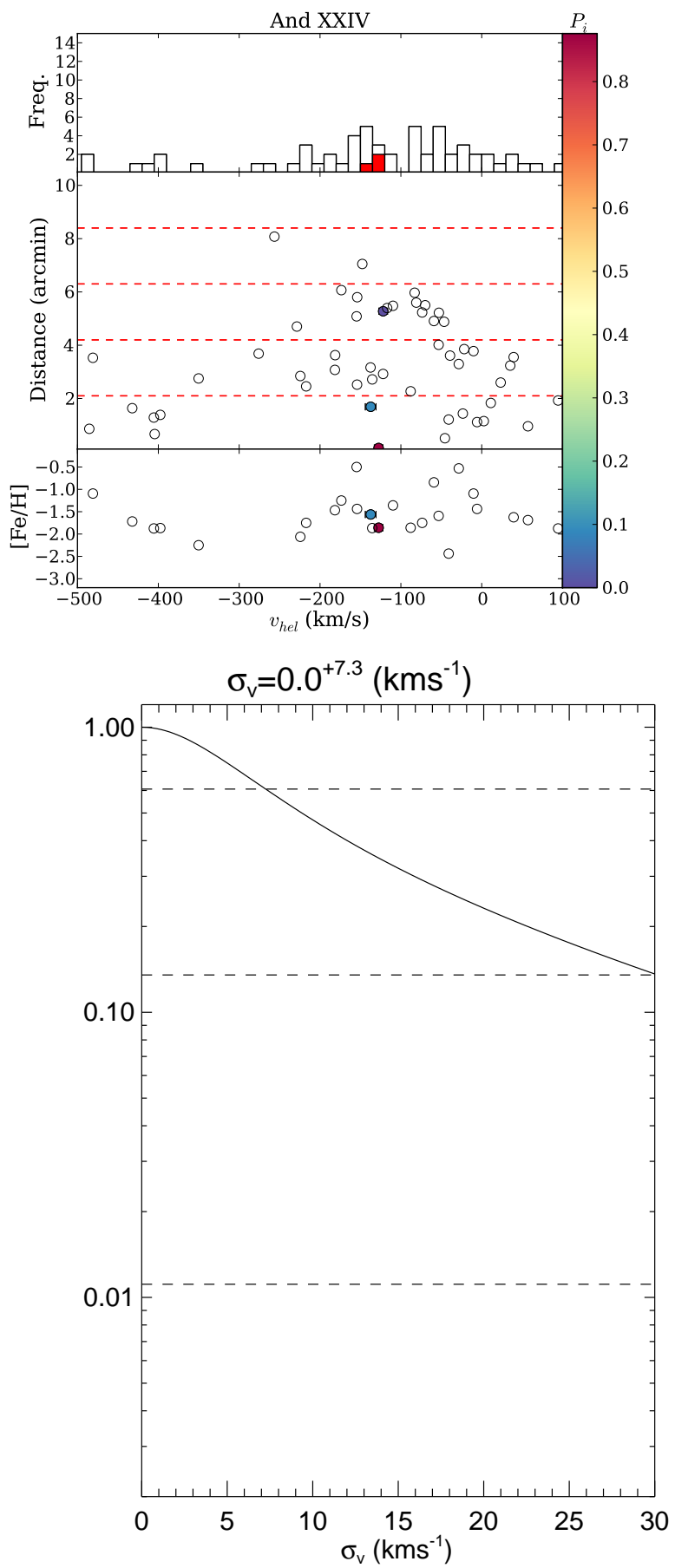

FIG. 13.- As Fig. 6 but for And XXIV.

spectra, lowering the quality of our radial velocity measurements. As such, the T12 results supercede those of our previous work (Chapman et al. 2005; Collins et al. 2010).

For And XV and XVI, we measure a systemic velocities of $v_{r}=-354.6 \pm 4.9 \mathrm{~km} \mathrm{~s}^{-1}$ and $v_{r}=-374.1 \pm 6.8 \mathrm{~km} \mathrm{~s}^{-1}$ cf. $\quad v_{r}=-323.0 \pm 1.4 \mathrm{~km} \mathrm{~s}^{-1}$ and $v_{r}=-367.3 \pm$ $2.8 \mathrm{~km} \mathrm{~s}^{-1}$ from T12. We also note offsets in our velocity dispersions for And XV and XVI, where we measure $\sigma_{v}=9.6_{-2.6}^{+4.1} \mathrm{~km} \mathrm{~s}^{-1}$ and $\sigma_{v}=17.3_{-4.4}^{+6.4} \mathrm{~km} \mathrm{~s}^{-1} \mathrm{cf}$. $\sigma_{v}=3.8 \pm 2.9 \mathrm{~km} \mathrm{~s}^{-1}$. In this instance, the data for both And XV and XVI were taken in poor conditions, with variable seeing that averaged at $1.8^{\prime \prime}$ and patchy cirrus. These conditions significantly deteriorated the quality of our spectra, and made the measurement of reliable radial velocities extremely difficult. Again, this leads us to conclude that the measurements made in T12 supercede those presented by our group in Letarte et al. (2009). 

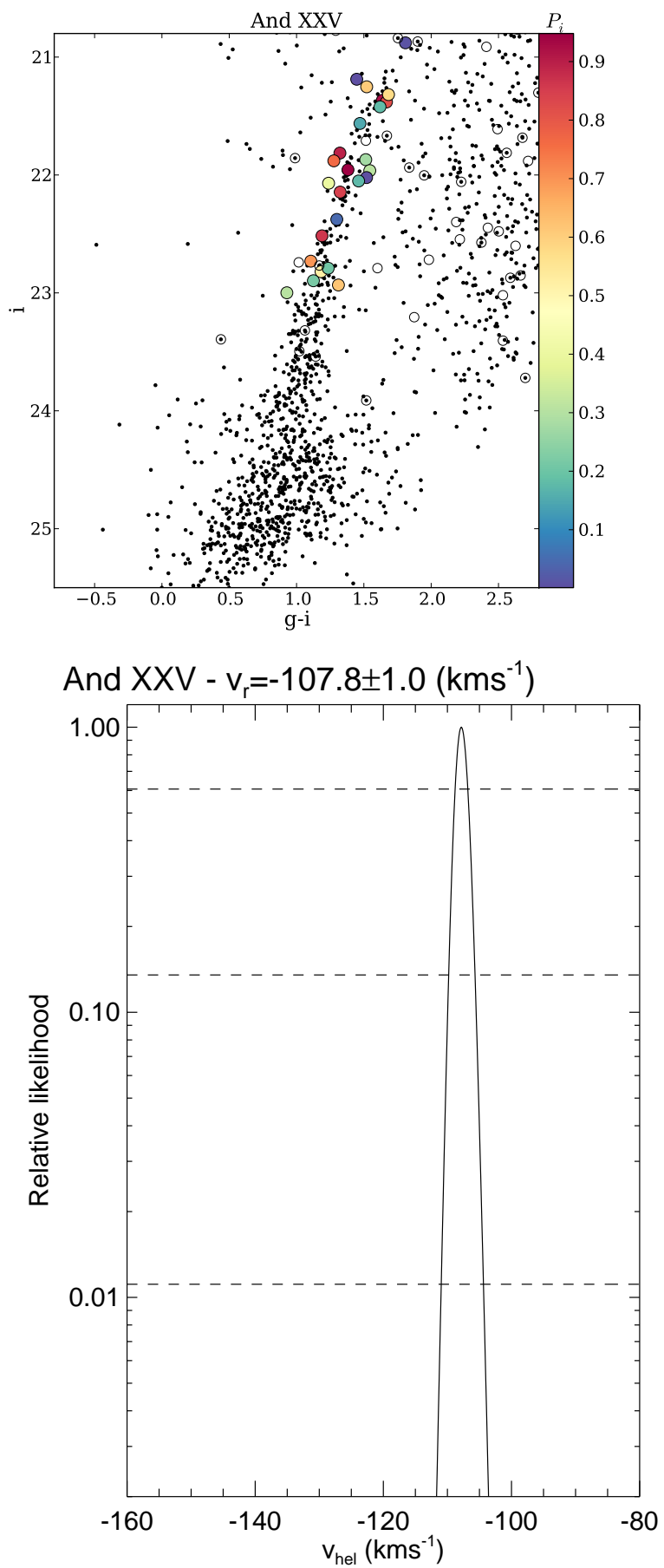
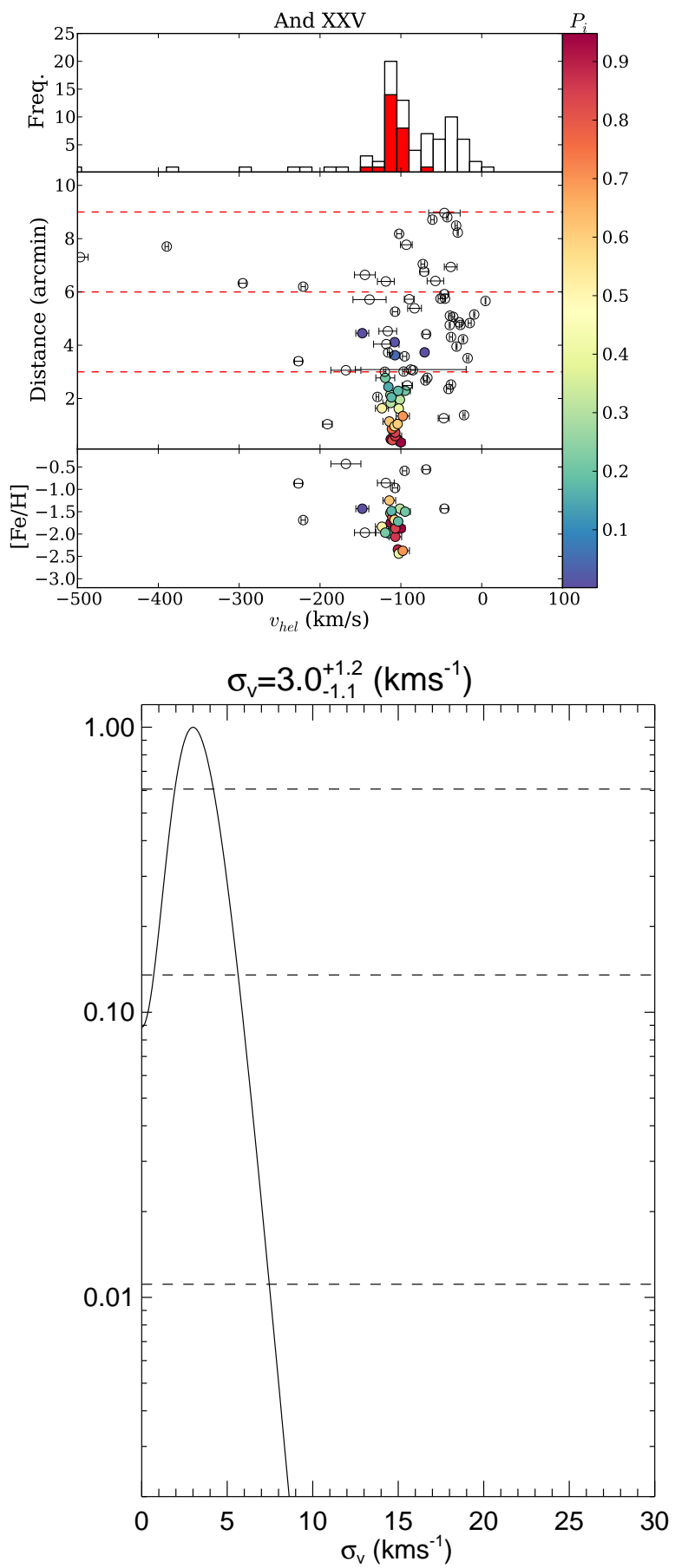

FIG. 14.- As Fig. [6 but for And XXV.

\section{THE MASSES AND DARK MATTER CONTENT OF M31} DSPHS

\subsection{Measuring the masses and mass-to-light ratios of our sample}

As dSph galaxies are predominantly dispersion supported systems, we can use their internal velocity dispersions to measure masses for these systems, allowing us to infer how dark matter dominated they are. There are several methods in the literature for this (e.g., Illingworth
1976; Richstone \& Tremaine 1986), but recent work by Walker et al. (2009a) has shown that the mass contained within the half-light radius $\left(M_{\text {half }}\right)$ of these objects can be reliably estimated using the following formula:

$$
M_{\text {half }}=\mu r_{\text {half }} \sigma_{v, \text { half }}^{2}
$$

where $\mu=580 \mathrm{M}_{\odot} \mathrm{pc}^{-1} \mathrm{~km}^{-2} \mathrm{~s}^{2}, r_{\text {half }}$ is the spherical halflight radius in pc and $\sigma_{v}$, half is the luminosity- averaged velocity dispersion. This mass estimator is independent 

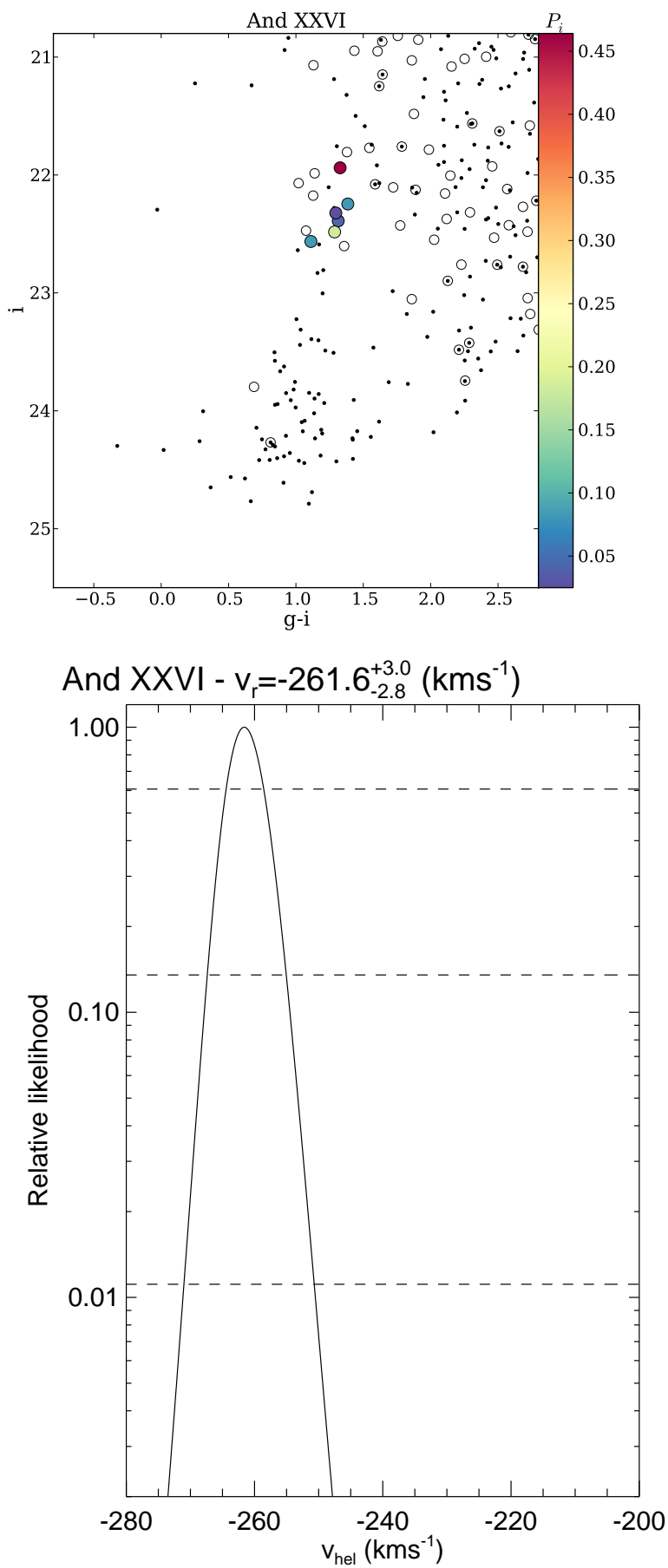
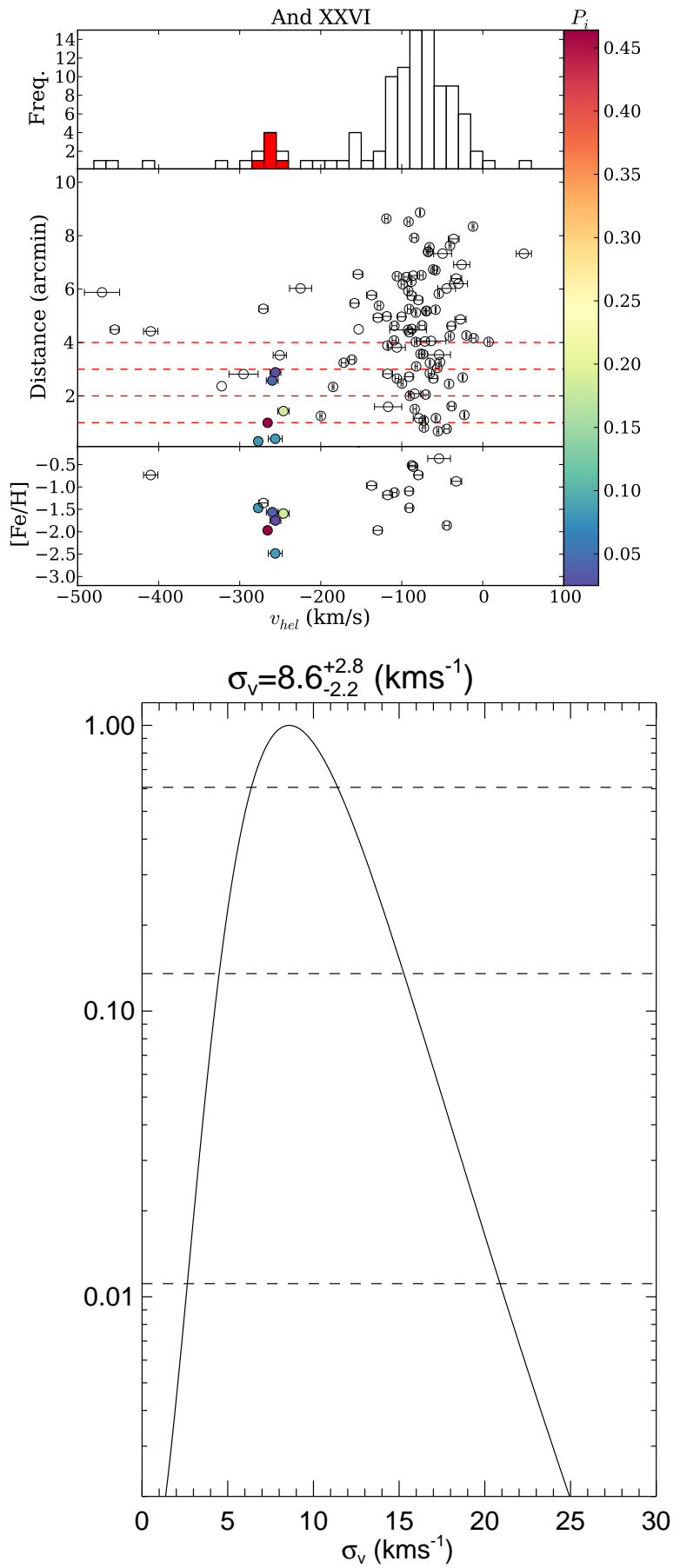

FIG. 15.- As Fig. 6 but for And XXVI.

of the (unknown) velocity anisotropy of the tracer population, however, it is sensitive to the embeddedness of the stellar component within the DM halo. Particularly, the mass tends to be slightly over-estimated the more embedded the stars are (Walker \& Peñarrubia 2011), especially if the dark matter halo follows a cored density profile.

As numerous authors have shown that the velocity dispersion profiles of dSphs are constant with radius (e.g., Walker et al. 2007, 2009b), we assume our measured values of $\sigma_{v}$ are representative of the luminosity-averaged velocity dispersion $\left(\sigma_{v}\right.$,half $)$ used by Walker et al. (2009b). However, if it transpired that the velocity dispersion profiles of the Andromedean dSphs were not flat, but declined or increased with radius, this would no longer true. We see no evidence for this behaviour in our dataset, although low-number statistics means we are unable to completely rule out this possibility. We calculate this for all our observed dSphs (including those we reanalyzed from previous works, see 

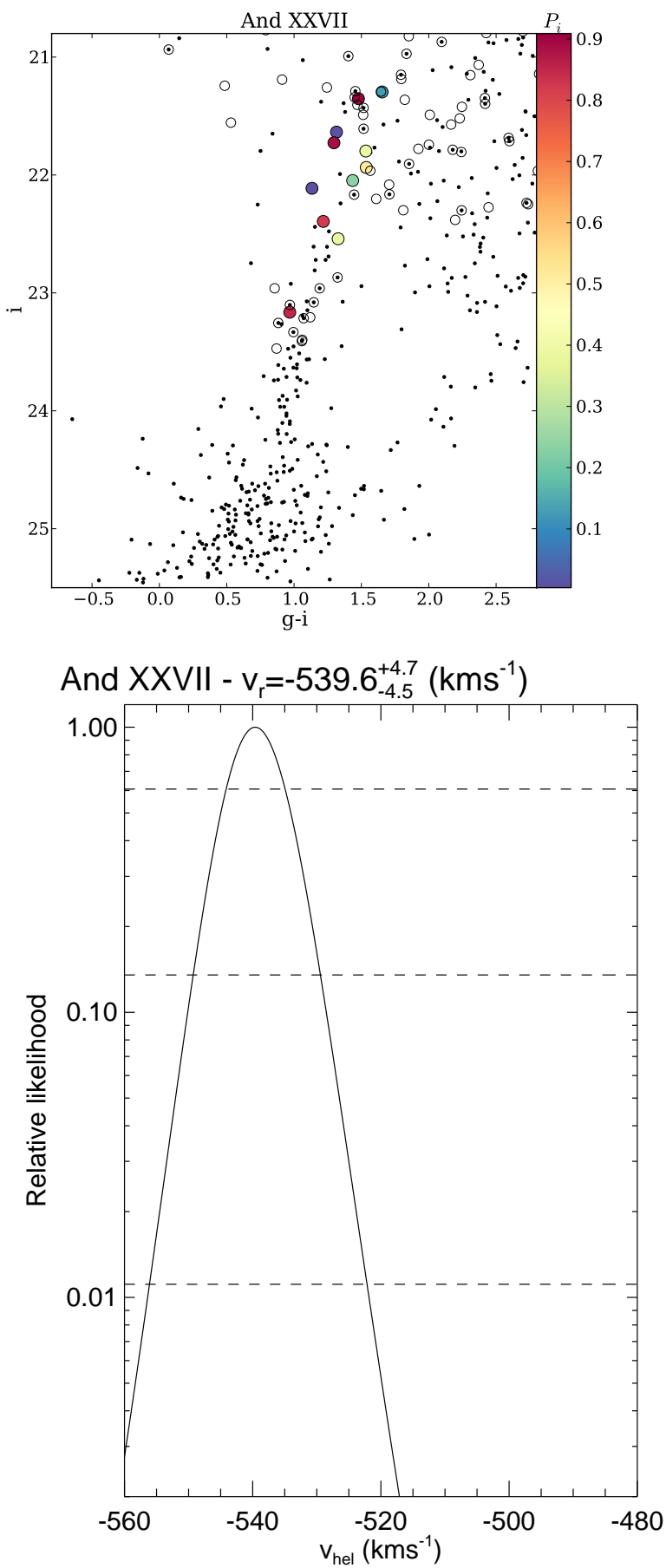

FIG. 16.- As Fig. 6 but for And XXVII.

Appendix B) using results from the Keck LRIS and DEIMOS dataset, and report their masses within $r_{\text {half }}$ $\left(M_{\text {half }}\right)$ in Table 4 .

From these masses, it is trivial to estimate the dynamical central mass-to-light ratios for the objects, $[M / L]_{\text {half }}$. We list these values for each dSph in Table 4, where the associated uncertainties also take into account those from the measured luminosities and distances to these dSphs (McConnachie 2012; Conn et al. 2012), as well as those on the masses measured in this work.
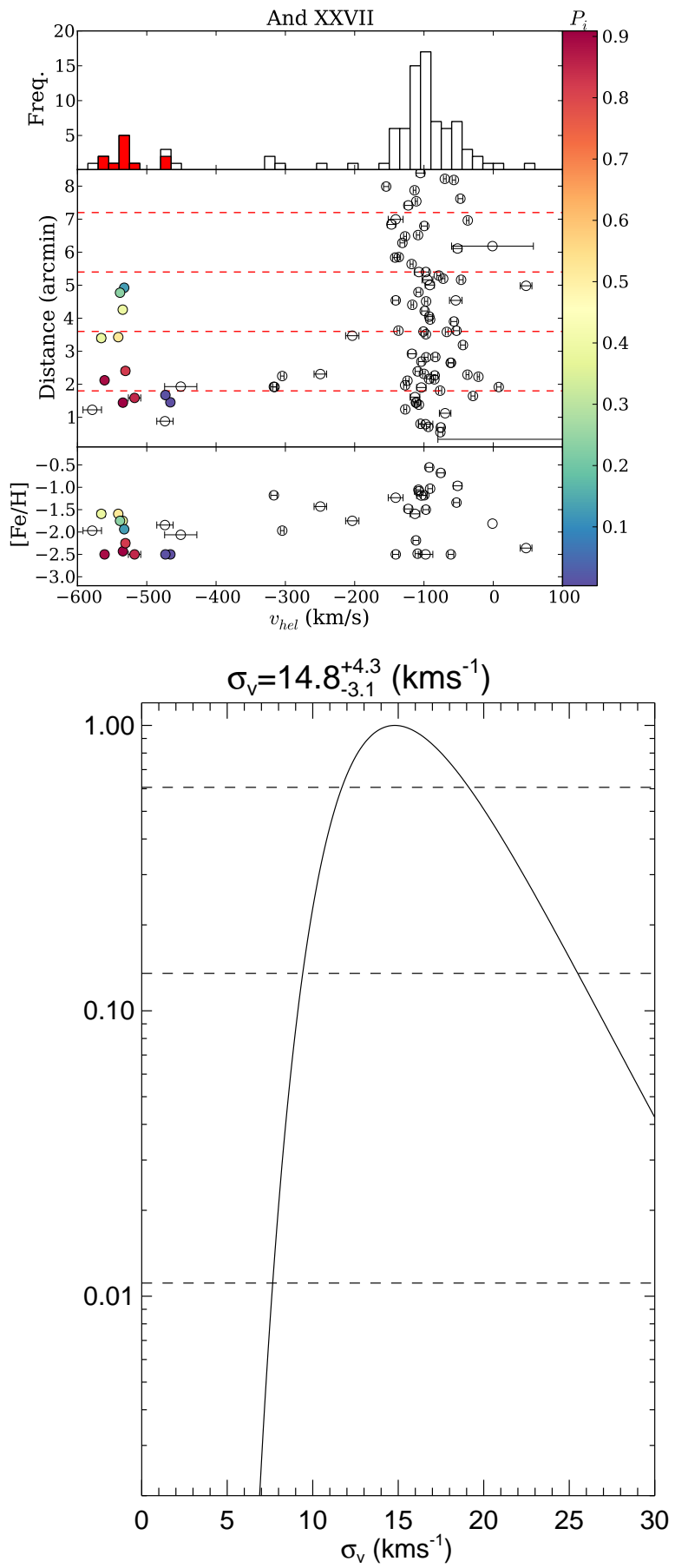

\subsection{Comparing the mass-to-light ratios of M31 and Milky Way dSphs}

By combining our measurements of the kinematic of M31 dSphs in this work with those from T12 and Tollerud et al. (2013), we find ourselves with a set of kinematic properties as measured for 27 of the 28 Andromeda dSphs (owing to the difficulties experienced with the And XXIV dataset, we do not include this object in our subsequent analysis). This near-complete 

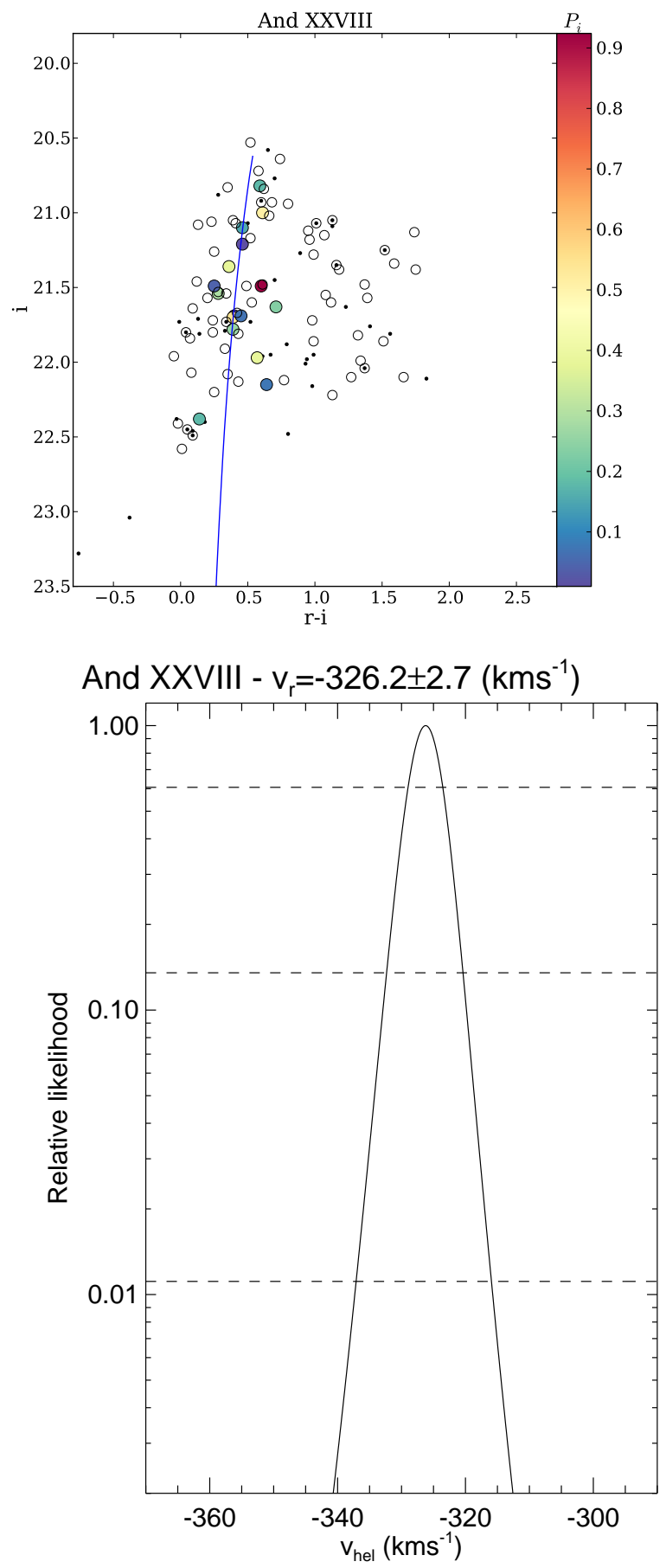

FIG. 17.- As Fig. 6 but for And XXVIII.

sample allows us to fully compare the masses and massto-light ratios for the M31 satellite system with those measured in the Milky Way satellites. Before beginning this analysis, we compile Table 5 which presents the kinematics for the full M31 satellite system, which combines the results from this work, T12, Kalirai et al. (2010), and Tollerud et al. (2013). In cases where two measurements for a dSph exist, we use those that were calculated from larger numbers of likely members, as these are the more robust. We begin by comparing the mass-to-light ra-
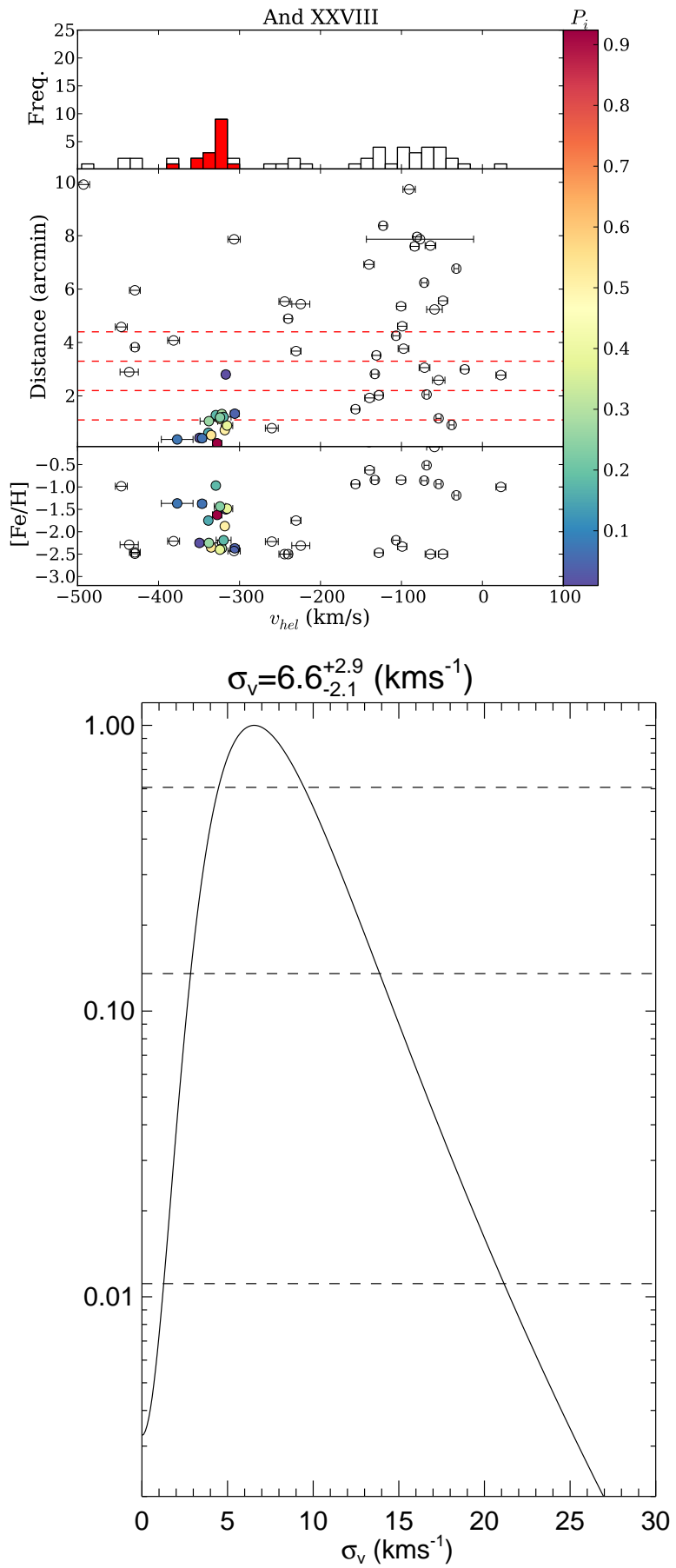

tios (which indicate the relative dark matter dominance of these objects) of the two populations as a function of luminosity. In Fig 19 we show these values for all MW (red triangles, with values taken from Walker et al. 2009b), and M31 (blue circles) dSphs as a function of their luminosity. We can see that all these objects are clearly dark matter dominated, excluding And XII and And XVII where we are unable to resolve the mass with current datasets. We also see that they follow the trend of increasing $[M / L]_{\text {half }}$ with decreasing luminosity, as is 

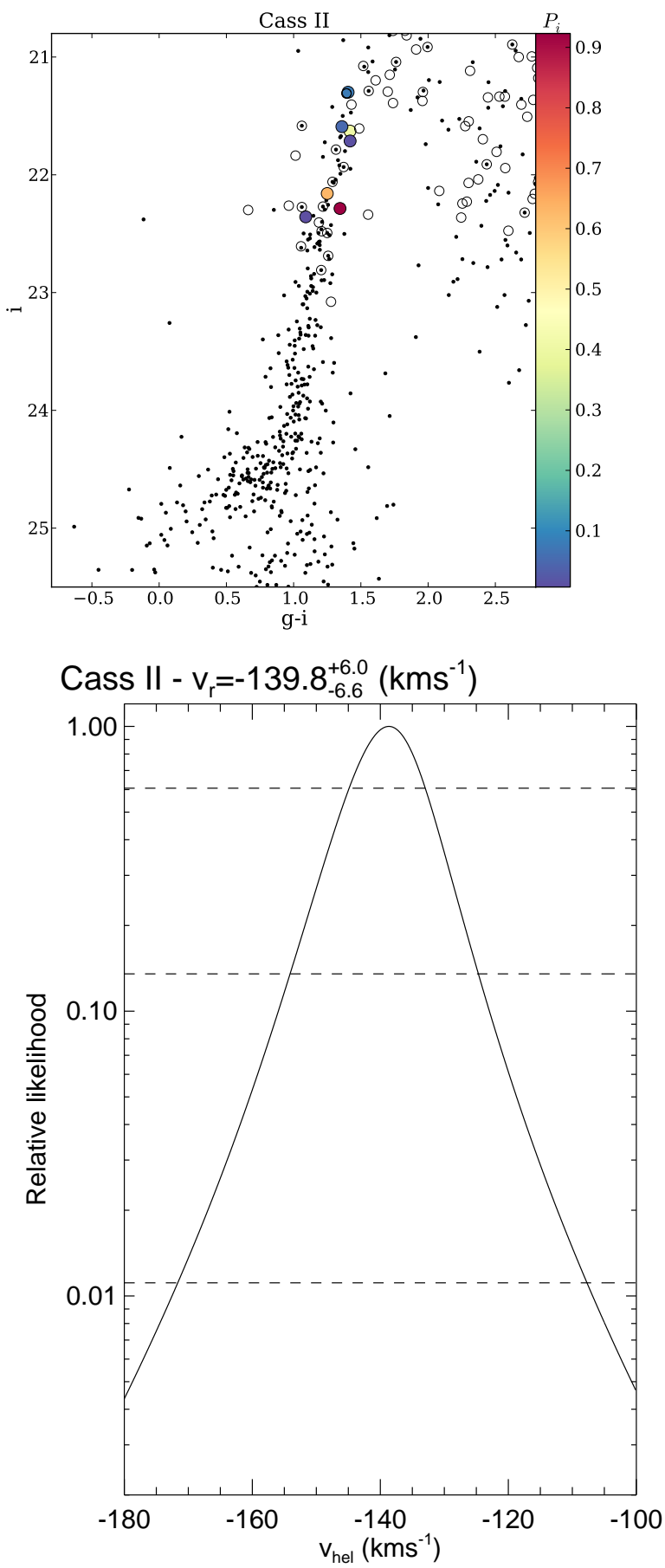
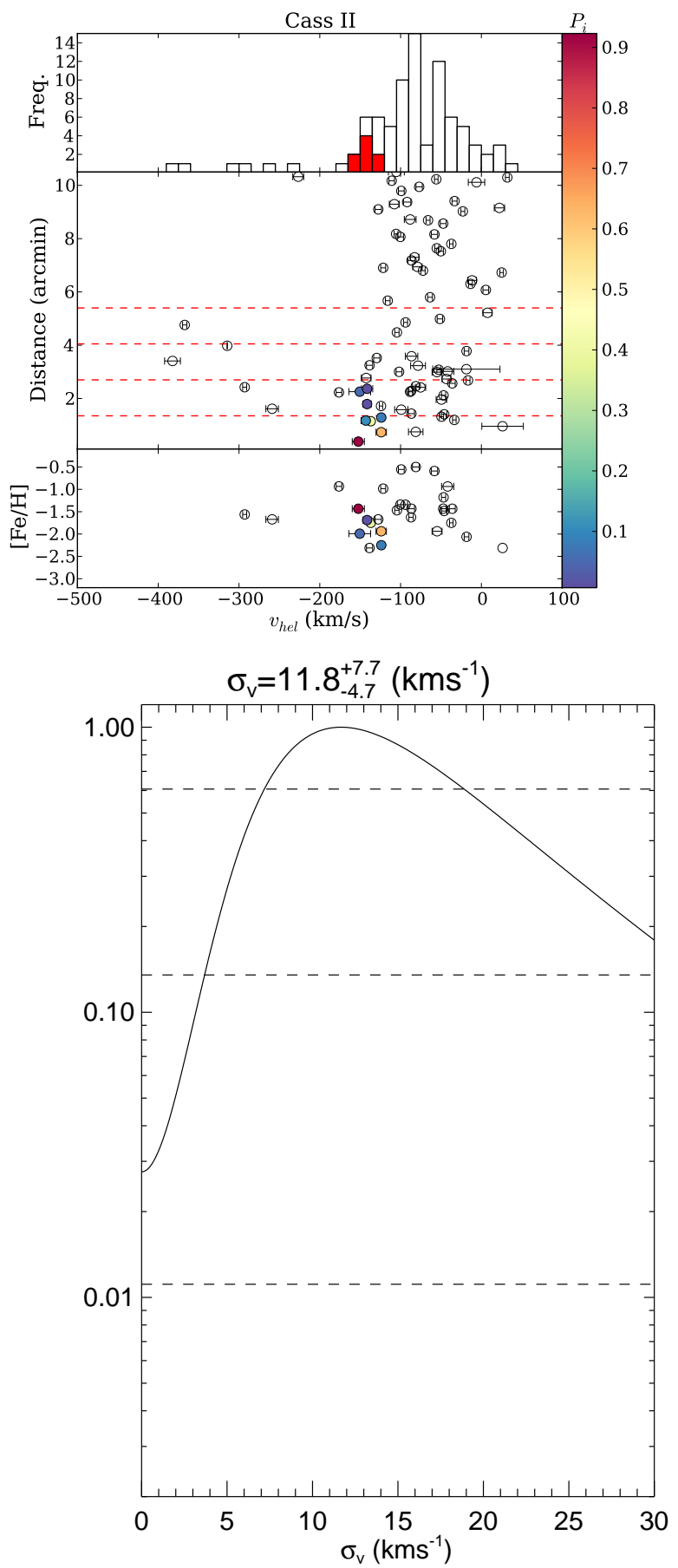

FIG. 18.- As Fig. 6] but for Cass II

seen in their MW counterparts.

The one tentative exception to this is the And XXV dSph. From our dataset, we measure a value of $[M / L]_{\text {half }}=10.3_{-6.7}^{+7.0}$ for this object, making it consistent with a stellar population with no dark matter within its $1 \sigma$ uncertainties. This result is surprising and would be of enormous importance if confirmed with a larger dataset than our catalog of 26 likely members as it would be the first $\mathrm{dSph}$ to be observed with a negligible dark matter component. And XXV is also one of the mem- bers of the recently discovered thin plane of satellites in Andromeda (Ibata et al. 2013), and so the presence or absence of dark matter in And XXV might tell us more about the origins of this plane which are currently poorly understood.

\subsection{Comparing the masses of M31 and Milky Way} dSphs

Finally, we discuss how the masses for the full sample of Andromeda dSphs for which kinematic data are 
TABLE 4

Kinematic Properties of ANDromeda dSph galaxies as DeRIVEd Within this WORK, FROM KECK I LRIS AND KECK II DEIMOS DATA.

\begin{tabular}{lcccccc}
\hline \hline \multicolumn{1}{c}{ Property } & $\eta$ & $\begin{array}{c}v_{r} \\
\left(\mathrm{~km} \mathrm{~s}^{-1}\right)\end{array}$ & $\begin{array}{c}\sigma_{v} \\
\left(\mathrm{~km} \mathrm{~s}^{-1}\right)\end{array}$ & $\begin{array}{c}\mathrm{M}_{\text {half }} \\
\left(10^{7} \mathrm{M}_{\odot}\right)\end{array}$ & $\begin{array}{c}{[M / L]_{\text {half }}} \\
\left(\mathrm{M}_{\odot} / \mathrm{L}_{\odot}\right)\end{array}$ & {$[\mathrm{Fe} / \mathrm{H}]_{\text {spec }}$} \\
\hline And V & 2.0 & $-391.5 \pm 2.7$ & $12.2_{-1.9}^{+2.5}$ & $2.6_{-0.56}^{+0.66}$ & $88.4_{-18.9}^{+22.3}$ & $-2.0 \pm 0.1$ \\
And VI & 2.5 & $-339.8 \pm 1.8$ & $12.4_{-1.3}^{+1.5}$ & $4.7 \pm 0.7$ & $27.5_{-3.9}^{+4.2}$ & $-1.5 \pm 0.1$ \\
And XI & 2.5 & $-427.5_{-3.4}^{+3.5}$ & $7.6_{-2.8}^{+4.0(*)}$ & $0.53_{-0.21}^{+0.28}$ & $216_{-87}^{+115}$ & $-1.8 \pm 0.1$ \\
And XII & 2.5 & $-557.1 \pm 1.7$ & $0.0^{+4.0}$ & $0.0^{+0.3}$ & $0.0^{+194}$ & $-2.2 \pm 0.2$ \\
And XIII & 2.5 & $-204.8 \pm 4.9$ & $0.0^{+8.1(*)}$ & $0.0^{+0.7}$ & $0.0^{+330}$ & $-1.7 \pm 0.3$ \\
And XVII & 2.5 & $-251.6_{-2.0}^{+1.8}$ & $2.9_{-1.9}^{+2.2}$ & $0.13_{-0.13}^{+0.22}$ & $12_{-12}^{+22}$ & $-1.7 \pm 0.2$ \\
And XVIII & 2.5 & $-346.8 \pm 2.0$ & $0.0^{+2.7}$ & $0.0^{+0.14}$ & $0^{+5}$ & $-1.4 \pm 0.3$ \\
And XIX & 2.0 & $-111.6_{-1.4}^{+1.6}$ & $4.7_{-1.4}^{+1.6}$ & $1.9_{-0.65}^{+0.65}$ & $84.3_{-38}^{+37}$ & $-1.8 \pm 0.3$ \\
And XX & 2.5 & $-456.2_{-3.6}^{+3.1}$ & $\left.7.1_{-2.5}^{+3.9}\right)$ & $0.33_{-0.12}^{+0.20}$ & $238.1_{-90.2}^{+147.6}$ & $-2.2 \pm 0.4$ \\
And XXI & 5.0 & $-362.5 \pm 0.9$ & $4.5_{-1.0}^{+1.2}$ & $0.99_{-0.24}^{+0.28}$ & $25.4_{-8.4}^{+9.4}$ & $-1.8 \pm 0.1$ \\
And XXII & 2.0 & $-129.8 \pm 2.0$ & $2.8_{-1.4}^{+1.9}$ & $0.11_{-0.06}^{+0.08}$ & $76.4_{-48.1}^{+58.4}$ & $-1.8 \pm 0.6$ \\
And XXIII & 4.0 & $-237.7 \pm 1.2$ & $7.1 \pm 1.0$ & $2.9 \pm .4 .4$ & $58.5 \pm 36.2$ & $-2.2 \pm 0.3$ \\
And XXIV & 1.5 & $-128.2 \pm 5.2$ & $0.0^{+7.3(*)}$ & $0.4_{-0.4}^{+0.7}$ & $82_{-82}^{+157}$ & $-1.8 \pm 0.3$ \\
And XXV & 2.5 & $-107.8 \pm 1.0$ & $3.0_{-1.1}^{+1.2}$ & $0.34_{-0.14}^{+0.14}$ & $10.3_{-6.7}^{+7.0}$ & $-1.9 \pm 0.1$ \\
And XXVI & 3.0 & $-261.6_{-2.8}^{+3.0}$ & $8.6_{-2.2}^{+2.8}$ & $0.96_{-0.34}^{+0.43}$ & $325_{-225}^{+243}$ & $-1.8 \pm 0.5$ \\
And XXVII & 1.5 & $-539.6_{-4.5}^{+4.7}$ & $14.8_{-3.1}^{+4.3}$ & $8.3_{-3.9}^{+2.8}$ & $1391_{-1128}^{+1039}$ & $-2.1 \pm 0.5$ \\
And XXVIII & 2.5 & $-326.2 \pm 2.7$ & $6.6_{-2.1}^{+2.9}$ & $0.53_{-0.21}^{+0.28}$ & $51_{-25}^{+30}$ & $-2.1 \pm 0.3$ \\
And XXX (Cass II) & 2.0 & $-139.8_{-6.6}^{+6.0}$ & $11.8_{-4.7}^{+7.7}$ & $2.2_{-0.9}^{+1.4}$ & $308_{-219}^{+269}$ & $-1.7 \pm 0.4$ \\
& & & & & &
\end{tabular}

Note. - ${ }^{(*)}$ - indicates velocity dispersions derived from fewer than 8 members stars, and require confirmation from further follow-up.

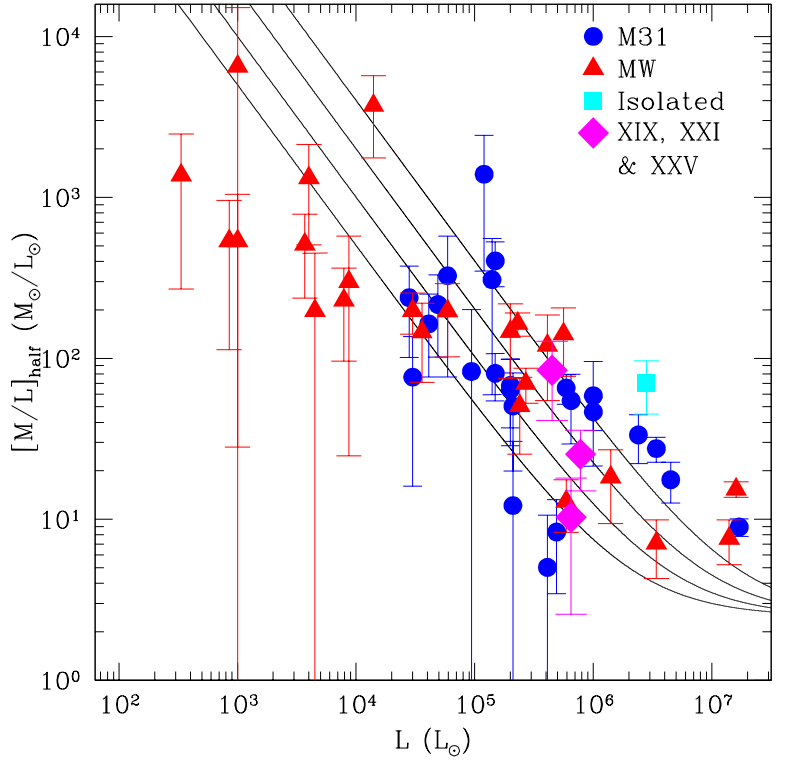

FIG. 19.- Dynamical mass-to-light ratio within the half-light radius, $[M / L]_{\text {half }}$, as a function of half-light radius for all M31 (blue circles), MW (red triangles) and isolated dSphs (cyan squares).

available compare with those of the MW dSphs. For the M31 dSph population, we again use our compilation of kinematic properties assembled in Table 5. We plot the velocity dispersions, mass within the half-light radius, and central densities for all M31 (blue circles) and MW (red triangles, Walker et al. 2009b; Adén et al. 2009; Koposov et al. 2011; Simon et al. 2011) dSphs as a function of radius. We then overplot the best-fit NFW and cored mass profiles for the MW, taken from Walker et al. (2009b). In general, we see that the M31 and MW objects are similarly consistent with these profiles, an agreement that was also noted by T12. However, there are 3 objects which are clear outliers to these relations. These are And XIX, XXI and XXV, with velocity dispersions of $\sigma_{v}=4.7_{-1.4}^{+1.6} \mathrm{~km} \mathrm{~s}^{-1}, \sigma_{v}=4.5_{-1.0}^{+1.2} \mathrm{~km} \mathrm{~s}^{-1}$ and $\sigma_{v}=3.0_{-1.1}^{+1.2} \mathrm{~km} \mathrm{~s}^{-1}$, as derived in this work. Given their half-light radii $\left(r_{\text {half }}=1481_{-268}^{+62} \mathrm{pc}, r_{\text {half }}=842 \pm 77 \mathrm{pc}\right.$ and $r_{\text {half }}=642_{-74}^{+47} \mathrm{pc}$ ), one would expect them to have dispersions of closer to $9 \mathrm{~km} \mathrm{~s}^{-1}$ in order to be consistent with the MW mass profile. As they stand, these three objects are outliers at a statistical significance of $2.5 \sigma$, $3.0 \sigma$ and $3.4 \sigma$ (calculated directly from their likelihood distributions as presented in Figs. 8 , 10 and 14). Similarly, in T12 they noted that And XXII and And XIV were outliers in the same respect as And XIX, XXI and XXV, albeit at a lower significance. These difference can also be observed in terms of the enclosed masses and densities within $r_{\text {half }}$.

In Collins et al. (2011) we argued that the low velocity dispersion seen in some Andromeda dwarfs were a result of tidal forces exerted on their halos by the host over the course of their evolution, and that this effect was predominantly seen in dSphs where their half-light radii were more extended for a given luminosity than expected, such is the case our three outliers, And XIX, XXI and XXV. This result therefore adds weight to the trend presented in that work. A number of recent works trying to account for the lower than predicted central masses of dSph galaxies within the Local Group also support this notion. For example, Peñarrubia et al. (2010) demonstrated that the presence of a massive stellar disk in the host galaxy (such as those of the MW and M31) can significantly reduce the total masses of its associated satel- 


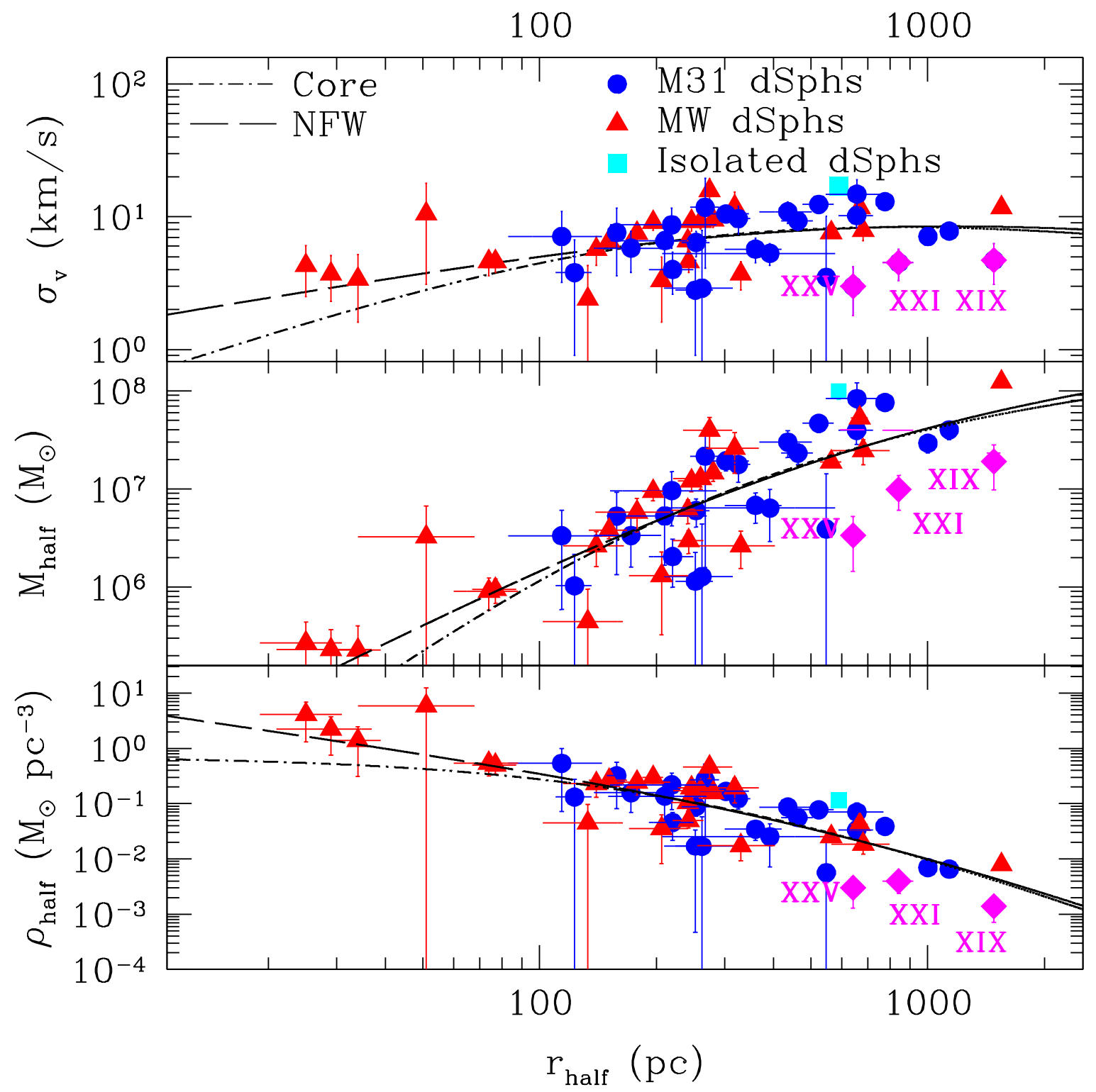

FIG. 20.- Top: Half-light radius ( $\left.r_{\text {half }}\right)$ vs. velocity dispersion for the MW (red triangles) and M31 (blue circles) dSphs. The best fit NFW and cored mass profiles to the whole Local Group dSph population are over-plotted as dashed and dot-dashed lines respectively. Middle: As above, but in the $M_{\text {half }}-r_{\text {half }}$ plane. Lower: As above but in the $\rho_{\text {half }}-r_{\text {half }}$ plane.

lites. In addition, recent, papers by Zolotov et al. (2012) and Brooks \& Zolotov (2012), where the effect of baryons within dark matter only simulations was measured also find that tidal forces exerted by host galaxies where a massive disk is present will serve to reduce the masses of its satellite population at a far greater rate than hosts without baryons. And XIX, XXI and XXV may thus represent a population of dSph satellites whose orbital histories about M31 have resulted in substantial fractions of their central mass being removed by tides. It should be noted, however, that tides not only reduce the central masses and densities of dSph halos, they also reduce the spatial size of the luminous component (Peñarrubia et al. 2008b, 2010), albeit at a slower rate. The tidal scenario is therefore slightly difficult to reconcile with these outlying M31 dSphs having the largest sizes, unless they were both more massive and spatially larger in the past.

Other recent theoretical works have also shown that the removal of baryons from the very centres of dark matter halos by baryonic feedback (from star formation and supernovae, for example) can also help to lower the central masses and densities of satellite galaxies (e.g., Pontzen \& Governato 2012; Zolotov et al.|2012; Brooks \& Zolotov 2012). For this method to work effectively, however, very large 'blow outs' of gas are required, of the order $\sim 10^{8}-10^{9} \mathrm{M}_{\odot}$, equivalent to $\sim 40000 \mathrm{SNe}$. This would require a minimum initial satellite luminosity of $M_{V}<-12$ (Zolotov et al. 2012; Garrison-Kimmel et al. 2013), significantly brighter than the current luminosities of our outliers $\left(M_{V} \sim-10\right)$. Therefore, if feedback has indeed played a role in the shaping of the dark matter halos of And XIX, XXI and 
XXV, one assumes it would have to have operated in tandem with tidal stripping. We will discuss the implications and interpretation of these result further in a companion paper (Collins et al. in prep).

\section{METALLICITIES}

Our observational setup was such that we cover the calcium triplet region (Ca II) of all our observed stars. This strong, absorption feature is useful not only for calculating velocities for each star, but also metallicities. For RGB stars, such as we have observed, there is a well known relation between the equivalent widths (EWs) of the $\mathrm{Ca}$ II lines, and the iron abundance, $[\mathrm{Fe} / \mathrm{H}]$, of the object. The calibration between these two values has been studied and tested by numerous authors, using both globular clusters and dSphs, and is valid down to metallicities as low as $[\mathrm{Fe} / \mathrm{H}] \sim-4$ (see e.g., Battaglia et al. 2008; Starkenburg et al. 2010). Following the Starkenburg et al. (2010) method, which extends the sensitivity of this method down to as low as $[\mathrm{Fe} / \mathrm{H}] \sim-4$, we fit Gaussian functions to the three $\mathrm{Ca}$ II peaks to estimate their equivalent widths (EWs), and calculate $[\mathrm{Fe} / \mathrm{H}]$ using equation 13 .

$$
\begin{array}{r}
{[\mathrm{Fe} / \mathrm{H}]=-2.87+0.195\left(V_{R G B}-V_{H B}\right)+0.48 \Sigma \mathrm{Ca}} \\
-0.913 \Sigma \mathrm{Ca}^{-1.5}+0.0155 \Sigma \mathrm{Ca}\left(\mathrm{V}_{\mathrm{RGB}}-\mathrm{V}_{\mathrm{HB}}\right)
\end{array}
$$

where $\Sigma \mathrm{Ca}=0.5 \mathrm{EW}_{8498}+\mathrm{EW}_{8542}+0.6 \mathrm{EW}_{8662}, V_{R G B}$ is the magnitude (or, if using a composite spectrum, the average, S:N weighted magnitude) of the RGB star, and $V_{H B}$ is the mean $V$-magnitude of the horizontal branch (HB). Using $V_{H B}-V_{R G B}$ removes any strong dependence on distance or reddening in the calculated value of $[\mathrm{Fe} / \mathrm{H}]$, and gives the $\mathrm{Ca}$ II line strength at the level of the HB. For M31, we set this value to be $V_{H B}=25.17$ (Holland et al. 1996) 15. As the dSphs do not all sit at the same distance as M31, assuming this introduces a small error into our calculations, but it is at a far lower significance than the dominant uncertainty introduced by the noise within the spectra themselves. For individual stars, these measurements carry large uncertainties ( $\gtrsim 0.4$ dex), but these are significantly reduced when stacking the spectra into a composite in order to measure an average metallicity for a given population.

Uncertainties on the individual measurements of $[\mathrm{Fe} / \mathrm{H}]$ from our stellar spectra are typically large $(\geq$ $0.5 \mathrm{dex}$ ), so for a more robust determination of the average metallicities we co-add the spectra for each dSph (weighting by the $\mathrm{S}: \mathrm{N}$ of each individual stellar spectrum, which is required to be a minimum of $2.5 \AA^{-1}$ ) and measure the resulting EWs. In a few cases, not all $3 \mathrm{Ca}$ II lines are well resolved. For And V, IX, XVII, XVIII XXVI and XXVIII, the third Ca II line is significantly affected by skylines, whilst for And XXIV, the first $\mathrm{Ca}$ II line is distorted. In the case of And XIII, only the second $\mathrm{Ca}$ II line appears well resolved. In these cases, we neglect the affected lines in our estimate of $[\mathrm{Fe} / \mathrm{H}]$, and derive reduced equivalent widths

15 This assumed value is sensitive to age and metallicity effects, see Chen et al. 2009 for a discussion, however owing to the large distance of M31, small differences in this value within the M31 system will have a negligible effect on metallicity calculations from the unaffected lines. Where the third line is affected, this gives $\Sigma \mathrm{Ca}=1.5 \mathrm{EW}_{8498}+\mathrm{EW}_{8542}$. Where the second line is affected, we find $\Sigma \mathrm{Ca}=\mathrm{EW}_{8542}+\mathrm{EW}_{8662}$. Finally, where only the second line seems reliable we use $\Sigma \mathrm{Ca}=1.7 \mathrm{EW}_{8542}$. These coefficients are derived empirically from high $\mathrm{S}: \mathrm{N}$ spectra where the absolute values of $[\mathrm{Fe} / \mathrm{H}]$ are well known. We test these variations of $\Sigma \mathrm{Ca}$ by applying them to our high S:N co-added spectra where all three lines are well resolved, such as And XXI and XXV, and we find that all three formulae produce consistent values of $[\mathrm{Fe} / \mathrm{H}]$. The composite spectra for each satellite are shown in Figs. 21 and 22. In all cases, we find that our results are consistent with photometric metallicities derived in previous works.

In the MW, it has been observed that the average metallicities of the dSph population decrease with decreasing luminosity (e.g., Kirby et al. 2008, 2011). In Fig. 23. we plot the spectroscopic metallicities of the M31 dSphs (blue dSphs) as a function of absolute magnitude. We plot the MW dSphs as red triangles (Martin et al. 2007; Kirby et al. 2008, 2011; Belokurov et al. 2009; Koch et al. 2009). We also include those M31 dSphs for which only photometric measurements of $[\mathrm{Fe} / \mathrm{H}]$ are available (And I, II, III, VII and XIV, Kalirai et al. 2010; Tollerud et al. 2012), and these are highlighted as encircled blue points. The dashed line represents the best-fit to the MW dSph population from Kirby et al. (2011). The three relatively metal $\mathrm{rich}([\mathrm{Fe} / \mathrm{H}] \sim-1.5$ to -2.0$)$, faint $\left(L \sim 1000 \mathrm{~L}_{\odot}\right) \mathrm{MW}$ points are the three ultra faint dSphs Willman I, Boötes II and Segue 2, and these three were not included in the Kirby et al. (2011) analysis, where the best fit MW relation was determined. We see that the metallicities for a given luminosity in the M31 dSphs also loosely define a relationship of decreasing metallicity with decreasing luminosity, and they agree with that defined by their MW counterparts within their associated uncertainties. However, it is also noteworthy that for dSphs with $L<10^{6} \mathrm{~L}_{\odot}$, the Andromeda satellites are also consistent with having a constant metallicity of $\sim-1.8$. The same levelling off of average metallicity at lower luminosities was noted by McConnachie (2012), where they note that this break occurs at the same luminosity as a break in the luminosity-surface brightness relation for faint galaxies. As such, it could imply that the denisty of baryons in these systems, rather than the total number of baryons, could be the most important facor in determining their chemical evolution. The error bars we present here are still significant, so it is hard to fully interpret this result, but the hint of a metallicity floor in these lower luminosity systems is intriguing.

In Fig. [23, we highlight the positions of our three kinematic outliers, And XIX, XXI and XXV, and we see that they fall almost exactly on the MW relation. In this figure, systems that have experienced extreme tidal stripping would move horizontally to the left, as their luminosity would gradually decrease as stars are stripped, but their chemistry would remain unaffected. One would expect to see such behaviour only after the stellar component began to be removed in earnest, after the majority of the dark matter halo had been removed. If their central densities were lowered by some active feedback mechanism, such as SNII explosions (e.g., Zolotov et al. 2012), one would expect the objects to become more en- 

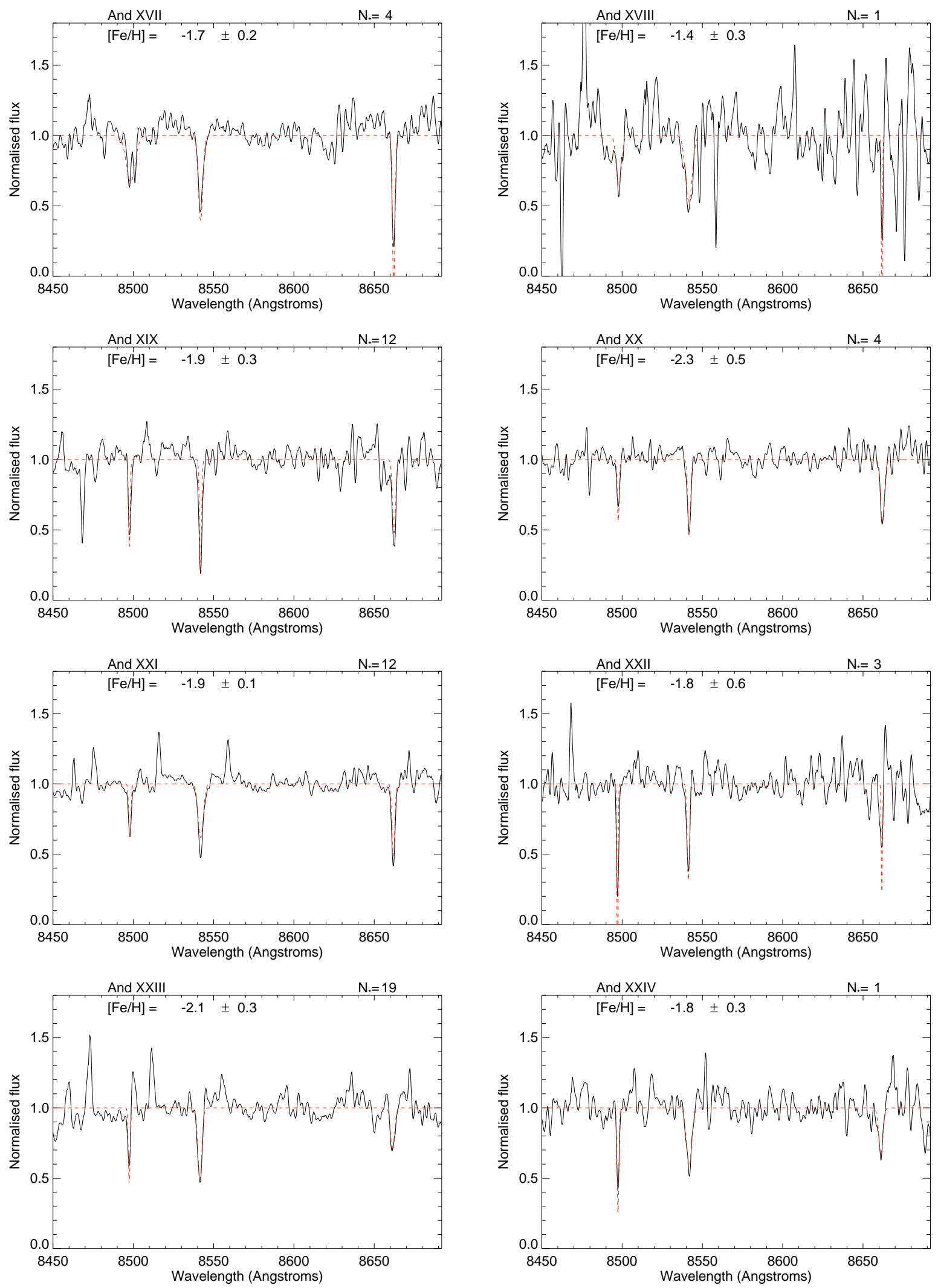

FIG. 21. - Figures of the S:N weighted composite spectra for each dSph. The average metallicity for each dSph, plus the associated uncertainty is printed on each panel (continued over page) 

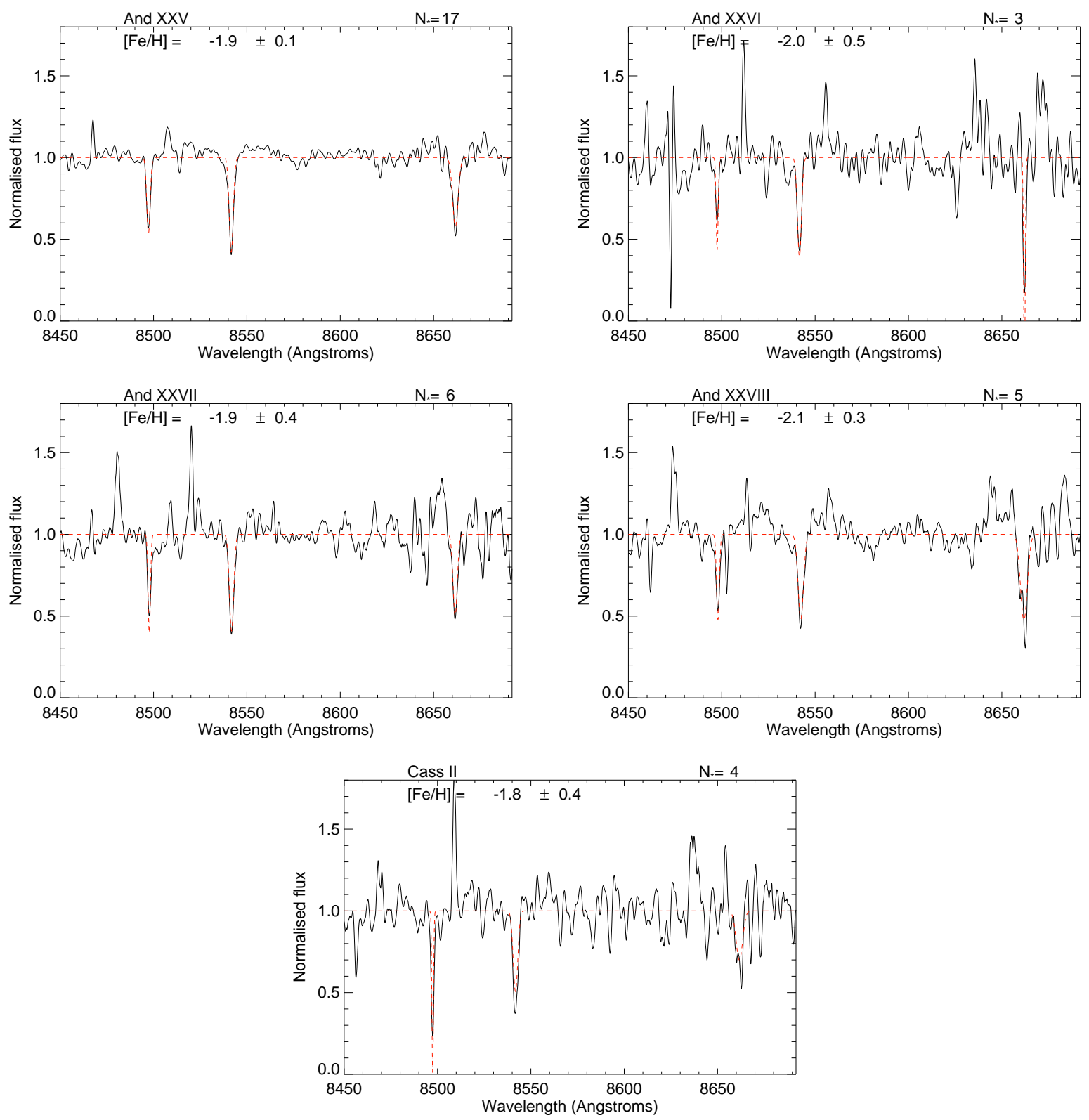

FIG. 22. - Figures of the S:N weighted composite spectra for each dSph (excluding And XV and And XVI). The average metallicity for each dSph, plus the associated uncertainty is printed on each panel (continued over page) 


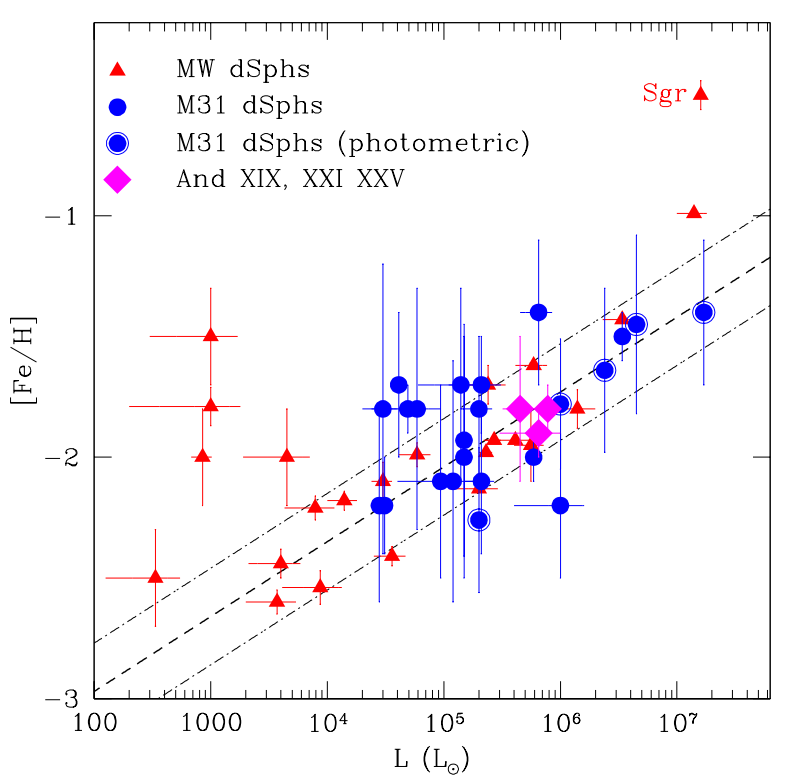

FIG. 23.- Spectroscopically derived $[\mathrm{Fe} / \mathrm{H}]$ vs. luminosity for all MW (red triangles, taken from Kirby et al.|2011, with additional measurements taken from Martin et al. 2007; Belokurov et al. 2009; Koch et al. 2009) and M31 dSphs (blue circles, this work). The solid line represents the best fit relationship between these two parameters as taken from Kirby et al. (2011). The dashed lines represent the $1 \sigma$ scatter about this relationship. We see that the M31 dSphs follow this relationship very well within their associated uncertainties. As we discuss in $\S 6$ those galaxies with $L<10^{6} \mathrm{~L}_{\odot}$ are also consistent with having a constant metallicity, which could indicate a metallicity floor in these fainter systems.

riched and perhaps brighter, moving them up and to the right, potentially allowing them to remain on the MW relation. To confirm that this was the case for And XIX, $\mathrm{XXI}$ and XXV, we would require more information on the abundances of these objects and their star formation histories, which we do not currently possess.

\section{CONCLUSIONS}

Using new and existing spectroscopic data from the Keck I LRIS and Keck II DEIMOS spectrographs, we have homogeneously derived kinematic properties for 18 of the 28 known Andromeda dSph galaxies. Using a combination of their $g-i$ colors, positions on the sky and radial velocities, we determine the likelihood of each observed star belonging to a given $\mathrm{dSph}$, thus filtering out MW foreground or M31 halo contaminants. We have measured both their systemic velocities and their velocity dispersions, with the latter allowing us to constrain the mass and densities within their half-light radii. For the first time, we confirm that And XVII, XIX, XX, XXIII, XXVI and Cass II are dark matter dominated objects, with dynamical mass-to-light ratios within the half-light radius of $[M / L]_{\text {half }}>10 \mathrm{M}_{\odot} / \mathrm{L}_{\odot}$.

For And XXV, a bright M31 dSph $\left(M_{V}=-9.7\right)$ we measure a mass-to-light ratio of only $[M / L]_{\text {half }}=$ $10.3_{-6.7}^{+7.0} \mathrm{M}_{\odot} / \mathrm{L}_{\odot}$ from a sample of 26 stars, meaning that it is consistent with a simple stellar system with no appreciable dark matter component within its $1 \sigma$ uncertainties. If this were confirmed with larger datasets, it would prove to be a very important object for our understanding of the formation and evolution of galaxies.
We compare our computed velocity dispersions and mass estimates with those measured for MW dSphs, and find that the majority of the M31 dSphs have very similar mass-size scalings to those of the MW. However, we note 3 significant outliers to these scalings, namely And XIX, XXI and XXV, who possess significantly lower velocity dispersions than expected for their size. These results builds on the identification of three potential outliers in the Tollerud et al. (2012) dataset (And XIV, XV and XVI). We suggest that the lower densities of the dark matter halos for these outliers could be an indication that they have encountered greater tidal stresses from their host over the course of their evolution, decreasing their masses. However, these bright systems still fall on the luminosity-metallicity relation established for the dSph galaxies of the Local Group. If these objects had undergone significant tidal disruption, we would expect them to lie above this relation. As such, this remains puzzling, and requires dedicated follow up studies to fully map out the kinematics of these unusual systems.

We measure the metallicities of all $18 \mathrm{dSphs}$ from their co-added spectra and find that they are consistent with the established MW trend of decreasing metallicity with decreasing luminosity.

This work represents a significant step forward in understanding the mass profiles of dwarf spheroidal galaxies. Far from residing in dark matter halos with identical mass profiles, we show that the halos of these objects are complex, and differ from one to the next, with their environment and tidal evolution imprinting themselves upon the dynamics of their stellar populations. The Andromeda system of dSphs presents us with an opportunity to better understand these processes, and our future work will further illuminate the evolutionary paths taken by these smallest of galaxies.

\section{ACKNOWLEDGMENTS}

We would like to thank Hans-Walter Rix for helpful discussions regarding this manuscript. We are also grateful to the referee for their helpful and detailed suggestions for improving this work. We thank the SPLASH collaboration for providing us with details of their observations of dSphs as presented in T12.

Most of the data presented herein were obtained at the W.M. Keck Observatory, which is operated as a scientific partnership among the California Institute of Technology, the University of California and the National Aeronautics and Space Administration. The Observatory was made possible by the generous financial support of the W.M. Keck Foundation.

Based in part on observations obtained with MegaPrime/MegaCam, a joint project of CFHT and CEA/DAPNIA, at the Canada-France-Hawaii Telescope (CFHT) which is operated by the National Research Council (NRC) of Canada, the Institute National des Sciences de l'Univers of the Centre National de la Recherche Scientifique of France, and the University of Hawaii.

Based in part on data collected at Subaru Telescope, which is operated by the National Astronomical Observatory of Japan.

The authors wish to recognize and acknowledge the very significant cultural role and reverence that the summit of Mauna Kea has always had within the indigenous Hawaiian community. We are most fortunate to have the 
TABLE 5

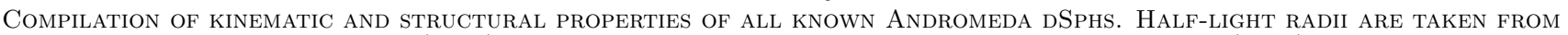
MCCONNACHIE (2012), AND UPDATED TO THE DistanCES PROVIDED IN CONN ET AL. (2012).

\begin{tabular}{|c|c|c|c|c|c|c|c|c|c|}
\hline Name & $M_{V}$ & $N_{*}^{(a)}$ & $\begin{array}{l}{[\mathrm{Fe} / \mathrm{H}]_{p h o t}} \\
(\mathrm{dex})\end{array}$ & $\begin{array}{c}{[\mathrm{Fe} / \mathrm{H}]_{\text {spec }}} \\
(\mathrm{dex})\end{array}$ & $\begin{array}{c}v_{r} \\
\left(\mathrm{~km} \mathrm{~s}^{-1}\right)\end{array}$ & $\begin{array}{c}\sigma_{v} \\
\left(\mathrm{~km} \mathrm{~s}^{-1}\right)\end{array}$ & $\begin{array}{r}r_{\text {half }} \\
\text { (pc) }\end{array}$ & $\begin{array}{c}\mathrm{D} \\
(\mathrm{kpc})\end{array}$ & Ref. \\
\hline And I & -11.8 & 80 & $-1.45 \pm 0.37$ & $\mathrm{~N} / \mathrm{A}$ & $-376.3 \pm 2.2$ & $10.2 \pm 1.9$ & $656_{-67}^{+68}$ & $727_{-17}^{+18}$ & $(1),(2),(3)$ \\
\hline And II & -12.6 & 95 & $-1.64 \pm 0.34$ & $\mathrm{~N} / \mathrm{A}$ & $-193.6 \pm 1.0$ & $7.3 \pm 0.8$ & $1136 \pm 46$ & $630 \pm 15$ & $(1),(3),(4)$ \\
\hline And III & -10.2 & 43 & $-1.78 \pm 0.27$ & $\mathrm{~N} / \mathrm{A}$ & $-344.3 \pm 1.7$ & $9.3 \pm 1.4$ & $463_{-45}^{+44}$ & $723_{-24}^{+18}$ & $(1),(2),(3)$ \\
\hline And V & -9.6 & 85 & $-1.6 \pm 0.3$ & $-1.8 \pm 0.2$ & $-397.3 \pm 1.5^{(b)}$ & $10.5 \pm 1.1^{(b)}$ & $302 \pm 44$ & $742_{-22}^{+24}$ & $(2),(3),(5)$ \\
\hline And VI & -11.5 & 38 & $-1.3 \pm 0.3$ & $-1.5 \pm 0.3$ & $-339.8 \pm 1.9$ & $12.4_{-1.3}^{+1.5}$ & $524 \pm 49$ & $783 \pm 28$ & $(3),(5),(6)$ \\
\hline And VII & -13.3 & 18 & $-1.4 \pm 0.3$ & $\mathrm{~N} / \mathrm{A}$ & $-307.2 \pm 1.3$ & $13.0 \pm 1.0$ & $776 \pm 42$ & $762 \pm 35$ & $(1),(2),(3)$ \\
\hline And IX & -8.1 & 32 & $-2.2 \pm 0.2$ & $-1.9 \pm 0.6$ & $-209.4 \pm 2.5^{(b)}$ & $10.9 \pm 2.0^{(b)}$ & $436_{-24}^{+68}$ & $600_{-23}^{+91}$ & $(1),(2),(3)$ \\
\hline And X & -8.1 & 22 & $-1.93 \pm 0.48$ & $\mathrm{~N} / \mathrm{A}$ & $-164.1 \pm 1.7$ & $6.4 \pm 1.4$ & $253_{-65}^{+24}$ & $670_{-39}^{+24}$ & $(1),(2),(3)$ \\
\hline And XI & -6.9 & 5 & $-2.0 \pm 0.2$ & $-2.0 \pm 0.3$ & $-427.5_{-3.5}^{+3.4}$ & $7.6_{-2.8}^{+4.0}$ & $158_{-23}^{+9.9}$ & $763_{-106}^{+29}$ & $(3),(6),(7)$ \\
\hline And XII & -6.4 & 8 & $-1.9 \pm 0.2$ & $-2.0 \pm 0.3$ & $-557.1 \pm 1.7$ & $0.0^{+4.0}$ & $324_{-72}^{+56}$ & $928_{-136}^{+40}$ & $(3),(6),(7)$ \\
\hline And XIII & -6.7 & 12 & $-2.0 \pm 0.2$ & $-1.9 \pm 0.7$ & $-185.4 \pm 2.4^{(b)}$ & $5.8 \pm 2.0^{(b)}$ & $172_{-39}^{+34}$ & $760_{-154}^{+126}$ & $(2),(3),(7)$ \\
\hline And XIV & -8.3 & 38 & $-2.26 \pm 0.3$ & $\mathrm{~N} / \mathrm{A}$ & $-480.6 \pm 1.2$ & $5.3 \pm 1.0$ & $392_{-205}^{+185}$ & $793_{-179}^{+23}$ & $(1),(2),(3)$ \\
\hline And XV & -9.4 & 29 & -1.1 & $\mathrm{~N} / \mathrm{A}$ & $-323 \pm 1.4^{(b)}$ & $4.0 \pm 1.4^{(b)}$ & $220_{-15}^{+29}$ & $626_{-35}^{+79}$ & $(1),(2),(3)$ \\
\hline And XVI & -9.4 & 7 & -1.7 & $-2.0 \pm 0.5$ & $-367.3 \pm 2.8^{(b)}$ & $3.8 \pm 2.9^{(b)}$ & $123_{-10}^{+13}$ & $476_{-29}^{+44}$ & $(1),(2),(3)$ \\
\hline And XVII & -8.5 & 7 & -1.9 & $-1.7 \pm 0.3$ & $-251.6_{-2.0}^{+1.8}$ & $2.9_{-1.9}^{+2.2}$ & $262_{-46}^{+10}$ & $727_{-25}^{+39}$ & $(1),(3),(6)$ \\
\hline And XVIII & -9.7 & 22 & $-1.8 \pm 0.5$ & $\mathrm{~N} / \mathrm{A}$ & $-332.1 \pm 2.7^{(b)}$ & $9.7 \pm 2.3^{(b)}$ & $325 \pm 24$ & $1214_{-43}^{+45}$ & $(1),(2),(3)$ \\
\hline And XIX & -9.3 & 27 & $-1.9 \pm 0.4$ & $-1.9 \pm 0.6$ & $-111.6_{-1.4}^{+1.6}$ & $4.7_{-1.4}^{+1.6}$ & $1481_{-268}^{+62}$ & $821_{-148}^{+32}$ & $(1),(3),(6)$ \\
\hline And XX & -6.3 & 4 & $-1.5 \pm 0.5$ & $-2.3 \pm 0.8$ & $\begin{array}{r}-456.2_{-3.6}^{+3.4} \\
-3\end{array}$ & $7.1_{-2.5}^{+3.4}$ & $114_{-12}^{+31}$ & $741_{-52}^{+48}$ & $(1),(3),(6)$ \\
\hline And XXI & -9.9 & 32 & -1.8 & $-1.8 \pm 0.4$ & $-362.5 \pm 0.9$ & $4.5_{-1.0}^{+1.5}$ & $842 \pm 77$ & $827_{-25}^{+23}$ & $(1),(3),(6)$ \\
\hline And XXII & -6.5 & 12 & -1.8 & $-1.85 \pm 0.1$ & $-129.8 \pm 2.0$ & $2.8_{-1.4}^{+1.0}$ & $252_{-47}^{+28}$ & $\begin{array}{c}-25 \\
920_{-139}^{+32}\end{array}$ & $(1),(2),(3),(8)$ \\
\hline And XXIII & -10.2 & 42 & $-1.8 \pm 0.2$ & $-2.3 \pm 0.7$ & $-237.7 \pm 1.2$ & $7.1 \pm 1.0$ & $1001_{-52}^{+53}$ & $748_{-21}^{+31}$ & $(1),(3),(6)$ \\
\hline AndXXIV & -7.6 & 3 & $-1.8 \pm 0.2$ & $-1.8 \pm 0.3$ & $-128.2 \pm 5.2^{(c)}$ & $0.0^{+7.3(c)}$ & $548_{-37}^{+31}$ & $898_{42}^{+28}$ & $(1),(3),(6)$ \\
\hline And XXV & -9.7 & 25 & $-1.8 \pm 0.2$ & $-2.1 \pm 0.2$ & $-107.8 \pm 1.0$ & $3.0_{-1.1}^{+1.2}$ & $642_{-74}^{-37}$ & $736_{-69}^{+23}$ & $(1),(3),(6)$ \\
\hline And XXVI & -7.1 & 6 & $-1.9 \pm 0.2$ & $-1.8 \pm 0.5$ & $-261.6_{-2.8}^{+3.0}$ & $8.6_{-2.2}^{+2.8}$ & $219_{-52}^{+67}$ & $754_{-164}^{+218}$ & $(1),(3),(6)$ \\
\hline AndXXVII & -7.9 & 11 & $-1.7 \pm 0.2$ & $-1.5 \pm 0.28$ & $-539.6_{-4.5}^{+4.8}$ & $14.8_{-3.1}^{+4.3}$ & $657_{-271}^{+112}$ & $1255_{-474}^{+424}$ & $(1),(3),(6)$ \\
\hline AndXXVIII & -8.5 & 17 & $-2.0 \pm 0.2$ & $-2.1 \pm 0.3$ & $\begin{array}{l}-4.5 \\
-326.2 \pm 2.7\end{array}$ & $6.6_{-2.1}^{+2.9}$ & $210_{-50}^{+60}$ & $650_{-80}^{+150}$ & $(6),(9)$ \\
\hline AndXXIX & -8.3 & 24 & $-1.8 \pm 0.2$ & $\mathrm{~N} / \mathrm{A}$ & $-194.4 \pm 1.5$ & $5.7 \pm 1.2$ & $360 \pm 60$ & $730 \pm 75$ & $(10),(11)$ \\
\hline And XXX (Cass II) & -8.0 & 8 & $-1.6 \pm 0.4$ & $-2.2 \pm 0.4$ & $-139.8_{-6.6}^{+6.0}$ & $11.8_{-4.7}^{+7.7}$ & $267_{-36}^{+23}$ & $681_{-78}^{+32}$ & $(3),(6),(12)$ \\
\hline
\end{tabular}

Note. — ${ }^{(a)}$ Number of spectroscopically confirmed member ${ }^{(b)}$ Kinematics taken from T12 rather than this work, as they possess greater numbers of member stars. stars. (c) Owing to shorter than typical exposure time, lower resolution data and difficult observing conditions, this kinematic identification of And XXIV remains tentative, and needs to be confirmed with further follow-up. References: (1) McConnachie (2012), (2) Tollerud et al. (2012), (3) Conn et al. (2012), (4) Kalirai et al. (2010) (5) Collins et al. (2011), (6) This work, (7) Collins et al. (2010), (8) Chapman et al. (2012), (9) Slater et al. (2011), (10) Tollerud et al. (2013), (11) Bell et al. (2011), (12) Irwin et al. (in prep).

opportunity to conduct observations from this mountain. Funding for SDSS-III has been provided by the Alfred P. Sloan Foundation, the Participating Institutions, the National Science Foundation, and the U.S. Department of Energy Office of Science. The SDSS-III web site is http://www.sdss3.org/.

SDSS-III is managed by the Astrophysical Research Consortium for the Participating Institutions of the SDSS-III Collaboration including the University of Arizona, the Brazilian Participation Group, Brookhaven National Laboratory, University of Cambridge, Carnegie Mellon University, University of Florida, the French Participation Group, the German Participation Group, Harvard University, the Instituto de Astrofisica de Canarias, the Michigan State/Notre Dame/JINA Participation Group, Johns Hopkins University, Lawrence Berkeley National Laboratory, Max Planck Institute for As- trophysics, Max Planck Institute for Extraterrestrial Physics, New Mexico State University, New York University, Ohio State University, Pennsylvania State University, University of Portsmouth, Princeton University, the Spanish Participation Group, University of Tokyo, University of Utah, Vanderbilt University, University of Virginia, University of Washington, and Yale University.

R.I. gratefully acknowledges support from the Agence Nationale de la Recherche though the grant POMMME (ANR 09-BLAN-0228).

G.F.L. gratefully acknowledges financial support for his ARC Future Fellowship (FT100100268) and through the award of an ARC Discovery Project (DP110100678).

N.B. gratefully acknowledges financial support through the award of an ARC Discovery Project (DP110100678).

A.K. thanks the Deutsche Forschungsgemeinschaft for funding from Emmy-Noether grant Ko 4161/1.

\section{REFERENCES}


Amorisco, N. C. \& Evans, N. W. 2012, MNRAS, 419, 184

Battaglia, G., Irwin, M., Tolstoy, E., Hill, V., Helmi, A., Letarte, B., \& Jablonka, P. 2008, MNRAS, 383, 183

Bell, E. F., Slater, C. T., \& Martin, N. F. 2011, ApJ, 742, L15

Belokurov, V., Walker, M. G., Evans, N. W., Gilmore, G., Irwin, M. J., Mateo, M., Mayer, L., Olszewski, E., Bechtold, J., \& Pickering, T. 2009, MNRAS, 397, 1748

Blais-Ouellette, S., Amram, P., \& Carignan, C. 2001, AJ, 121, 1952

Brasseur, C. M., Martin, N. F., Macciò, A. V., Rix, H.-W., \& Kang, X. 2011a, ApJ, 743, 179

Brasseur, C. M., Martin, N. F., Rix, H.-W., Irwin, M., Ferguson, A. M. N., McConnachie, A. W., \& de Jong, J. 2011b, ApJ, 729, 23

Brooks, A. M. \& Zolotov, A. 2012, ArXiv e-prints

Bullock, J. S., Kravtsov, A. V., \& Weinberg, D. H. 2000, ApJ, 539,517

Bullock, J. S., Stewart, K. R., Kaplinghat, M., Tollerud, E. J., \& Wolf, J. 2010, ApJ, 717, 1043

Chapman, S. C., Ibata, R., Irwin, M., Koch, A., Letarte, B., Martin, N., Collins, M., Lewis, G. F., McConnachie, A., Peñarrubia, J., Rich, R. M., Trethewey, D., Ferguson, A., Huxor, A., \& Tanvir, N. 2008, MNRAS, 390, 1437

Chapman, S. C., Ibata, R., Lewis, G. F., Ferguson, A. M. N., Irwin, M., McConnachie, A., \& Tanvir, N. 2005, ApJ, 632, L87 —. 2006, ApJ, 653, 255

Chapman, S. C., Peñarrubia, J., Ibata, R., McConnachie, A., Martin, N., Irwin, M., Blain, A., Lewis, G. F., Letarte, B., Lo, K., Ludlow, A., \& O'neil, K. 2007, ApJ, 662, L79

Chapman, S. C., Widrow, L., Collins, M. L. M., Dubinski, J., Ibata, R. A., Rich, M., Ferguson, A. M. N., Irwin, M. J., Lewis, G. F., Martin, N., McConnachie, A., Penarrubia, J., \& Tanvir, N. 2012, ArXiv e-prints

Chen, Y. Q., Zhao, G., \& Zhao, J. K. 2009, ApJ, 702, 1336

Collins, M. L. M., Chapman, S. C., Irwin, M. J., Martin, N. F., Ibata, R. A., Zucker, D. B., Blain, A., Ferguson, A. M. N., Lewis, G. F., McConnachie, A. W., \& Peñarrubia, J. 2010, MNRAS, 407, 2411

Collins, M. L. M., Chapman, S. C., Rich, R. M., Irwin, M. J., Peñarrubia, J., Ibata, R. A., Arimoto, N., Brooks, A. M., Ferguson, A. M. N., Lewis, G. F., McConnachie, A. W., \& Venn, K. 2011, MNRAS, 417, 1170

Conn, A. R., Ibata, R. A., Lewis, G. F., Parker, Q. A., Zucker, D. B., Martin, N. F., McConnachie, A. W., Irwin, M. J., Tanvir, N., Fardal, M. A., Ferguson, A. M. N., Chapman, S. C., \& Valls-Gabaud, D. 2012, ApJ, 758, 11

Conn, A. R., Lewis, G. F., Ibata, R. A., Parker, Q. A., Zucker, D. B., McConnachie, A. W., Martin, N. F., Irwin, M. J., Tanvir, N., Fardal, M. A., \& Ferguson, A. M. N. 2011, ApJ, 740, 69

Côté, P., Mateo, M., Olszewski, E. W., \& Cook, K. H. 1999, ApJ, 526,147

de Blok, W. J. G. 2005, ApJ, 634, 227

de Blok, W. J. G. \& Bosma, A. 2002, A\&A, 385, 816

de Blok, W. J. G., Bosma, A., \& McGaugh, S. 2003, MNRAS, 340, 657

Dotter, A., Chaboyer, B., Jevremović, D., Kostov, V., Baron, E., \& Ferguson, J. W. 2008, ApJS, 178, 89

Ferguson, A. M. N., Irwin, M. J., Ibata, R. A., Lewis, G. F., \& Tanvir, N. R. 2002, AJ, 124, 1452

Garrison-Kimmel, S., Rocha, M., Boylan-Kolchin, M., Bullock, J., \& Lally, J. 2013, ArXiv e-prints

Holland, S., Fahlman, G. G., \& Richer, H. B. 1996, AJ, 112, 1035

Ibata, R., Chapman, S., Ferguson, A. M. N., Lewis, G., Irwin, M., \& Tanvir, N. 2005, ApJ, 634, 287

Ibata, R., Irwin, M., Lewis, G., Ferguson, A. M. N., \& Tanvir, N. 2001, Nature, 412, 49

Ibata, R., Martin, N. F., Irwin, M., Chapman, S., Ferguson, A. M. N., Lewis, G. F., \& McConnachie, A. W. 2007, ApJ, 671, 1591

Ibata, R., Sollima, A., Nipoti, C., Bellazzini, M., Chapman, S. C., \& Dalessandro, E. 2011, ApJ, 738, 186

Ibata, R. A., Lewis, G. F., Conn, A. R., Irwin, M. J., McConnachie, A. W., Chapman, S. C., Collins, M. L., Fardal, M., Ferguson, A. M. N., Ibata, N. G., Mackey, A. D., Martin, N. F., Navarro, J., Rich, R. M., Valls-Gabaud, D., \& Widrow, L. M. 2013, Nature, 493, 62

Illingworth, G. 1976, ApJ, 204, 73
Irwin, M. J., Ferguson, A. M. N., Huxor, A. P., Tanvir, N. R., Ibata, R. A., \& Lewis, G. F. 2008, ApJ, 676, L17

Irwin, M. J., Ferguson, A. M. N., Ibata, R. A., Lewis, G. F., \& Tanvir, N. R. 2005, ApJ, 628, L105

Jardel, J. R. \& Gebhardt, K. 2012, ApJ, 746, 89

Kalirai, J. S., Beaton, R. L., Geha, M. C., Gilbert, K. M., Guhathakurta, P., Kirby, E. N., Majewski, S. R., Ostheimer, J. C., Patterson, R. J., \& Wolf, J. 2010, ApJ, 711, 671

Kassin, S. A., de Jong, R. S., \& Pogge, R. W. 2006, ApJS, 162, 80

Kazantzidis, S., Łokas, E. L., Callegari, S., Mayer, L., \& Moustakas, L. A. 2011, ApJ, 726, 98

Kirby, E. N., Lanfranchi, G. A., Simon, J. D., Cohen, J. G., \& Guhathakurta, P. 2011, ApJ, 727, 78

Kirby, E. N., Simon, J. D., Geha, M., Guhathakurta, P., \& Frebel, A. 2008, ApJ, 685, L43

Klypin, A., Kravtsov, A. V., Valenzuela, O., \& Prada, F. 1999, ApJ, 522, 82

Koch, A., Wilkinson, M. I., Kleyna, J. T., Irwin, M., Zucker, D. B., Belokurov, V., Gilmore, G. F., Fellhauer, M., \& Evans, N. W. 2009, ApJ, 690, 453

Koposov, S., Belokurov, V., Evans, N. W., Hewett, P. C., Irwin, M. J., Gilmore, G., Zucker, D. B., Rix, H.-W., Fellhauer, M., Bell, E. F., \& Glushkova, E. V. 2008, ApJ, 686, 279

Koposov, S. E., Gilmore, G., Walker, M. G., Belokurov, V., Wyn Evans, N., Fellhauer, M., Gieren, W., Geisler, D., Monaco, L., Norris, J. E., Okamoto, S., Penarrubia, J., Wilkinson, M., Wyse, R. F. G., \& Zucker, D. B. 2011, ArXiv e-prints, 1105.4102

Koposov, S. E., Yoo, J., Rix, H.-W., Weinberg, D. H., Macciò, A. V., \& Escudé, J. M. 2009, ApJ, 696, 2179

Kravtsov, A. 2010, Advances in Astronomy, 2010

Letarte, B., Chapman, S. C., Collins, M., Ibata, R. A., Irwin, M. J., Ferguson, A. M. N., Lewis, G. F., Martin, N., McConnachie, A., \& Tanvir, N. 2009, MNRAS, 400, 1472

Martin, N. F., Ibata, R. A., Chapman, S. C., Irwin, M., \& Lewis, G. F. 2007, MNRAS, 380, 281

Martin, N. F., Ibata, R. A., Irwin, M. J., Chapman, S., Lewis, G. F., Ferguson, A. M. N., Tanvir, N., \& McConnachie, A. W. 2006, MNRAS, 371, 1983

Martin, N. F. \& Jin, S. 2010, ApJ, 721, 1333

Martin, N. F., McConnachie, A. W., Irwin, M., Widrow, L. M., Ferguson, A. M. N., Ibata, R. A., Dubinski, J., Babul, A., Chapman, S., Fardal, M., Lewis, G. F., Navarro, J., \& Rich, R. M. 2009, ApJ, 705, 758

Mateo, M. L. 1998, ARA\&A, 36, 435

McConnachie, A. W. 2012, AJ, 144, 4

McConnachie, A. W., Arimoto, N., \& Irwin, M. 2007, MNRAS, 379,379

McConnachie, A. W., Huxor, A., Martin, N. F., Irwin, M. J., Chapman, S. C., Fahlman, G., Ferguson, A. M. N., Ibata, R. A., Lewis, G. F., Richer, H., \& Tanvir, N. R. 2008, ApJ, 688, 1009

McConnachie, A. W. \& Irwin, M. J. 2006a, MNRAS, 365, 1263

- 2006b, MNRAS, 365, 902

McConnachie, A. W. et al. 2009, Nature, 461, 66

Moore, B., Ghigna, S., Governato, F., Lake, G., Quinn, T., Stadel, J., \& Tozzi, P. 1999, ApJ, 524, L19

Navarro, J. F., Frenk, C. S., \& White, S. D. M. 1997, ApJ, 490, 493

Nickerson, S., Stinson, G., Couchman, H. M. P., Bailin, J., \& Wadsley, J. 2011, MNRAS, 415, 257

Niederste-Ostholt, M., Belokurov, V., Evans, N. W., Gilmore, G., Wyse, R. F. G., \& Norris, J. E. 2009, MNRAS, 398, 1771

Peñarrubia, J., Benson, A. J., Walker, M. G., Gilmore, G., McConnachie, A. W., \& Mayer, L. 2010, MNRAS, 406, 1290

Peñarrubia, J., McConnachie, A. W., \& Navarro, J. F. 2008a, ApJ, 672, 904

Peñarrubia, J., Navarro, J. F., \& McConnachie, A. W. 2008b, ApJ, 673, 226

Plummer, H. C. 1911, MNRAS, 71, 460

Pontzen, A. \& Governato, F. 2012, MNRAS, 421, 3464

Richardson, J. C., Irwin, M. J., McConnachie, A. W., Martin, N. F., Dotter, A. L., Ferguson, A. M. N., Ibata, R. A., Chapman, S. C., Lewis, G. F., Tanvir, N. R., \& Rich, R. M. 2011, ApJ, 732, 76

Richstone, D. O. \& Tremaine, S. 1986, AJ, 92, 72 
Schlegel, D. J., Finkbeiner, D. P., \& Davis, M. 1998, ApJ, 500, 525

Ségall, M., Ibata, R. A., Irwin, M. J., Martin, N. F., \& Chapman, S. 2007, MNRAS, 375, 831

Simon, J. D. \& Geha, M. 2007, ApJ, 670, 313

Simon, J. D., Geha, M., Minor, Q. E., Martinez, G. D., Kirby, E. N., Bullock, J. S., Kaplinghat, M., Strigari, L. E., Willman, B., Choi, P. I., Tollerud, E. J., \& Wolf, J. 2011, ApJ, 733, 46

Slater, C. T., Bell, E. F., \& Martin, N. F. 2011, ApJ, 742, L14

Sohn, S. T., Majewski, S. R., Muñoz, R. R., Kunkel, W. E., Johnston, K. V., Ostheimer, J. C., Guhathakurta, P., Patterson, R. J., Siegel, M. H., \& Cooper, M. C. 2007, ApJ, 663,960

Somerville, R. S. 2002, ApJ, 572, L23

Spano, M., Marcelin, M., Amram, P., Carignan, C., Epinat, B., \& Hernandez, O. 2008, MNRAS, 383, 297

Starkenburg, E., Hill, V., Tolstoy, E., González Hernández, J. I., Irwin, M., Helmi, A., Battaglia, G., Jablonka, P., Tafelmeyer, M., Shetrone, M., Venn, K., \& de Boer, T. 2010, A\&A, 513, A34+

Strigari, L. E., Bullock, J. S., Kaplinghat, M., Simon, J. D., Geha, M., Willman, B., \& Walker, M. G. 2008, Nature, 454, 1096

Swaters, R. A., Verheijen, M. A. W., Bershady, M. A., \& Andersen, D. R. 2003, ApJ, 587, L19
Tollerud, E. J., Bullock, J. S., Strigari, L. E., \& Willman, B. 2008, ApJ, 688, 277

Tollerud, E. J., Beaton, R. L., Geha, M. C., Bullock, J. S., Guhathakurta, P., Kalirai, J. S., Majewski, S. R., Kirby, E. N., Gilbert, K. M., Yniguez, B., Patterson, R. J., Ostheimer, J. C., Cooke, J., Dorman, C. E., Choudhury, A., \& Cooper, M. C. 2012, ApJ, 752, 45

Tollerud, E. J., Geha, M. C., Vargas, L. C., \& Bullock, J. S. 2013, ArXiv e-prints

Walker, M. G., Belokurov, V., Evans, N. W., Irwin, M. J., Mateo, M., Olszewski, E. W., \& Gilmore, G. 2009a, ApJ, 694, L144

Walker, M. G., Mateo, M., Olszewski, E. W., Gnedin, O. Y., Wang, X., Sen, B., \& Woodroofe, M. 2007, ApJ, 667, L53

Walker, M. G., Mateo, M., Olszewski, E. W., Peñarrubia, J., Wyn Evans, N., \& Gilmore, G. 2009b, ApJ, 704, 1274

Walker, M. G. \& Peñarrubia, J. 2011, ApJ, 742, 20

Walsh, S. M., Willman, B., \& Jerjen, H. 2009, AJ, 137, 450

Wolf, J., Martinez, G. D., Bullock, J. S., Kaplinghat, M., Geha, M., Muñoz, R. R., Simon, J. D., \& Avedo, F. F. 2010, MNRAS, 406, 1220

Zolotov, A., Brooks, A. M., Willman, B., Governato, F., Pontzen, A., Christensen, C., Dekel, A., Quinn, T., Shen, S., \& Wadsley, J. 2012, ArXiv e-prints

Zucker, D. B. et al. 2004, ApJ, 612, L121

-. 2007, ApJ, 659, L21

\section{APPENDIX}

\section{A. TESTING THE MEMBERShiP PROBABILITY ALGORITHM}

\section{A.1. The inclusion of a velocity term in the calculation of $P_{i}$}

In T12, the authors do not impose a velocity probability criterion for their membership calculations. Instead they require all member stars to have a total probability, based on their positions and colors, of $P_{\text {member }}>0.1$, and then apply a $3 \sigma$ clipping to this final sample to prevent any outliers from significantly inflating their calculated velocity dispersions. In our analysis, we have avoided making any hard cuts to our sample by also utilising prior information on the velocities of our expected contaminant populations and member stars. In $\S 3.3$, we tested our velocity probability criteria was not overly biasing our final measurements of velocity dispersion to artificially lower values with the introduction of an extra parameter, $\eta$, that allows us to add additional weight to stars in the tails of the Gaussian velocity distribution. However, we can further test our velocity criterion by removing it entirely from the probabilistic determination, and instead implementing the same cuts presented by T12. This involves cutting stars where $P_{i}<0.1$ (as determined from $P_{C M D}$ and $P_{\text {dist }}$ ), and also by iteratively removing all stars that have velocities that do not lie within $3 \sigma$ of the mean of the remaining sample. In Table A1, we present the results of this on our measured values of $v_{r}$ and $\sigma_{v}$ for our full sample of dSphs. For all objects (bar And XXVII, which is a unique case, as described in $\S 4.11$ ) the systemic velocities derived are within $\sim 2-3 \mathrm{~km} \mathrm{~s}^{-1}$ of one another. The velocity dispersions we measure from our full algorithm tend to be slightly higher on average, and this is to be expected as we do not cut any stars from our analysis, and therefore outliers in the velocity profile may be assigned non-negligible membership probabilities that will allow them to increase this measurement. By and large, these differences are not significant, with the final values agreeing to well within their $1 \sigma$ uncertainties.

It is interesting that our algorithm appears to perform better when dealing with dSphs where the number of member stars is low. This is best demonstrated by And XI (see Fig. A1). Our algorithm identifies 5 stars with non-negligible probabilities of membership, clustered around $v_{r} \sim-430 \mathrm{~km} \mathrm{~s}^{-1}$. Our full algorithm measures a systemic velocity of $v_{r}=-427.5_{-3.4}^{+3.5} \mathrm{~km} \mathrm{~s}^{-1}$ and a velocity dispersion of $\sigma_{v}=7.5_{-2.8}^{+4.0} \mathrm{~km} \mathrm{~s}^{-1}$. One of these stars is slightly offset from the other 4 with a more negative velocity of $v_{r}=-456.8 \mathrm{~km} \mathrm{~s}^{-1}$. Although this star has a reasonably high probability of being a member based on its distance from the centre of And XI, and its position in the CMD, it does not survive the $3 \sigma$ velocity clipping procedure of T12. As the number of member stars is so low, cutting one star from the sample can have a significant effect, and as such, while the T12 procedure determines a very similar systemic velocity of $v_{r}=-425.0 \pm 3.1 \mathrm{~km} \mathrm{~s}^{-1}$ it is unable to resolve a velocity dispersion. This effect is also seen other systems (such as And XIII, XVII, XXII and XXVI), although it is typically less pronounced.

Another regime where our algorithm performs better than that of T12 is where the systemic velocity of the system in question is within the regime of the contaminating Milky Way K-dwarfs. An example of this is the unusual system, And XIX, where our algorithm measures a systemic velocity of $v_{r}=-111.6_{-1.4}^{+1.6} \mathrm{~km} \mathrm{~s}^{-1}$ and a velocity dispersion of $\sigma_{v}=4.7_{-1.4}^{+1.6}$. However, the procedure of T12 is less able to resolve the kinematics of the system, measuring $v_{r}=-109.3 \pm 5.3 \mathrm{~km} \mathrm{~s}^{-1}$ and a velocity dispersion of $\sigma_{v}=1.8_{-1.8}^{+9.1}$. The much larger uncertainty on the dispersion is a result of including Milky Way contaminants in the sample which can be difficult to cut out without applying prior knowledge of the velocity profile of this population. $3 \sigma$ clipping allows outliers to contribute more significantly to the measured profile in this instance, increasing the uncertainty. A similar effect is seen in the And XXIV and And XXX (Cass II) objects, which also have systemic velocities in the Milky Way contamination regime. 

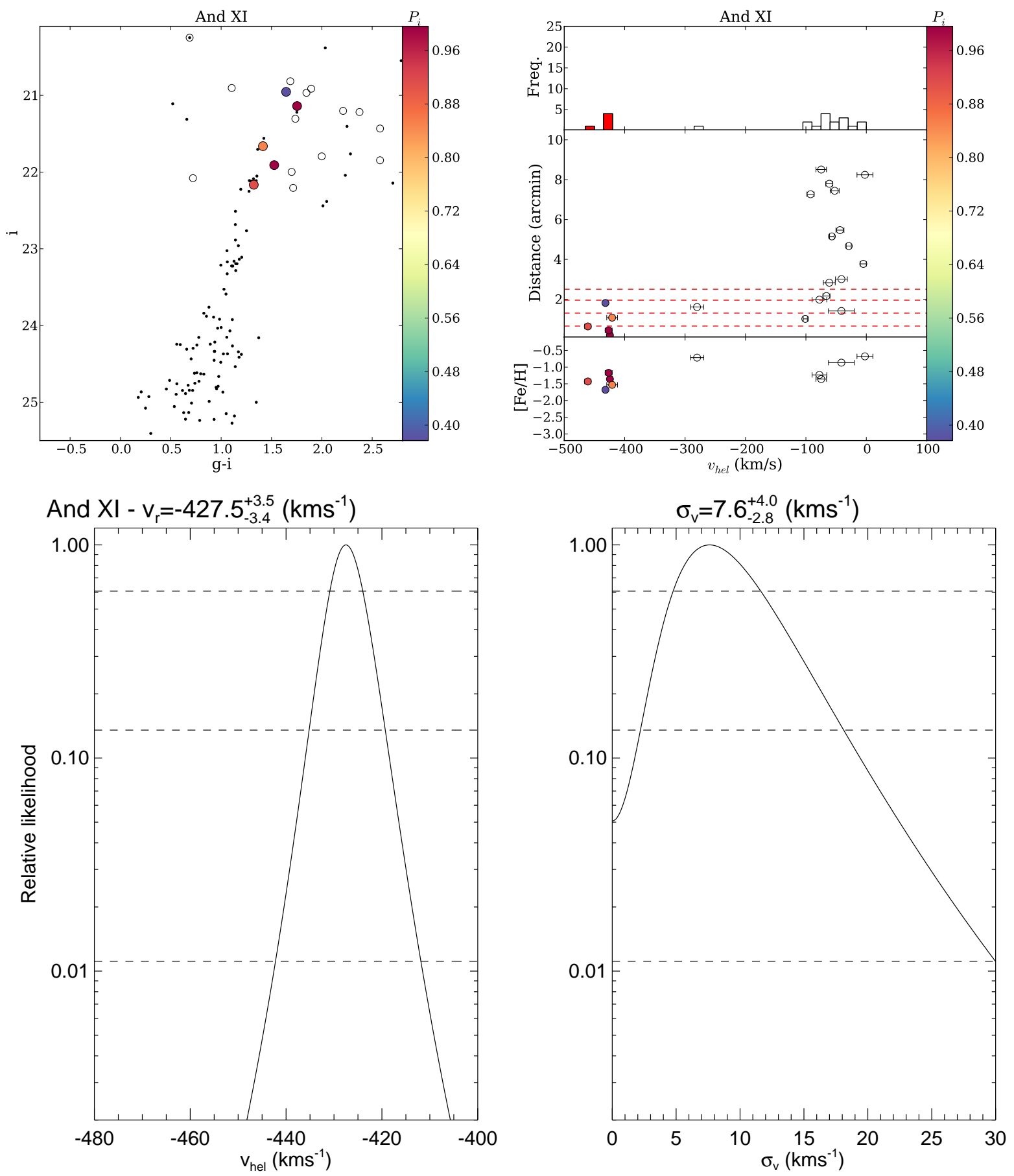

FIg. A1. - As Fig. 6 but for And XI. Using our full probabilistic algorithm (including our $P_{v e l}$ term), 5 stars are identified as likely members. When implementing the $3 \sigma$ clipping of T12, the star with $v_{r} \sim-450 \mathrm{~km} \mathrm{~s}^{-1}$ is removed from the analysis, despite having a high probability of membership based on its color $\left(P_{C M D}\right)$ and distance from the centre of the dSph $\left(P_{d i s t}\right)$. This has a substantial effect of the calculated velocity dispersion, as shown in Table A1

These results lead us to conclude that the inclusion of a $P_{v e l}$ term in our analysis allows us to more effectively determine the true kinematics of the systems we are studying. Further, as no cuts to the sample are required using this method, it allows for a more unbiased study of the kinematics of dSphs than that of T12. 
TABLE A1

Measured Velocities $\left(v_{r}\right)$ AND Dispersions $\left(\sigma_{v}\right)$ FOR OuR Full SAMPle of DSPHS AS CALCULATED USING CUTS ON PROBABILITY OF MEMBERSHIP CALCULATED FROM ONLY COLOR AND POSITION OF STARS, COMBINED WITH $3 \sigma$ CLIPPING ON VELOCITIES VS. THOSE CALCULATED USING OUR FULL PROBABILISTIC ALGORITHM.

\begin{tabular}{lcccc}
\hline \hline \multicolumn{1}{c}{ Object } & $\begin{array}{c}v \& P_{i} \text { cuts } \\
v_{r}\left(\mathrm{~km} \mathrm{~s}^{-1}\right)\end{array}$ & $\sigma_{v}\left(\mathrm{~km} \mathrm{~s}^{-1}\right)$ & $\begin{array}{c}\text { Full algorithm } \\
v_{r}\left(\mathrm{~km} \mathrm{~s}^{-1}\right)\end{array}$ & $\sigma_{v}\left(\mathrm{~km} \mathrm{~s}^{-1}\right)$ \\
\hline And V & $-391.1 \pm 2.9$ & $10.8_{-2.3}^{+3.0}$ & $-391.5 \pm 2.7$ & $12.2_{-1.9}^{+2.5}$ \\
And VI & $-339.0 \pm 3.0$ & $11.9_{-2.3}^{+2.9}$ & $-339.8 \pm 1.8$ & $12.4_{-1.3}^{+1.5}$ \\
And XI & $-425.0 \pm 3.1$ & $0^{+3.5}$ & $-427.5_{-3.4}^{+3.5}$ & $7.6_{-2.8}^{+4.0}$ \\
And XII & $-558.8 \pm 3.7$ & $0^{+6.8}$ & $-557.1 \pm 1.7$ & $0^{+4.0}$ \\
And XIII & $-203.8 \pm 8.4$ & $0^{+16.2}$ & $-204.8 \pm 4.9$ & $0.0^{+8.1}$ \\
And XVII & $-260.0_{-7.8}^{+8.0}$ & $1.8_{-1.8}^{+9.1}$ & $-254.3_{-3.7}^{+3.3}$ & $2.9_{-2.9}^{+5.0}$ \\
And XVIII & $-345.1 \pm 3.3$ & $0^{+4.4}$ & $-346.8 \pm 2.0$ & $0^{+2.7}$ \\
And XIX & $-109.3 \pm 5.3$ & $1.5_{-1.5}^{+6.8}$ & $-111.6_{-1.4}^{+1.6}$ & $4.7_{-1.4}^{+1.6}$ \\
And XX & $-454.6_{-5.7}^{+4.6}$ & $7.7_{-3.9}^{+8.4}$ & $-456.2_{-3.6}^{+3.1}$ & $7.1_{-2.5}^{+3.9}$ \\
And XXI & $-363.4_{-1.8}^{+2.0}$ & $3.2_{-2.1}^{+2.3}$ & $-362.5 \pm 0.9$ & $4.5_{-1.0}^{+1.2}$ \\
And XXII & $-131.4 \pm 2.7$ & $0^{+3.1}$ & $-129.8 \pm 2.0$ & $2.8_{-1.4}^{+1.9}$ \\
And XXIII & $-236.9 \pm 2.1$ & $8.4_{-1.5}^{+1.9}$ & $-237.7 \pm 1.2$ & $7.1 \pm 1.0$ \\
And XXIV & $-129.2 \pm 3.6$ & $0^{+6.1}$ & $-129.9_{-4.4}^{+4.3}$ & $3.5_{-3.5}^{+6.6}$ \\
And XXV & $-107.7_{-1.8}^{+1.9}$ & $3.3_{-1.8}^{+2.2}$ & $-107.8 \pm 1.0$ & $3.0_{-1.1}^{+1.2}$ \\
And XXVI & $-264.1 \pm 4.5$ & $0^{+4.8}$ & $-261.7_{-2.8}^{+3.1}$ & $8.7_{-2.3}^{+2.9}$ \\
And XXVII & $-517.6_{-43.2}^{+42.8}$ & $19.3_{-19}^{+17}$ & $-539.6_{-4.5}^{+4.7}$ & $14.8_{-3.1}^{+4.3}$ \\
And XXX (CassII) & $-140.1_{-9.3}^{+8.6}$ & $14.1_{-6.1}^{+12.9}$ & $-139.8_{-6.6}^{+6.0}$ & $11.8_{-4.7}^{+7.7}$ \\
& & & & \\
\hline
\end{tabular}

TABLE A2

The EFFECT OF INCLUDING LOW S:N DATA IN OUR ANALYSIS ON DETERMINING $v_{r}$ AND $\sigma_{v}$.

\begin{tabular}{|c|c|c|c|c|c|c|c|c|c|}
\hline Object & $N_{*}$ & $\begin{array}{c}\text { And XXI } \\
v_{r}\left(\mathrm{~km} \mathrm{~s}^{-1}\right)\end{array}$ & $\sigma_{v}\left(\mathrm{~km} \mathrm{~s}^{-1}\right)$ & $N_{*}$ & $\begin{array}{l}\text { And XXIII } \\
v_{r}\left(\mathrm{~km} \mathrm{~s}^{-1}\right)\end{array}$ & $\sigma_{v}\left(\mathrm{~km} \mathrm{~s}^{-1}\right)$ & $N_{*}$ & $\begin{array}{c}\text { And XXV } \\
v_{r}\left(\mathrm{~km} \mathrm{~s}^{-1}\right)\end{array}$ & $\sigma_{v}\left(\mathrm{~km} \mathrm{~s}^{-1}\right)$ \\
\hline $\mathrm{S}: \mathrm{N}>2$ & 20 & $-362.9 \pm 0.9$ & $3.5_{-0.7}^{+0.9}$ & 22 & $-238.0 \pm 1.2$ & $6.6 \pm 1.1$ & 27 & $-107.7 \pm 0.9$ & $2.7 \pm 1.1$ \\
\hline $\mathrm{S}: \mathrm{N}>3$ & 11 & $-364.2 \pm 0.9$ & $3.1 \pm 0.8$ & 10 & $-238.3 \pm 1.4$ & $5.1_{-1.2}^{+1.4}$ & 24 & $-107.7 \pm 1.0$ & $3.0 \pm 1.2$ \\
\hline $\mathrm{S}: \mathrm{N}>4$ & 5 & $-363.9 \pm 1.5$ & $4.0_{-1.1}^{+1.5}$ & 5 & $-239.4 \pm 1.1$ & $5.7_{-1.3}^{+1.5}$ & 16 & $-108.2 \pm 1.2$ & $3.0_{-1.2}^{+1.4}$ \\
\hline $\mathrm{S}: \mathrm{N}>5$ & 3 & $-362.6_{-2.3}^{+2.2}$ & $4.5_{-1.5}^{+2.1 .6}$ & 2 & $-239.5 \pm 1.9$ & $0.0^{-4.9}$ & 13 & $-109.0 \pm 1.2$ & $2.8_{-1.3}^{+1.2}$ \\
\hline
\end{tabular}

NoTE. - $\mathrm{xxx}$

\section{A.2. The effect of low signal-to-noise data on measuring $v_{r}$ and $\sigma_{v}$}

For our brightest targets $(i \lesssim 22.5)$ the S:N of our spectra is typically $>3 \AA^{-1}$. However, as our targets become fainter, so too their $\mathrm{S}: \mathrm{N}$ falls. For spectra with $\mathrm{S}: \mathrm{N} \gtrsim 1.5 \AA^{-1}$, our pipeline is still able to measure velocities based on the Ca II triplet, with reasonable measurement uncertainties. However, it is prudent to check whether the inclusion of these velocities, calculated from significantly noisier spectra, has a detrimental effect on our ability to measure the kinematic properties of our dSph sample.

Such a test is straightforward to implement. We have a number of dSphs within our sample for which our probabilistic analysis identifies 30 likely members (such as And XXI, XXIII and XXV). We can therefore use these samples to impose S:N cuts on our data to see the effect of this on our measurements of $v_{r}$ and $\sigma_{v}$. We present the results of this test in Table A2, and our finding is that, as the level of our imposed S:N cut increases (and so the number of included stars decreases), the systemic velocity remains more or less constant. The measured velocity dispersion, however, shows some variation. In the case of And XXI and XXV, the dispersion increases with increased S:N, however not significantly. In both cases the dispersion calculated from the higher S:N data lies well within $1 \sigma$ of that calculated from the lower $\mathrm{S}: \mathrm{N}$ data. Intuitively, this makes sense as the spectra with higher S:N are likely to have lower velocity uncertainties, and so our maximum-likelihood analysis will attribute more of the spread in measured velocities to an intrinsic dispersion, rather than to our measurement errors. In the case of And XXIII, we find the opposite to be true. As our $\mathrm{S}: \mathrm{N}$ cut increases, we find that our measured dispersion decreases. This may be because the number of member stars in subsequent quality cuts drops off more rapidly for And XXIII than And XXI and XXV. This suggests that we should be extra cautious when interpreting our measured velocity dispersions for dSphs where both the average S:N of member stars, and the number of member stars, is low. 
TABLE A3

THE EFFECT OF VARYING SAMPLE SIZE $\left(N_{*}\right)$ ON THE MEASUREMENTS OF SYSTEMIC VELOCITY, $v_{r}$, AND

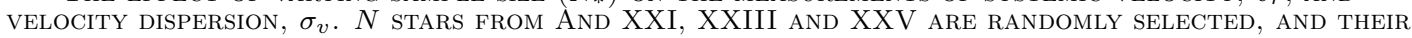
PROPERTIES ARE DERIVED USING OUR FULL PROBABILISTIC ANALYSIS. THIS WAS REPEATED 1000 TIMES. VALUES REPORTED BELOW ARE THE AVERAGES FROM 1000 REALIZATIONS, WITH THE UNCERTAINTIES REPRESENT THE STANDARD DEVIATION OF THE 1000 REALIZATIONS.

\begin{tabular}{ccccccc}
\hline \hline Object & And XXI & & And XXIII & \multicolumn{3}{c}{ And XXV } \\
$N_{*}$ & $v_{r}\left(\mathrm{~km} \mathrm{~s}^{-1}\right)$ & $\sigma_{v}\left(\mathrm{~km} \mathrm{~s}^{-1}\right)$ & $v_{r}\left(\mathrm{~km} \mathrm{~s}^{-1}\right)$ & $\sigma_{v}\left(\mathrm{~km} \mathrm{~s}^{-1}\right)$ & $v_{r}\left(\mathrm{~km} \mathrm{~s}^{-1}\right)$ & $\sigma_{v}\left(\mathrm{~km} \mathrm{~s}^{-1}\right)$ \\
\hline 4 & $-362.6 \pm 3.1$ & $5.0 \pm 3.3$ & $-237.2 \pm 3.8$ & $7.5 \pm 3.6$ & $-108.8 \pm 2.4$ & $5.4 \pm 4.3$ \\
6 & $-363.0 \pm 2.0$ & $4.0 \pm 1.9$ & $-237.3 \pm 3.0$ & $6.9 \pm 2.6$ & $-108.0 \pm 2.7$ & $4.0 \pm 2.3$ \\
8 & $-362.9 \pm 2.1$ & $4.2 \pm 2.0$ & $-236.8 \pm 2.8$ & $6.9 \pm 2.3$ & $-107.6 \pm 2.0$ & $2.7 \pm 1.1$ \\
10 & $-362.7 \pm 1.8$ & $4.1 \pm 1.8$ & $-237.3 \pm 2.5$ & $6.5 \pm 2.1$ & $-107.9 \pm 1.5$ & $3.1 \pm 1.0$ \\
15 & $-362.9 \pm 1.5$ & $4.3 \pm 1.2$ & $-237.4 \pm 2.0$ & $6.8 \pm 1.4$ & $-107.9 \pm 1.5$ & $3.1 \pm 1.0$ \\
20 & $-362.9 \pm 1.3$ & $4.3 \pm 1.2$ & $-237.6 \pm 1.7$ & $6.9 \pm 1.4$ & $-107.8 \pm 1.2$ & $3.0 \pm 0.9$ \\
25 & $-363.0 \pm 1.0$ & $4.4 \pm 1.0$ & $-237.4 \pm 1.4$ & $7.1 \pm 1.7$ & $-107.8 \pm 1.0$ & $3.1 \pm 0.8$ \\
30 & $-362.8 \pm 1.1$ & $4.4 \pm 1.1$ & $-237.7 \pm 1.3$ & $7.2 \pm 1.4$ & - & - \\
Full sample & $\mathbf{- 3 6 2 . 5} \pm \mathbf{0 . 9}$ & $\mathbf{4 . 5}+\mathbf{1 . 2}$ & $\mathbf{- 2 3 7 . 7} \pm \mathbf{1 . 2}$ & $\mathbf{7 . 1} \pm \mathbf{1 . 0}$ & $\mathbf{- 1 0 7 . 8} \pm \mathbf{1 . 0}$ & $\mathbf{3 . 0}+\mathbf{1 . 1}$ \\
& & & & & & \\
\hline
\end{tabular}

\section{A.3. The effect of small sample sizes on determining kinematic properties of dwarf galaxies}

Obtaining reliable velocities for member stars of faint and distant systems is a difficult task that can only be achieved with the largest optical telescopes, such as Keck. Given the demand for facilities such as this, any observing time awarded must be used as effectively as possible, and this often means compromising between deep pointings for a few objects, and shallower pointings for a number of objects. With longer or multiple exposures on a single target, one can build up impressive samples of member stars for an individual dSph. For example, the SPLASH collaboration observed a total of 95 members in And II, one of the brightest M31 dSph companions (Kalirai et al. 2010) by taking 2 separate exposure fields over this large object. However, multiple exposures such as these produce diminishing returns as you move down the luminosity scale to fainter, more compact dSphs. This is both because of their smaller size with respect to the DEIMOS field of view, and the fewer number of bright stars available on the RGB to target. In this case, the only way to identify more members is by integrating for longer, but given the paucity of stars, the trade-off between time spent exposing and additional members observed can be quite expensive. Such difficulties inevitably lead to the inference of dynamical properties for an entire system from a handful of stars. It is important for us to understand the effect this bias has on our results, and how reliable the quoted values are. We test this using our datasets for which we identify $>25$ member stars, namely And XIX, XXI, XXIII and XXV using the following method. We select $4,6,8,10,15,20,25$ and 30 stars at random from each dataset and then measure the systemic velocity and velocity dispersion using our probability algorithm. This was repeated 1000 times for each sample size. In cases where the algorithm is unable to resolve a velocity dispersion, we throw out the result and resimulate, as null results here will affect our averages and will not inform us whether the instances in which we are able to resolve a velocity dispersion from small numbers of stars are producing valid, reliable result. We display the resulting values in Table $\mathrm{A3}$, with the true value recovered from the full sample shown in bold in the final row for comparison. We show that in all these cases, the systemic velocity and velocity dispersion are recovered well within the scatter of the 1000 simulations even when dealing with sample sizes as small as 4 stars, so long as the measurement is resolved. In cases where we are unable to resolve a dispersion, we find that our resulting uncertainties are not meaningful. This is shown explicitly in the case of And XVIII, where we can compare our upper limit for the velocity dispersion as determined from our algorithm with the dispersion calculated in T12 from a much larger dataset. We see that our uncertainty is not consistent with their result. As such, we advise that in all cases where we calculate velocity dispersions from small samples $\left(N_{*}<8\right.$, And XI, XII, XX XXIV and XXVI), the dispersion measurements should be treated as indications of the likely dispersion, and need to be confirmed with follow-up studies.

\section{A.4. Testing our algorithm on the SPLASH sample of M31 dSphs}

In T12, the authors reported on the kinematic properties of 15 M31 dSphs, And I, III, V, VII, IX, X, XI, XII, XIII, XIV, XV, XVI, XVIII, XXI and XXII, and the positions, and measured velocities (plus uncertainties) for each likely member stars were published as part of that work. The authors were kind enough to also give us access to these properties for their non-member stars so that we might run our algorithm over the full samples to see if we reproduce their results. Our technique for assigning membership probability differs from theirs in that we use the velocities of stars as an additional criterion for membership, whereas they use a cut on both the resulting probability $(P$ (member $>0.1)$, and a $3 \sigma$ clipping on the velocity. In addition, where we use PAndAS CFHT MegaCam $g-$ and $i$-band photometry for our membership analysis, the SPLASH team use their own Washington-DDO51 filter photometric dataset, in membership classification. As such, small differences might be expected, but if our technique is robust our results should well mirror those of T12. In Table A4 we compare our calculated values of $v_{r}$ and $\sigma_{v}$ to those published in T12. As the measurements made in T12 for And XI and XII are made from only 2 stars, we do not include these in this test. In general, the results from both analyses agree to within $1 \sigma$ of one another, with the majority of them being well within this bound. Typically, we find that our procedure measures slightly larger values for $\sigma_{v}$ than that of T12 (with the exception of And IX, And XVIII and And XXII). This is to be expected, as we do 
TABLE A4

\begin{tabular}{lcccc}
\hline \hline \multicolumn{1}{c}{ Object } & $\begin{array}{c}\text { T12 analysis } \\
v_{r}\left(\mathrm{~km} \mathrm{~s}^{-1}\right)\end{array}$ & $\sigma_{v}\left(\mathrm{~km} \mathrm{~s}^{-1}\right)$ & $\begin{array}{c}\text { Our analysis } \\
v_{r}\left(\mathrm{~km} \mathrm{~s}^{-1}\right)\end{array}$ & $\sigma_{v}\left(\mathrm{~km} \mathrm{~s}^{-1}\right)$ \\
\hline And I & $-376.3 \pm 2.2$ & $10.2 \pm 1.9$ & $-376.3 \pm 1.3$ & $10.6 \pm 1.0$ \\
And III & $-344.3 \pm 1.7$ & $9.3 \pm 1.4$ & $-344.2 \pm 1.2$ & $10.1 \pm 1.9$ \\
And V & $-397.3 \pm 1.5$ & $10.5 \pm 1.1$ & $-396.0 \pm 1.0$ & $11.4 \pm 1.2$ \\
And VII & $-307.2 \pm 1.3$ & $13.0 \pm 1.0$ & $-307.1 \pm 1.1$ & $13.1 \pm 0.9$ \\
And IX & $-209.4 \pm 2.5$ & $10.9 \pm 2.0$ & $-210.3 \pm 1.9$ & $10.2_{-1.7}^{+1.9}$ \\
And X & $-164.1 \pm 1.7$ & $6.4 \pm 1.4$ & $-165.3 \pm 1.5$ & $6.0_{-1.2}^{+1.3}$ \\
And XIII & $-185.4 \pm 2.4$ & $5.8 \pm 2.0$ & $-183.0_{-2.3}^{+2.4}$ & $8.6_{-1.7}^{+2.1}$ \\
And XV & $-323 \pm 1.4$ & $4.0 \pm 1.4$ & $-322.6 \pm 1.1$ & $6.0_{-1.8}^{+2.0}$ \\
And XVI & $-367.3 \pm 2.8$ & $3.8 \pm 2.9$ & $-366.1_{-3.1}^{+4.0}$ & $4.2_{-4.2}^{+4.8}$ \\
And XVIII & $-332.1 \pm 2.7$ & $9.7 \pm 2.3$ & $-330.7_{-4.1}^{+3.9}$ & $7.5_{-3.1}^{+4.5}$ \\
And XXI & $-361.4 \pm 5.8$ & $7.2 \pm 5.5$ & $-358.9_{-5.6}^{+5.1}$ & $8.5_{-5.1}^{+6.3}$ \\
And XXII & $-126.8 \pm 3.1$ & $3.54_{-2.49}^{+4.16}$ & $-124.2_{-4.5}^{+4.6}$ & $0.0^{+5.7}$ \\
& & & &
\end{tabular}

not cut stars from our analysis based on their velocity, instead we down-weight their probability of membership. As such, those stars considered as outliers would naturally inflate our dispersions above those measured by T12, but the effect is marginal. These results demonstrate that our technique for assigning probability of membership of individual stars within M31 dSphs based on their photometric properties and velocities is robust, and comparable to that of T12. However, as discussed in $\S$ A.1, we find that our technique is superior as it requires no cuts to the final dataset to be made, reducing the bias in these measurements.

\section{B. REANALYZING OUR PREVIOUSLY PUBLISHED RESULTS}

To ensure our analysis of the global properties of the M31 dSph population in $\S 5.1$ is homogenous, we reanalyzed our previously published datasets using our new probability algorithm. Here we briefly summarize the results of this analysis and compare the new results to the published works. The objects we discuss here are And V, VI (first published in Collins et al. 2011), XI, XII and XIII (published in Chapman et al. 2005, 2007; Collins et al. 2010). And $\mathrm{V}$ was observed with the LRIS instrument, while the remaining objects were observed with the DEIMOS instrument.

\section{B.1. Andromeda $V$}

Andromeda V (And V) was observed using the LRIS instrument on Keck I rather than the DEIMOS instrument on Keck II. LRIS has a lower resolution than DEIMOS, and a smaller field of view, which lowers the accuracy of velocity measurement and limits us to only $\sim 50$ targets within a mask compared with $100-200$ for a DEIMOS mask. The raw data were also not reduced using our standard pipeline, owing to problems with the arc-lamp calibrations, and were instead analyzed using the NOAO.ONEDSPEC and NOAO.TWODSPEC packages in IRAF. The results from this reduction, plus an analysis of the data using hard cuts in velocity, distance and color to determine membership were first published in Collins et al. (2011). Our full probabilistic analysis identifies 17 stars with a non-negligible probability of belonging to And V. Our technique determines a systemic velocity of $v_{r}=-391.5 \pm 2.7 \mathrm{~km} \mathrm{~s}^{-1}$ and $\sigma_{v}=12.2_{-1.9}^{+2.5} \mathrm{~km} \mathrm{~s}^{-1}$. Comparing these values to our previously published results $\left(v_{r}=-393.1 \pm 4.2 \mathrm{~km} \mathrm{~s}^{-1}\right.$ and $\sigma_{v}=11.5_{-4.3}^{+5.3} \mathrm{~km} \mathrm{~s}^{-1}$, Collins et al. 2011) we find them to be consistent within the quoted uncertainties. We also compare our results to those of T12, who measured $v_{r}=397.3 \pm 1.5 \mathrm{~km} \mathrm{~s}^{-1}$ and $\sigma_{v}=10.5 \pm 1.1 \mathrm{~km} \mathrm{~s}^{-1}$ from a larger sample of stars (85 members cf. 17) using the higher resolution DEIMOS spectrograph. The velocity dispersions of both are consistent within their $1 \sigma$ uncertainties, as are the systemic velocities. Given the difference of a factor of 5 in number of probable member stars between our study and that of T12, this consistency is reassuring, and demonstrates the ability of our technique to accurately determine the kinematics of M31 dSph galaxies from small sample sizes.

\section{B.2. Andromeda VI}

As And VI sits at a large projected distance from the centre of M31 $(\sim 270 \mathrm{kpc})$, it was not observed as part of the PAndAS survey and we were unable to use CFHT data for our $P_{C M D}$ determination. Instead, we use Subaru Suprime-cam data (PI. N. Arimoto, see Collins et al. 2011 for a full discussion of this data). Using our full probabilistic analysis, we identify 45 stars with $P_{i}>10^{-6}$. Our technique determines a most-likely $v_{r}=-339.8 \pm 1.8 \mathrm{~km} \mathrm{~s}^{-1}$, with $\sigma_{v}=12.4_{-1.3}^{+1.5} \mathrm{~km} \mathrm{~s}^{-1}$. Comparing these values with the results of Collins et al. (2011) who measured $v_{r}=$ $-344.8 \pm 2.5 \mathrm{~km} \mathrm{~s}^{-1}$ and $\sigma_{v}=9.4_{-2.4}^{+3.2} \mathrm{~km} \mathrm{~s}^{-1}$, we see that both the systemic velocities and the velocity dispersions are consistent within quoted uncertainties. The slight differences between our previous study and this work are simply a result of the application of our new technique.

\section{B.3. Andromeda XI}

The kinematic properties for Andromeda XI as measured from this DEIMOS data set were first published in Collins et al. (2010). Here we identify 5 stars as probable members. We determine most-likely parameters of 
$v_{r}=-427.5_{-3.4}^{+3.5} \mathrm{~km} \mathrm{~s}^{-1}$ and $\sigma_{v}=7.6_{-2.8}^{+4.0} \mathrm{~km} \mathrm{~s}^{-1}$. In Collins et al. (2010) we measured $v_{r}=-419.4_{-3.8}^{+4.4} \mathrm{~km} \mathrm{~s}^{-1}$ and we were unable to resolve the velocity dispersion for the dSph, measuring $\sigma_{v}=0.0^{+4.6} \mathrm{~km} \mathrm{~s}^{-1}$ (where the upper bound represents the formal $1 \sigma$ uncertainty on the unresolved dispersion), which implied a higher systemic velocity and lower velocity dispersion for And XI. However, in that analysis, one star with a velocity of $\sim-440 \mathrm{~km} \mathrm{~s}^{-1}$ was considered to be an outlier based on its velocity, and thus excluded from the kinematic analysis. Here, our algorithm gives this star a non-zero probability of membership, which likely decreases the systemic velocity and increases the dispersion. T12 also presented observations for And XI, but they were not able to cleanly detect the galaxy. They identified 2 stars with highly negative velocities $\left(\sim-460 \mathrm{~km} \mathrm{~s}^{-1}\right)$, which are offset from our systemic velocity by $\sim 30 \mathrm{~km} \mathrm{~s}^{-1}$. Given the very negative velocities of their 2 stars, the probability of them both being M31 contaminants seems low, and some other explanation may be more suitable. Between observations, there is one star common to both $(\alpha=00: 46: 19.10, \delta=+33: 48: 4.1)$, for which we measure a velocity of $-427.16 \mathrm{~km} \mathrm{~s}^{-1}$ compared with $-461.6 \mathrm{~km} \mathrm{~s}^{-1}$. This amounts to a statistical difference at the level of $5 \sigma$. One obvious avenue to check is that there has been no velocity offset introduced by a rogue skyline that falls within the region of one of the three Ca II lines. We have carefully checked the spectra of each of our 5 probable members to see if this has been the case. We also rederive the velocity based on cross-correlations with each of the three lines individually, rather than with the full triplet. We find these results, and their average to be entirely consistent within the associated errors from the velocities derived using the technique discussed in $\S 2.2$. This large discrepancy is puzzling, particularly as the methods used to measure velocities in this work are almost identical to those of T12. Without further data it is not possible for either team to pin down the exact issue, or which of the datasets gives the true systemic velocity. This argues for taking further observations within these faint systems, in the hopes of better understanding both the systems themselves, and any systematics introduced by the DEIMOS instrument. It is comforting that, in all cases where this offset is observed, the velocity dispersions measured by each team are consistent with one another, suggesting the problem affects all observations identically.

In this case, we have identified a greater number of potential members stars than in the work of T12, the spectra for all of which have relatively high S:N (S:N $\left.\sim 5-10 \AA^{-1}\right)$. Therefore, our results should be considered as more robust than those of $\mathrm{T} 12$.

\section{B.4. Andromeda XII}

Kinematic properties for Andromeda XII as determined from the DEIMOS data set presented here were previously published in Chapman et al. (2007) and Collins et al. (2010), and in both cases, membership was largely determined using hard cuts in velocity. This object has been of particular interest as it possesses an extremely negative systemic velocity with respect to Andromeda, suggesting that it is on its first infall into the local group (see Chapman et al. 2007 for a full discussion). Using our new algorithm, we measure $v_{r}=-557.1 \mathrm{~km} \mathrm{~s}^{-1}$ and an unresolved velocity dispersion of $\sigma_{v}=0.0^{+4.0} \mathrm{~km} \mathrm{~s}^{-1}$, where the upper bound represents the formal $1 \sigma$ uncertainty on the measurement. As the dispersion is unresolved, the lower error bound is undefined, as having a negative velocity dispersion is unphysical. These values are completely consistent with the results of Chapman et al. (2007) and Collins et al. (2010) $\left(v_{r}=\right.$ $-558.4 \pm 3.2 \mathrm{~km} \mathrm{~s}^{-1}$ and $\left.\sigma_{v}=2.6_{-2.6}^{+5.1} \mathrm{~km} \mathrm{~s}^{-1}\right)$. T12 also presented observations for And XII, but as for And XI, they were not able to cleanly detect the galaxy. They identified 2 stars with highly negative velocities $\left(\sim-530 \mathrm{~km} \mathrm{~s}^{-1}\right)$, which are offset from our systemic velocity by $\sim 30 \mathrm{~km} \mathrm{~s}^{-1}$. In this instance, both the stars they observed overlap with two of our likely members, situated at $\alpha=00: 47: 28.63, \delta=+34: 22: 43.1$ and $\alpha=00: 47: 24.69, \delta=+34: 22: 23.9$, and these velocities are offset from those that we measure at a statistical level of 3.8 $\sigma$. This suggests that the self-same calibration effect that causes an offset between our results for And XI and those of T12, is present here also. In the case of And XII, our mask was observed over two separate nights, giving us two velocity measurements for each star (as discussed in Chapman et al. 2007 and Collins et al. 2010), and we saw no evidence for systematic offsets of this magnitude in the night to night velocities, making a calibration error within our dataset seem unlikely, though not impossible. We therefore conclude that, owing to our larger sample of members and repeat observations, our measurements for the kinematic properties of And XII are more robust than those of T12.

\section{B.5. Andromeda XIII}

The kinematic properties of Andromeda XIII were also presented in Collins et al. (2010), And XIII sits at a large projected distance from Andromeda ( $\sim 120 \mathrm{kpc}$ ) in the southern M31 halo, so we expect contamination from the Milky Way and Andromeda halo to be low. It is surprising then, that we see significant structure within our DEIMOS field. This is also seen within the 3 fields observed by T12, who attribute this over-density of stars located at $v_{r} \sim-120 \mathrm{~km} \mathrm{~s}^{-1}$ to an association with the TriAnd over-density within the Galactic halo. We too see a number of stars between $-140 \mathrm{~km} \mathrm{~s}^{-1}$ and $-100 \mathrm{~km} \mathrm{~s}^{-1}$. From their positions within the CMD of And XIII, they appear more consistent with MW foreground K-dwarfs than M31 RGB stars. As such, these are also likely associated to this MW substructure.

Using our full probabilistic analysis, we identify the most probable And XIII stars as those 4 that cluster around $v \sim-200 \mathrm{~km} \mathrm{~s}^{-1}$, and we determine $v_{r}=-204.8 \pm 4.9 \mathrm{~km} \mathrm{~s}^{-1}$ and are unable to resolve a velocity dispersion, with $\sigma_{v}=0.0^{+8.1}$, where the upper limit indicates the formal $1 \sigma$ uncertainty on the likelihood distribution. Given the very large uncertainties on these values (mostly a factor of the low number of member stars) it comes as no surprise perhaps that these results are consistent with the results in Collins et al. $(2010)\left(v_{r}=-195.0_{-8.4}^{+7.4} \mathrm{~km} \mathrm{~s}^{-1}\right.$ and $\left.\sigma_{v}=9.7_{-4.5}^{+8.9}\right)$, 
although not with those of T12 $\left(v_{r}=-185.4 \pm 2.4 \mathrm{~km} \mathrm{~s}^{-1}\right.$ and $\left.\sigma_{v}=5.6 \pm 2.0 \mathrm{~km} \mathrm{~s}^{-1}\right)$, where they derived parameters from three times the number of member stars that we present here. Given the highly substructured nature of the And XIII field, and the fact that our detection is at a very low significance (only 4 stars), we find that the T12 measurements for the kinematics of And XIII are more robust than ours. 